Ecole d'Eté Systèmes Optiques

\title{
Radiométrie et détection optique
}

\author{
J.L. Meyzonnette
}

Institut d'Optique Theorique et Appliqué, E.S.O., Centre Universitaire d'Orsay, Bat. 503, BP. 147, F-91403 Orsay cedex, France

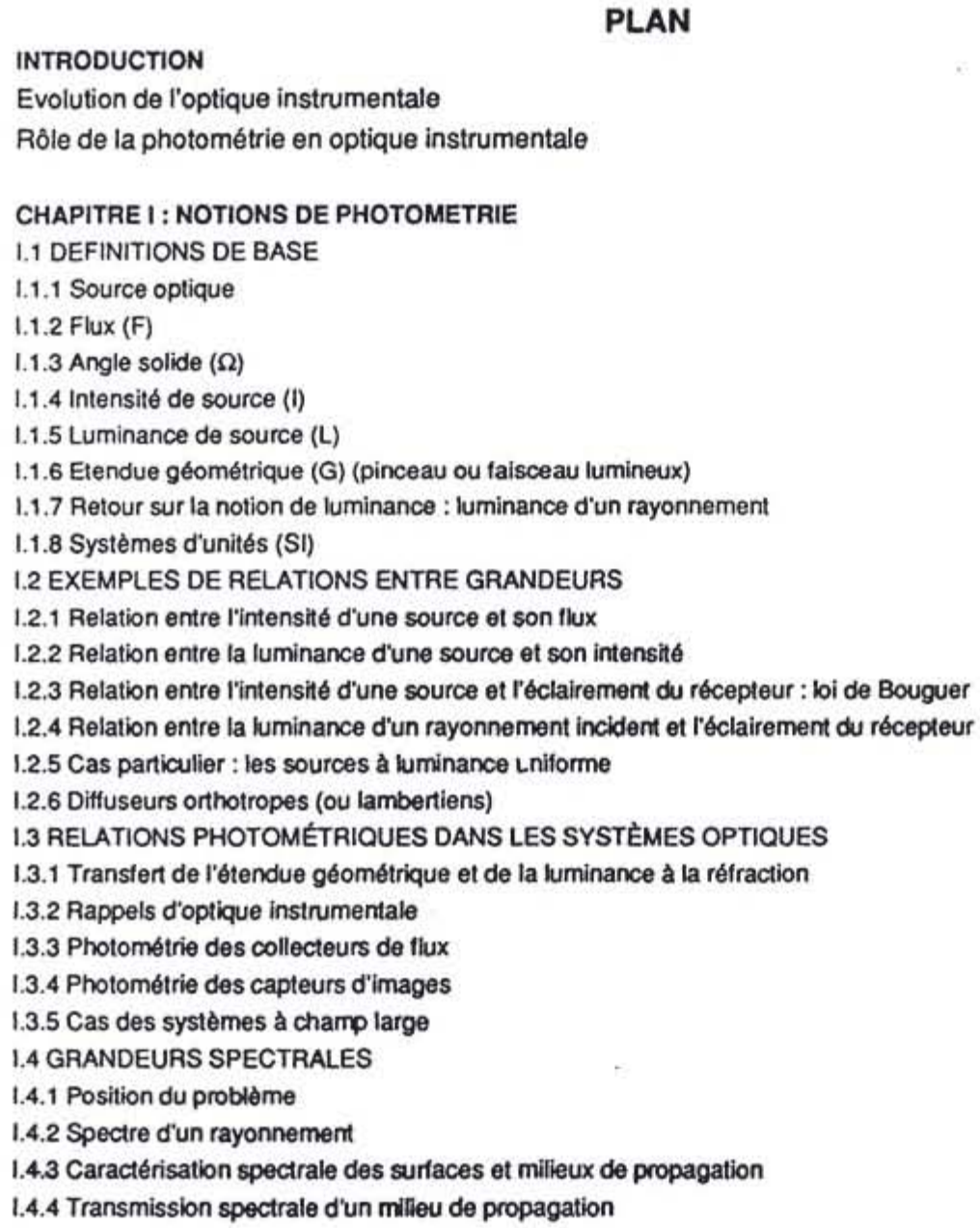

PLAN

INTRODUCTION

Evolution de l'optique instrumentale

Rôle de la photométrie en optique instrumentale

CHAPITRE I : NOTIONS DE PHOTOMETRIE

I.1 DEFINITIONS DE BASE

1.1.1 Source optique

1.1.2 Flux (F)

I.1.3 Angle solide $(\Omega)$

1.1.4 Intensité de source (I)

1.1.5 Luminance de source (L)

1.1.6 Elendue géométrique (G) (pinceau ou faisceau lumineux)

1.1.7 Retour sur la notion de luminance : luminance d'un rayonnement

I.1.8 Systèmes d'unités (SI)

1.2 EXEMPLES DE RELATIONS ENTRE GRANDEURS

1.2.1 Relation entre l'intensité d'une source et son flux

I.2.2 Relation entre la luminance d'une source et son intensité

1.2.3 Relation entre lintensité d'une source et Péclairement du récepteur : loi de Bouguer

1.2.4 Relation entre la luminance d'un rayonnement incident et l'éclairement du récepteur

1.2.5 Cas particulier : les sources à luminance Lniforme

1.2.6 Diffuseurs orthotropes (ou lambertiens)

1.3 RELATIONS PHOTOMÉTRIQUES DANS LES SYSTEMMES OPTIQUES

1.3.1 Transfert de l'étendue géométrique et de la luminance à la réfraction

1.3.2 Rappels d'optique instrumentale

1.3.3 Photométrie des collecteurs de flux

I.3.4 Photométrie des capteurs d'images

1.3.5 Cas des systèmes à champ large

1.4 GRANDEURS SPECTRALES

1.4.1 Position du probleme

1.4.2 Spectre d'un rayonnement

1.4.3 Caractérisation spectrale des surfaces et milieux de propagation

1.4.4 Transmission spectrale d'un milleu de propagation 


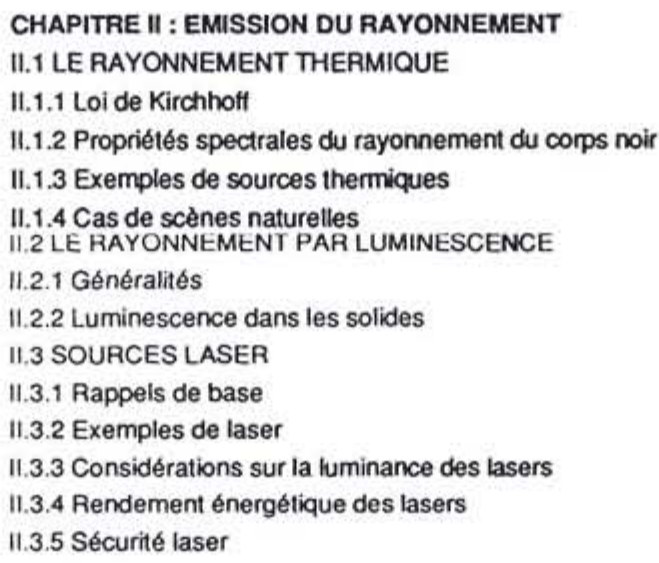




\section{INTRODUCTION}

\section{Evolution de I'optique instrumentale}

L'optique instrumentale s'ouvre actuellement à un nombre de plus en plus important d'applications dans lesquelles l'oeil de l'observateur humain n'est plus directement associé à l'appareil et la conception des systèmes optiques actuels (ou optroniques) fait intervenir, en plus des considérations essentielles en optique et mécanique, des parts grandissantes d'électronique, d'informatique, et de visualisation. Le schéma de la Figure 0-1, représentatif de l'optique instrumentale dans le spatial ou le militaire, permet d'identifier certains des composants de base entrant dans la constitution de nombreux systèmes optiques:

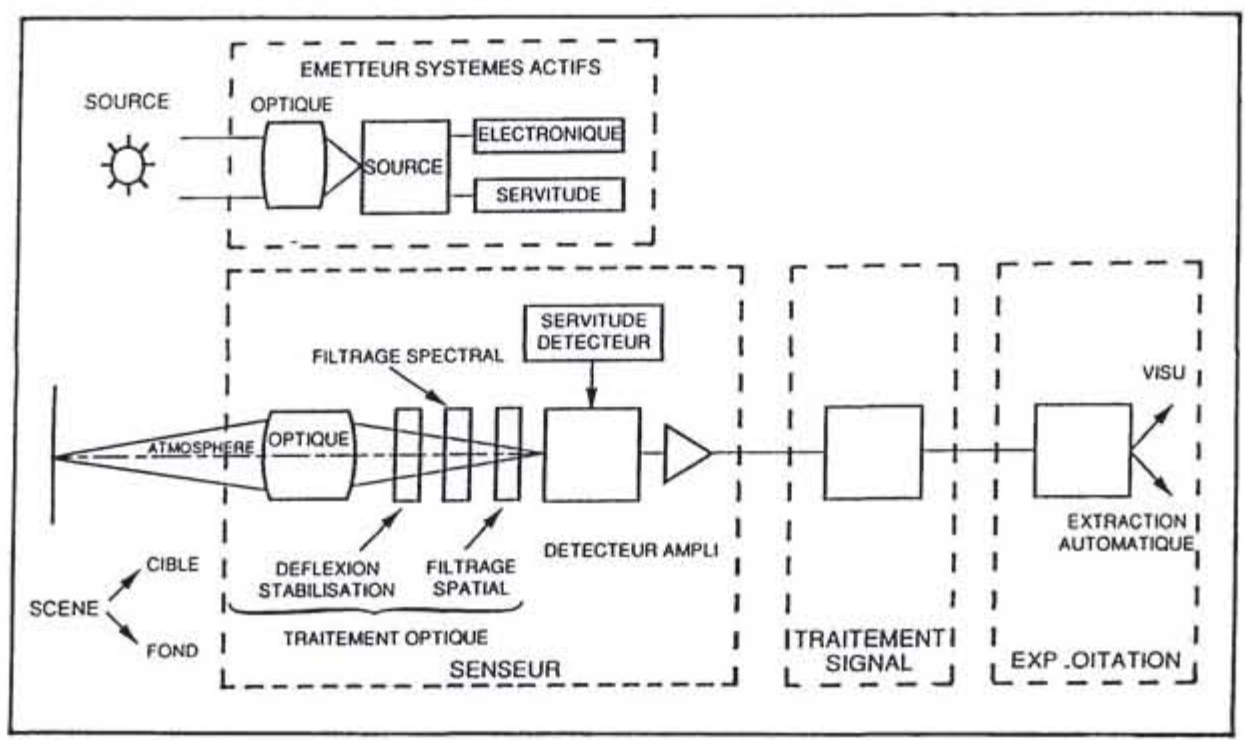

Fig 0-1 : Schéma de principe d'une chaîne optique

De façon générale, on peut dire que la conception de tout système optique s'articule autour de quatre fonctions : l'émission du signal lumineux, sa transmission, sa réception et son mode d'exploitation, avec le souci d'optimiser l'ensemble de la chaîne. Suivant la mission imposée au système sous forme d'un ensemble de spécifications, on s'attachera à définir, entre autres choses, les propriétés spatiales, spectrales et temporelles de la source, les caractéristiques des milieux et surfaces rencontrés par la lumière et leurs effets sur le signal lumineux à détecter, de même que les paramètres de l'optique (champ, ouverture, qualité, ...) en relation avec le détecteur associé. Suivant le mode d'exploitation utilisé, on peut distinguer 3 classes de systèmes optiques:

- Les systèmes visuels, au travers desquels un observateur humain regarde la scène (ex : jumelles, périscopes, ...)

- Les systèmes automatiques, traitant l'information par eux-mêmus, sans intervention humaine (ex : systèmes d'observation de la terre, instrumentation, télécommunications)

- Les systèmes dits "à visualisation" (ex : imagerie télévision de jour, de ntit, imagerie infrarouge).

Le bloc diagramme ci-dessous montre les éléments essentiels de ces différents types de systèmes (Figure 0-2) 


\begin{tabular}{|c|c|c|c|}
\hline Emission & \multicolumn{3}{|c|}{ SOURCE DE LUMIERE } \\
\hline Transmission & \multicolumn{2}{|c|}{ MILIEUX DE PROPAGATION } & \\
\hline Réception & OPTIQUE & & DETECTEUR \\
\hline \multirow[t]{3}{*}{ Exploitation } & & \multicolumn{2}{|c|}{ TRAITEMENT } \\
\hline & & & VISUALSATION \\
\hline & OBSERVATEUR & DECISION & OBSERVATEUR \\
\hline SYSTEMES & VISUELS & AUTOMATIQUES & A VISUALISATION \\
\hline
\end{tabular}

Flg 0-2: Blocs diagrammes de systemes optlques

Rôle de la photométrle en optlque Instrumentale :

La photométrie, ou radiométrie optique, est la discipline concernant la caractérisation théorique et expérimentale des rayonnements dits optiques, c'est-à-dire des radiations électromagnétiques dont les longueurs d'onde sont comprises entre quelques centièmes et quelques centaines de micromètres. Elle a en particulier pour objet de traiter des grandeurs qui définissent ces rayonnements et des lois qui les régissent depuis leur émission jusqu'à leur détection en passant par leur propagation, ainsi que des moyens de mesure correspondants.

Le respect des lois élémentaires de la photométrie constitue, avec celui des lois de l'optique géométrique, l'une des conditions "sine qua non" du bon fonctionnement d'un système optique. La méconnaissance de ces lois de la part du concepteur ou une mise en oeuvre erronée, peuvent entraîner des conséquences néfastes sur les performances du système dans la mesure où ce demier ne recueillera pas ou n'exploitera pas le signal lumineux de façon optimale.

Ainsi, en plus de son rôle en métrologie, la photométrie joue un rôle très important dans la qualité et la compétitivité d'un instrument d'optique. En effet, elle intervient à 2 niveaux clé de la réalisation de tels produits :

- D'une part, elle intervient dès la conception du système, où elle sert de base à l'établissement du budget énergétique des diverses solutions envisagées : son rôle est primordial dans la modélisation de ces solutions, le choix de l'architecture adaptée, la définition des paramètres de base. A ce stade de conception, elle permet d'évaluer théoriquement certaines des performances du système et de définir au plus tốt les procédures et moyens d'essai le concernant.

- D'autre part, la photométrie intervient de façon aussi utile dans les phases de réalisation et d'évaluation expérimentale ("recette") du système grâce à son aspect métrologique (contrôle des composants, tests d'ensemble, vérification du "bilan de liaison".

Ce texte présente les notions de photométrie et de détection optique les plus souvent mises en ceuvre dans les systèmes optiques, que ce soit sous forme de produits industriels ou de montages de laboratoires. Le chapitre I traite des notions de base de la photométrie (grandeurs et relations fondamentales), les chapitres II et III ont pour objets respectifs, les sources et les détecteurs de lumière, et le chapitre IV l'exploitation du signal ("la détection optique"). 


\section{CHAPITRE I}

\section{NOTIONS DE PHOTOMETRIE}

A ses débuts, la photométrie traitait principalement des grandeurs lumineuses perceptibles à l'oeil humain, le capteur optique le plus utilisé. Avec l'usage grandissant de sources et de détecteurs optiques fonctionnant dans un domaine spectral beaucoup plus large que le visible, la photométrie s'est étendue à l'étude des grandeurs énergétiques des ondes électromagnétiques correspondantes $(0,01 \mu \mathrm{m}<\lambda<1$ $\mathrm{mm}$ ). Actuellement, photométrie et radiométrie optique sont des termes synonymes.

Ce chapitre présente les principes de base de la photométrie dite géométrique, c'est-à-dire reposant sur la propagation rectiligne de la lumière (milieux supposés homogènes) et sur les lois de l'optique géométrique. Les résultats ne prennent en compte la diffraction que dans les cas où elle est déterminante dans la performance photométrique d'un système (par exemple dans limage d'un point source). L'influence des intertérences (éclairage cohérent) n'est pas abordée, car elle nécessite dans chaque cas une connaissance plus approfondie du champ électrique (en amplitude et phase) que celle donnée par l'optique géométrique.

La dernière partie de ce chapitre complète ces notions de photométrie géométrique par celle de composition spectrale du rayonnement.

\section{I.1. DEFINITIONS DE BASE}

\section{I.1.1. SOURCE OPTIQUE}

Une source est un générateur de lumière (rayonnement optique) à partir d'autres formes d'énergie, telles que l'énergie électrique (lampes à filament de tungstène, divers types de lasers, diodes, ...), l'énergie électronique (tubes à rayons cath Jdiques, lampes à luminescence, ...), l'énergie thermique (rayonnement en fonction de la température, soleil, ....) ou l'énergie optique (scènes naturelles vues par réflexion ou diffusion de l'éclairement ambian.,...).

On distingue traditionnellement les sources "primaires", qui sont à l'origine de leur rayonnement (par exemple : le soleil) des sources "secondaires", qui n'émettent un rayonnement que dans la mesure où elles sont éclairées par d'autres sources qui les entourent. Les propriétés en émission des sources "primaires" leur sont propres, celles des sources "secondaires" dependent de leur environnement. Par exemple, un paysage observé dans le visible est une source lumineuse dont les caractéristiques sont fonction des conditions météorologiques (ensoleillement, couverture nuageuse, etc.) et de l'heure d'observation (jour/nuit).

Cette distinction simpliste est artificielle car la plupart des sources (en particulier les sources naturelles) émettent ces 2 types de rayonnements, la prépondérance de l'un vis à vis de l'autre étant essentiellement fonction du domaine spectral du système optique utilisé.

Parmi les caractéristiques d'une source les plus utiles dans la conception d'un système optique, on peut citer :

- La géométrie du rayonnement, qui couvre, d̛une part, la géométrie de la source elle-même (on distinguera une source quasi ponctuelle, linéique, surfacique ou volumique) et d'autre part, la directivité du rayonnement (on distinguera les sources émettant dans toutes les directions, dans un demi- $€$ space, ou très directives telles que les lasers). 
- La composition spectrale du rayonnement ou répartition de l'énergie émise en fonction de la longueur d'onde : on parlera de "spectre continu" (couvrant complètement un certain domaine de longueurs d'ondes), quasi monochromatique (centré sur une longueur d'onde), spectre de raies,...

- Le comportement dans le temps : on distinguera les sources dites continues, modulées dans le temps, impulsionnelles (caractérisées par la cadence, la durée et la forme des impulsions).

- Enfin, si la source est artificielle, son rendement énergétique, sa consommation électrique, sa masse, son volume, ...

\subsubsection{FLUX (F)}

Tout rayonnement optique transporte avec lui de l'énergie : pour s'en convaincre, il suffit de rester trop longtemps au soleil, sur la plage. Cette énergie est transportée à une vitesse qui est celle de la lumière dans le milieu de propagation et son débit, par unité de temps, est appelé "Flux énergétique $F_{e}$ " du rayonnement à l'instant considéré. On parle ainsi du flux émis par une source, transmis par un composant optique, réfléchi par une surface, incident sur un détecteur, etc... Ce flux énergétique s'exprime en watts. Pour relier le flux énergétique d'un rayonnement aux caractéristiques physiques de ce cernier, on rappelle que, d'après la mécanique ondulatoire, la lumière est un phénomène à la fois ondulatoire et corpusculaire. Suivant la théorie ondulatoire de la lumière, tout rayonnement peut être considéré comme la superposition d'ondes planes quasi monochromatiques appelées "modes", dont la répartition en fréquences de vibration $\checkmark$ et en directions de propagation dépend de la complexité du rayonnement. Chacun de ces modes est caractérisé par les vecteurs champ électrique $\vec{E}(r, t)$ et champ magnétique $\vec{H}(r, t)$, tels que :

où :

$$
\begin{aligned}
& \vec{E}(\vec{r}, t)=\vec{E}_{0} e^{j(\vec{R} \vec{r}-\omega)} \\
& \vec{H}(\vec{r}, t)=\vec{H}_{0} e^{j(\vec{r} \vec{r}-\omega)}
\end{aligned}
$$

- $\omega$ est la pulsation du mode $(\omega=2 \pi \nu)$

- $k$, le vecteur d'onde désignant la direction de propagation du mode $(k=n \omega / c)$

Si n est l'indice de réfraction du milieu et $c$ la vitesse de propagation de la lumière dans le vide (c $\sim 3.10^{8} \mathrm{~m} / \mathrm{s}$ ), l'énergie lumineuse se propage dans le milieu à la vitesse :

$$
v=c / n
$$

La théorie électromagnétique de la lumière montre que le flux énergétique d'un mode caractérisé par les vecteurs $E$ et $H$ est égal au flux d'un vecteur, caractéristique de cette onde et dénommé vecteur de Poynting S, défini par:

$$
\vec{S}=\vec{E} \wedge \vec{H}
$$

Ce vecteur oscillant à une fréquence double de celle du champ électrique, sa valeur instantanée n'est, en général, pas accessible à la mesure et on montre que le flux énergétique d'un rayonnement est proportionnel à la valeur moyenne de son vecteur de Poynting, égale à :

$$
\left\langle\left|S^{2}\right|\right\rangle=1 / 2 \sqrt{\frac{u}{\varepsilon}}\left|E_{0}\right|^{2}=\left|E_{0}\right|^{2} / 2 Z
$$

où, $\varepsilon, \mu$ et $Z$ sont respectivement la permitivité, la perméabilité magnétique et l'impédance du milieu de propagation (Rappel : l'impédance du vide $Z_{0}$ est égale à $377 \Omega$ ).

D'autre part. d'après la mécanique quantique, la lumière se compose de particules ou photons, dont l'énergie individuelle est proportionnelle à la fréquence $v$ de son onde associée (Théorie Ondulatoire) suivant la relation de Planck:

$$
\mathrm{u}=\mathrm{hv} \quad \text { (h: : Constante de Planck }=6.6210^{-34} \mathrm{~J}-\mathrm{s} \text { ) }
$$


Ainsi, le flux énergétique d'un rayonnement quasi monochromatique correspond à un débit de photons par unité de temps, appelé "flux photonique" Fp et exprimé en nombre de photons par seconde, tels que :

$$
F_{P}=F_{\theta} / u
$$

Enfin, la photométrie a été conduite à la définition d'un troisième type de flux, à cause de l'importance et de la spécificité de l'oeil humain en tant que capteur optique : ce flux Fv, appelé "flux lumineux" (ou "flux visuel") est destiné à quantifier la stimulation visuelle créée par un rayonnement sur un observateur humain dit "standard" et son unité est le lumen (défini au paragraphe III.1).

Le tableau ci-dessous résume les caractéristiques de ces 3 flux qui constituent les bases des 3 systèmes d'unités photométriques (unités énergétiques, photoniques et visuelles).

Tableau 1-1: Flux énergétique, photonique, visuel :

\begin{tabular}{|c|c|c|c|}
\hline Flux & Notation & Définition & Unité \\
\hline ENERGÉTIQUE & $\mathrm{Fe}$ & $\begin{array}{c}\text { Débit } \\
\text { énergétique par } \\
\text { unité de temps }\end{array}$ & WATT (W) \\
\hline PHOTONIQUE & $\mathrm{Fp}$ & $\begin{array}{c}\text { Débit de } \\
\text { photons par } \\
\text { unité de temps }\end{array}$ & $\begin{array}{c}\mathrm{SECONDE}^{-1} \\
\left(\mathrm{~s}^{-1}\right)\end{array}$ \\
\hline VISUEL & $\mathrm{Fv}$ & $\begin{array}{c}\text { "Stimulation } \\
\text { visuelle" }\end{array}$ & LUMEN (Im) \\
\hline
\end{tabular}

Exemples de flux:

- Une lampe à filament de tungstène de $100 \mathrm{~W}$ (consommation électrique moyenne) émet un flux énergétique $\left(F_{e}\right)$ de $90 \mathrm{~W}$, un flux photonique $(F P)$ de $10^{21} \mathrm{~s}^{-1}$ et un flux visuel (Fv) de $1500 \mathrm{~lm}$.

- Un laser NaYag de $500 \mathrm{~W}$ de consommation électrique émet $(a \lambda=1,06 \mu \mathrm{m})$ un flux énergétique de $2 \mathrm{~W}$, un flux photonique de $10^{19} \mathrm{~s}^{-1}$ et un flux visuel nul.

Comme il sera montré plus loin (voir paragraphes 1-4 et III-1), les relations entre les 3 types de flux (énergétique, photonique et visuel) d'un même rayonnement dépendent de la composition spectrale de ce rayonnement.

\section{I.1.3. ANGLE SOLIDE $(\Omega)$}

Définition : l'angle solide sous lequel est vu un objet depuis un point d'observation donné (par exemple, une source depuis un système optique ou vice versa) est le rapport entre l'aire de la calotte sphérique définie par le cône qui s'appuie sur le contour apparent ou silhouette de l'objet (et dont le centre est le point d'observation) et le carré du rayon de cette calotte. L'unité est le stéradian (sr).

\section{Expression mathématique :}

Si les dimensions transversales (c'est-à-dire perpendiculaires à la ligne de visée) de l'objet sont petites vis à vis de sa distance d au point d'observation, l'angle solide correspondant ("angle solide élémentaire") a pour expression :

$$
d \Omega=\frac{d S^{\prime} \cos \theta}{d^{2}}
$$

où dS' : Surface réelle de l'objet et $\theta^{\prime}$ : Angle entre la normale à l'objet et la direction d'observation 


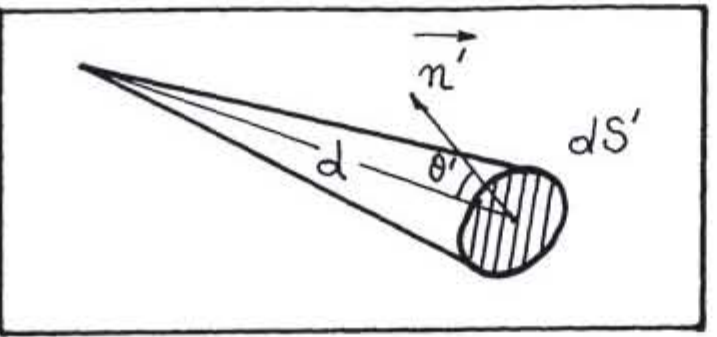

Fig 1 1 : Angle solide
Si l'objet est vu sous l'aspect d'un cercle de rayon apparent $\alpha$ petit (demi-angle au sommet du cône), l'angle solide est alors :

$$
d \Omega=\pi \alpha^{2}
$$

L'angle solide défini par un cône de révolution de demi-angle au sommet fini đaM a pour valeur :

$$
\Omega(\alpha M)=2 \pi(1-\cos \alpha M)
$$

Ainsi, l'angle solide définissant une observation sur un demi-espace est égal à :

$$
\Omega \text { (demi espace) }=2 \pi \mathrm{sr}
$$

et sur l'espace entier :

$$
\Omega \text { (espace entier) }=4 \pi s r
$$

\section{l.1.4. INTENSITE DE SOURCE (I)}

Défınition : L'intensité I d'une source dans une direction donnée est le flux émis par l'ensemble de la source par unité d'angle solide dans la direction considérée. Dans le système énergétique, lintensité s'exprime en W/sr.

Si $d F_{s}\left(u, v, d \Omega_{s}\right)$ est le flux émis par la source $d$ ans l'angle solide élémentaire $d \Omega_{s}$ centré sur la direction de coordonnées angulaires $u$ et $v$, l'intensité de la source dans cette direction est donc :

$$
I(u, v)=d F_{;}(u, v, d \Omega s) / d \Omega s
$$

Indlcatrice d'intenslté . L'intensité d'une source est en général fonction de la direction d'émission : le comportement angulaire d'une source est défini par son "indicatrice en intensité", surface entourant la source et délimitée par le lieu de l'extrémité du vecteur $\vec{T}(u, v)$, son origine étant maintenue fixe, par exemple au centre de la source.

Les sources d'intensité constante quelle que soit la direction d'émission (cas des étoiles) sont dites "isotropes" et leur indicatrice d'intensité est une sphère. Les sources très directives (ex : laser Hélium Néon) ont une indicatrice d'intensité très allongée le long de leur axe d’émission.

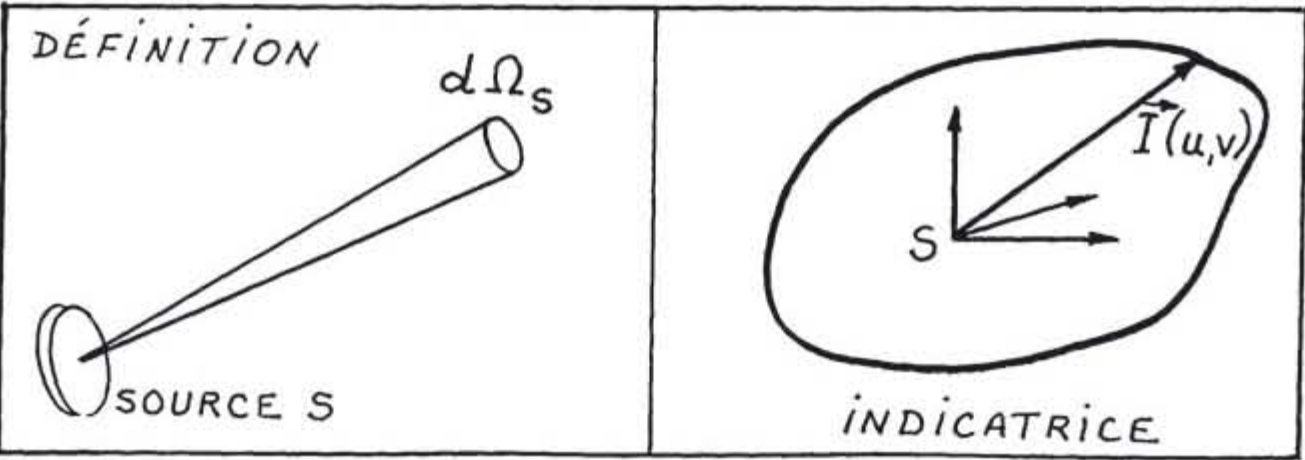

Fig 1-2: Intensité de la source 
Remarques sur la notion d'intensité :

- La notion d'intensité de source caractérise le rayonnement de l'ensemble de la source dans une direction, mais ne donne pas de renseignements sur la source elle-même (forme, dimensions, présence éventuelle de zones privilégiées en émission ...)

- Pour mesurer expérimentalement l'intensité d'une source, on veillera à ce que la résolution angulaire du montage soit compatible des fluctuations angulaires de l'indicatrice d'intensite.

\section{I.1.5. LUMINANCE DE SOURCE (L)}

Si l'on désire caractériser le rayonnement d'une source de façon plus approfondie que par la simple connaissance de son intensité, on décompose cette source en un ensemble de petits éléments indépendants les uns des autres (on ne considère ici que le cas de sources incohérentes). Par exemple, isolons un petit élément de la source autour d'un point $A(x, y, z)$ au moyen d'un diaphragme d'aire dAs qui sera soit plaqué contre la source si cette demière a une géométrie bien définie (par exemple, source surfacique), soit interposé entre le point d'observation et la source elle-même si la géométrie de cette dernière est mal connue (ce qui est la cas du ciel, par exemple).

II est alors possible de définir, d'après les paragraphes précédents, l'intensité de ce petit élément de source d'aire dAs dans la direction $(u, v)$ par l'expression suivante :

$$
d K x, y, z, u, v)=d^{2} F S(x, y, z, u, v, d \Omega S) / d \Omega S
$$

si $d^{2} F s$ représente le flux rayonné par le petit élément d'aire $d A s(x, y, z)$ dans l'angle solide d $\Omega$ s centré sur la direction $(u, v)$.

Par définition, la luminance de zone de source localisée autour du point de coordonnées $(x, y, z)$ et dans la direction $(u, v)$ est lintensité de la source par unité d'aire apparente dans cette direction, soit mathématiquement:

$$
L(x, y, z, u, y)=d k x, y, z, u, y) / d A s \cos \theta s
$$

où $\theta$ s est l'angle entre la normale locale à la source (ou au diaphragme plan utilisé pour délimiter la source) et la direction d'émission, d'où :

$$
L(x, y, z, u, y)=d^{2} F S(x, y, z, u, v, d \Omega S) / d A s \cos \theta s d \Omega S
$$

L'unité énergétique de la luminance est le $\mathrm{Wm}^{-2} \mathrm{sr}^{-1}$

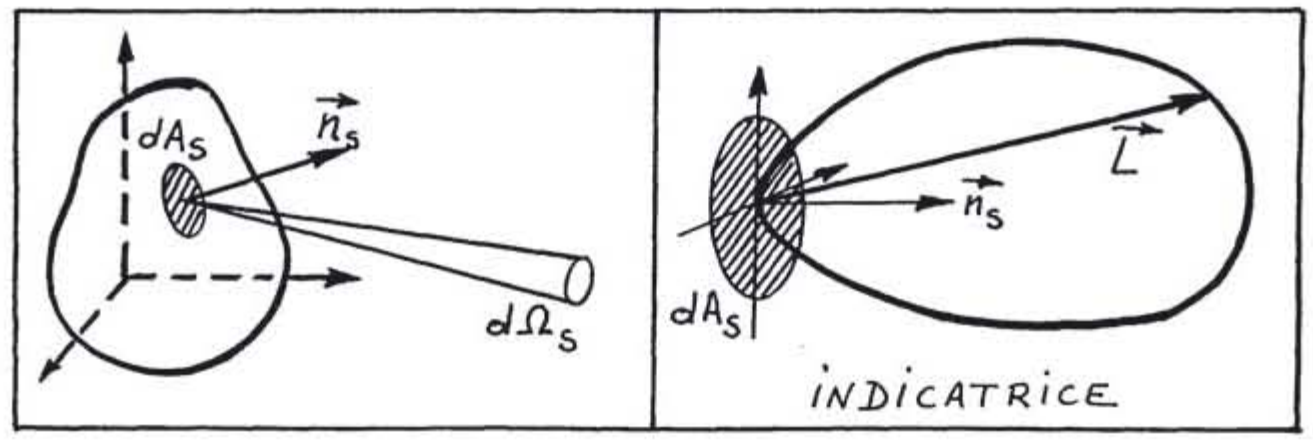

Flg 13 : Luminance de la source 


\section{l.1.6. ETENDUE GEOMETRIQUE (G)}

Cas d'un pinceau lumineux :

En simplifiant les notations, il ressort du paragraphe précédent que le flux élémentaire $d^{2} F s$ érnis par le petit élément de source d'aire $d A s$ dans l'angle solide $d \Omega$ s est égal à :

$$
d^{2} F s=L S d A s \cos \theta s d \Omega S
$$

La quantité $d^{2} G=d A S \cos \theta S d \Omega$ s caractérisée par la surface émissive de la source et l'angle solide d'émission est appelée "étendue géométrique" du pinceau de rayons à l'émission.

Si l'angle solide d'émission de ce pinceau est défini par la silhouette d'un récepteur d'aire dAR situé à une distance $d$ de la source, on constate que l'étendue géométrique d'un pinceau défini par 2 diaphragmes d'aires dAs et dAR situés à la distance mutuelle d peut s'exprimer de 2 autres façons :

$$
d^{2} G=\frac{d A_{S} \cos \theta_{S} d A_{R} \cos \theta_{R}}{d^{2}}=d A_{R} \cos \theta_{R} d \Omega_{A}
$$

où $d_{\Omega R}$ est l'angle solide sous lequel est vu l'élément de source dAs depuis le second diaphragme. En pratique, l'utilisation préférentielle de l'une ou l'autre de ces 3 formules sera dictée par la facilité de son expression mathématique en fonction des paramètres géométriques définissant les 2 diaphragmations.

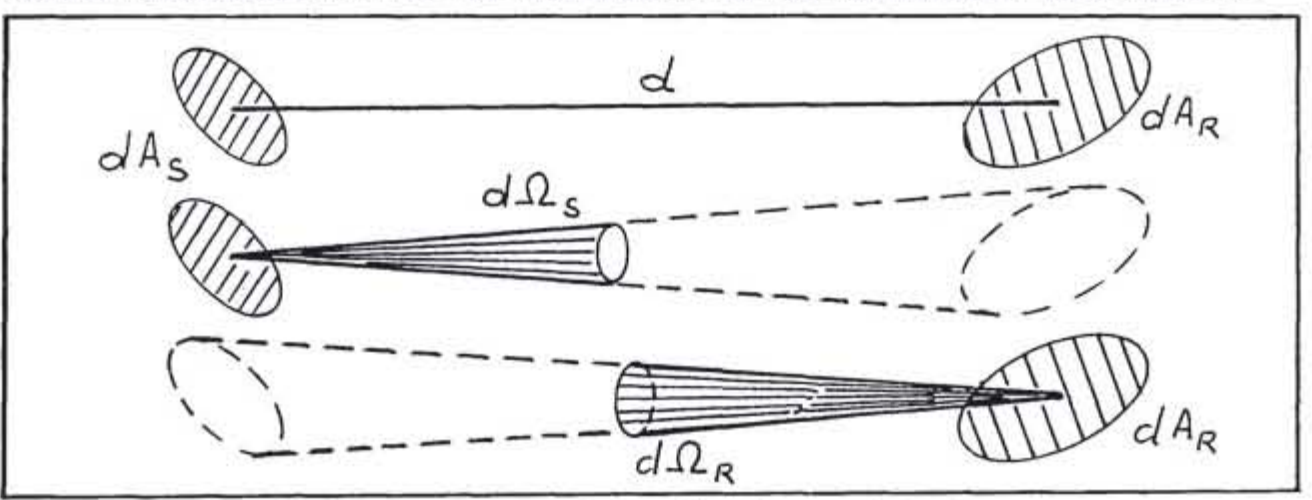

Flg 1 -4 : Etendue géométrlque d'un plnceau de lumlère

Cas d'un faisceau lumineux :

Les diaphragmes définissant l'émetteur et le récepteur de flux se voient mutuellement sous des angles solides finis, 'Tétendue géométrique G correspondant à ce faisceau se calcule en sommant les contributions élémentaires en étendue géométrique des pinceaux qui le constituent, d'où

$$
G=\int_{\text {surfacedurécopteur }} \int_{\text {surfacedelasource }} d^{2} G
$$

Le calcul peut être plus ou moins ardu suivant la configuration géométrique des 2 diaphragmes. Dans le cas, fréquent en optique, oủ le système possède une symétrie de révolution autour de son axe optique, l'expression mathématique de l'étendue géométrique $\mathrm{G}$ se calcule assez simplement en décomposant l'angle solide sous lequel est vu l'un des diaphragmes depuis l'autre en une succession d'angles solides élémentaires annulaires, compris entre les angles $\theta$ et $\theta+d \theta$ et dont la valeur est :

$$
d \Omega=2 \pi \sin \theta \alpha \theta
$$


L'étendue géométrique s'écrit dans ce cas :

$$
\begin{aligned}
G & =\int_{\text {diaphragme } 1} \int_{\text {diaphragme } 2} d A \cos \theta \alpha \Omega=\int_{\text {Aire } A_{R}} d A_{R} \int_{0}^{\theta \mu} 2 \pi \sin \theta \cos \theta d \Omega \\
& =\pi A_{R} \sin ^{2} \theta_{M}
\end{aligned}
$$

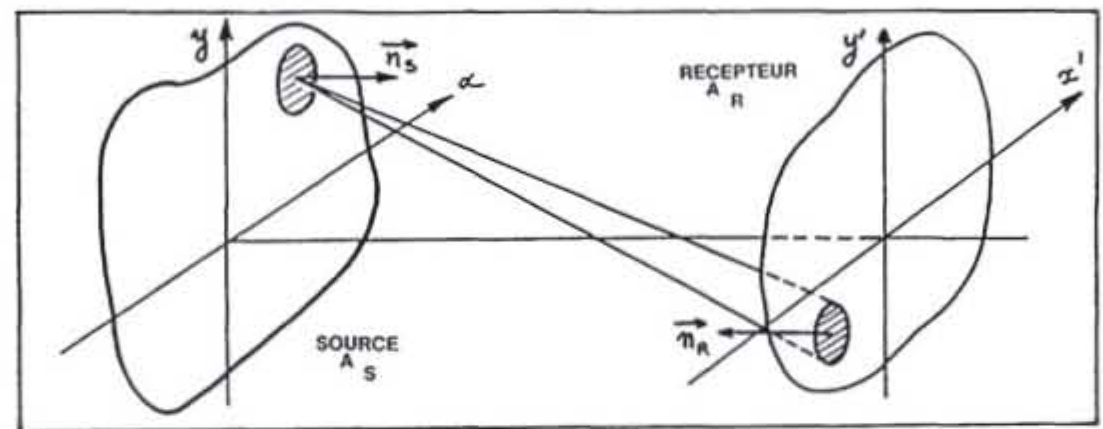

Flg I-5 : Etendue géométrique d'un falsceau

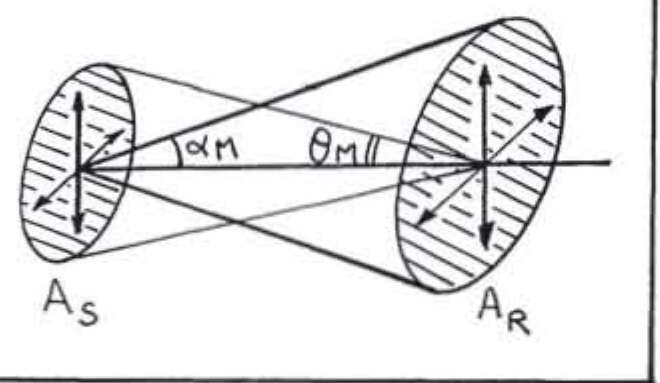

Flg 1-6 : Falsceau à symétrie cylindrique

De même, si le récepteur est vu depuis la source sous un cône de demi angle au sommet aM. l'étendue géométrique s'écrit alors :

$$
G=\pi \text { As } \sin ^{2} \alpha_{M}
$$

D'où les 2 expressions d'un faisceau à symétrie cylindrique :

$$
G=\pi A_{A} \sin ^{2} \theta_{M}=\pi A_{S} \sin ^{2} \alpha_{M}
$$

\subsubsection{RETOUR SIIR LA NOTION DE LUMINANCE : LUMINANCE D'UN RAYONNEMENT}

Considérons à nouveau le pinceau lumineux défini par un élément de source dAs et un élément de récepteur $\mathrm{dAR}_{\mathrm{R}}$. Si ce pinceau ne subit aucune atténuation le long de son trajet, le flux reçu par le récepteur et provenant de la source dAs est égal au flux $\mathrm{d}^{2} \mathrm{FS}$ émis par cet élément de source dans l'angle solide définissant le récepteur, soit $\mathrm{dFR}=\mathrm{dFS}$

d’où

$$
L_{R} d A_{R} \cos \theta_{R} d \Omega_{R}=L S d A_{S} \cos \theta_{S} d \Omega_{S}
$$

Le terme Ls défini au paragraphe L.5.1 caractérisait la luminance du rayonnement de la source au niveau de l'émission. Le terme LR est appelé luminance du rayonnement dũ à la source au niveau du récepteur. II est conservatif, dans un milieu homogène sans perte, tout au long de chaque pinceau, depuis la source jusqu'au récepteur. 
Si le milieu de propagation introduit des pertes le long du trajet, ces demières se traduisent par le facteur de transmission (géométrique) du milieu sur le trajet considéré, rapport entre les luminances du rayonnnement entre les 2 points extrêmes de ce parcours :

$$
\tau\left(X_{1}, X_{2}\right)=L\left(X_{2}\right) / L\left(X_{1}\right)
$$

La luminance du rayonnement dô à une source en un point quelconque d'un pinceau lumineux est aussi appelée luminance de la source en ce point.

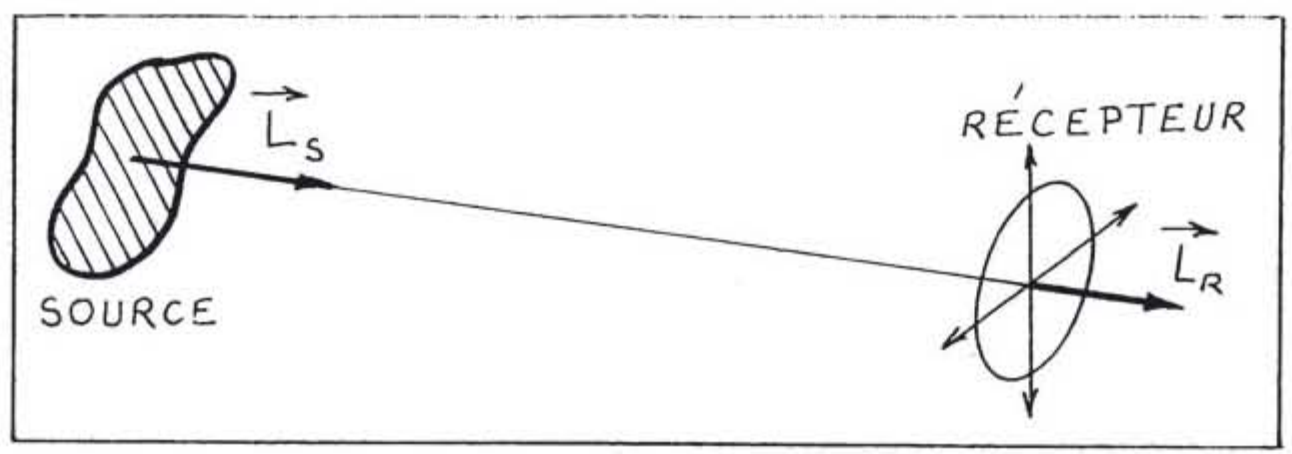

Flg 1-7: Luminance de rayonnement

1.1.8. AUTRES GRANDEURS

Exitance de source $\left(M\right.$ en $\left.W / \mathrm{m}^{2}\right)$

L'exitance $M$ d'une surface en un point est la densité de flux emis dans un demi-espace par unité de surface émissive :

$$
M(x, y)=d F(x, y) / d A_{S}
$$

L'exitance est aussi appelée Emittance (ou Radiance)

Eclairement de surface réceptrice $\left(E\right.$, en $\left.W / \mathrm{m}^{2}\right)$

L'éclairement $E$ d'une surface en un point est la densité de flux incident sur cette surface par unité d'aire, au point considéré.

Quantlté de lumlère ( $\mathcal{F}$ en Joules)

La quantité de lumière $\mathcal{F}$ définie par un flux $F$ et une durée t est lintégrale de ce flux sur la durée $t$.

Exposition ( $\mathcal{E}$ en $\mathrm{J} / \mathrm{m}^{2}$ )

L'exposition $\mathcal{E}$ d'une surface pendant une durée t est l'intégrale de son éclairement pendant cettz durée. 


\section{I.1.9. SYSTEMES D'UNITES (SI)}

Le tableau suivant résume les unités à employer dans les 3 systèmes photométriques (énergétique, photonique et visuel) :

\begin{tabular}{|c|c|c|c|}
\hline \multirow[t]{2}{*}{ Grandeur } & \multicolumn{3}{|c|}{ Unités } \\
\hline & Energátiques & Photoniques & Visuelles \\
\hline FLUX & W & $\mathrm{s}^{-1}$ & lumen (Im) \\
\hline INTENSITÉ & $\mathrm{W} \mathrm{sr}{ }^{-1}$ & $s^{-1} s r^{-1}$ & Candela (cd) \\
\hline LUMINANCE & $W \mathrm{~m}^{-2} \mathrm{sr}^{-1}$ & $\mathrm{~s}^{-1} \mathrm{~m}^{-2} s r^{-1}$ & $\operatorname{cd} \mathrm{m}-2$ \\
\hline EXITANCE & $W m^{-2}$ & $\mathrm{~s}^{-1} \mathrm{~m}^{-2}$ & $1 \mathrm{~m} \mathrm{~m}^{-2}$ \\
\hline ECLAIREMENT & $W m^{-2}$ & $\mathrm{~s}^{-1} \mathrm{~m}^{-2}$ & lux \\
\hline $\begin{array}{l}\text { QUANTITÉ DE } \\
\text { LUMIĖRE }\end{array}$ & $\mathrm{J}$ & Nombre de photons & Im s \\
\hline
\end{tabular}

\section{I.2. EXEMPLES DE RELATIONS ENTRE GRANDEURS}

II arrive souvent que tel ou tel composant soit spécifié par le fournisseur selon une grandeur photométrique difficilement utilisable par le concepteur de système optique, qui doit être capable de transposer les données dont il dispose, en paramètres utiles à la résolution de son problème. Ce paragraphe présente les relations de passage entre grandeurs photométriques dans un milieu homogène. Le passage au moyen d'un système optique sera traité au paragraphe I.3.

\subsubsection{RELATION ENTRE L'INTENSITE D'UNE SOURCE ET SON FLUX}

D'après la définition de l'intensité I d'une source, le flux $F_{\Omega}$ émis par cette source dans un angle solide $\Omega$ sera :

$$
F \Omega=\int_{\Omega} I(u, v) d \Omega
$$

Le flux total émis par la source dans tout l'espace sera $F_{4 \pi}$. Si la source est isotrope, la mesure de son intensité dans une seule direction suffit pour connaître le flux total émis par cette source, puisque :

$$
F_{4 \pi}=4 \pi l_{0}
$$

\subsubsection{RELATIONS ENTRE LA LUMINANCE D'UNE SOURCE ET SON INTENSITE}

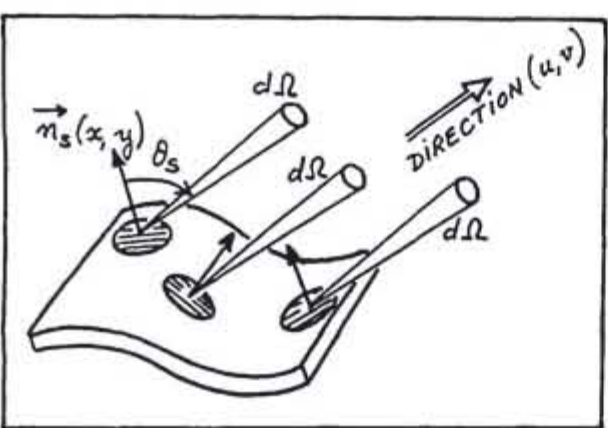

Fig 1-8 : Luminance et Intensité
Considérons une source non plane, de luminance $L(x, y, u, v)$. Son intensité dans la direction $(u, v)$ se calcule en décomposant la surface émettrice en zones élémentaires d'aire dAs.. Le flux $d^{2} \mathrm{FS}$ émis par chacune d'entre elles dans l'angle solide $d \Omega$ centré sur cette direction est égal à :

$$
d^{2} F s=L(x, y, z, u, y) d A s \cos \theta s d \Omega
$$

Le flux émis par l'ensemble de la source dans cet angle solide est alors :

et l'intensité :

$$
d F S=\int_{\text {source }} 2(x, y, z, u, y) d A s \cos \theta s d \Omega
$$

$$
k(u, v)=\frac{d F S}{d \Omega}=\int_{\text {source }} L(x, y, u, y) \cos \theta s d A S
$$




\subsubsection{RELATION ENTRE INTENSITE DE SOURCE ET ECLAIREMENT DE RECEPTEUR : LOI DE BOUGUER}

Si l(u,v) est l'intensité de la source en direction du détecteur, T la transmission du milieu qui les sépare sur une distance d et $\theta$ l'angle d'obliquité du récepteur vis à vis de la direction de visée, l'éclairement du récepteur est (loi dite de Bouguer) :

$$
E_{R}=\tau \mid(u, v) \cos \theta_{R} / d^{2}
$$

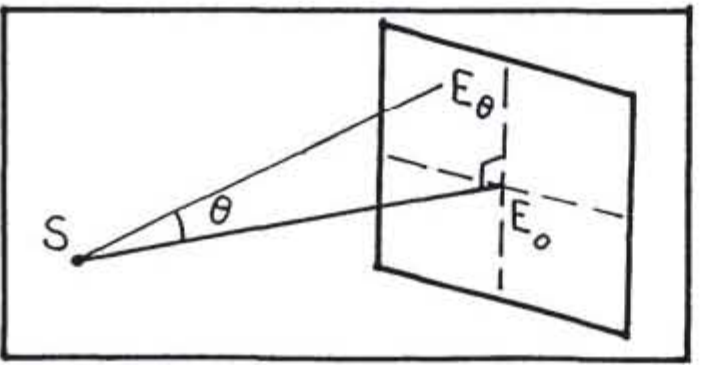

Exercice : Calcul de l'éclairement relatif d'un plan face à une source quasi ponctuelle isotrope.

$$
\begin{aligned}
& F_{-0}=I_{0} / d^{2} \\
& E_{\theta}=\frac{l_{0} \cos \theta}{\left(d_{0} / \cos \theta\right)^{2}}=E_{0} \cos ^{3} \theta
\end{aligned}
$$

Fla 19 : Eclalrement d'un plan (source ponctuelle)

\subsubsection{RELATION ENTRE LA LUMINANCE INCIDENTE ET L'ECLAIREMENT DU RECEPTEUR}

1er cas : Eclairement d'une surface provenant d'un rayonnement à luminance constante LR dans un angle solide faible $(\Delta \Omega R)$

L'éclairement de la surface pour une incidence du rayonnement sous l'angle $\theta_{R}$ est égal à :

$$
\Delta E_{R}=L_{R} \cos \theta_{R} \Delta \Omega_{R}
$$

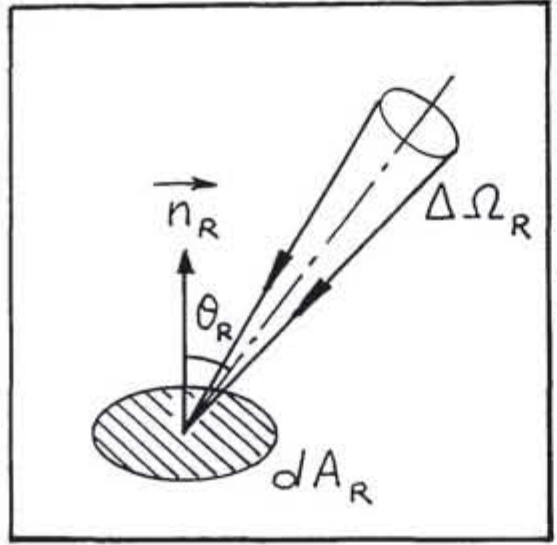

Flg $1-10$ : Eclairage par pinceau uniforme
Exemple:

Calculer l'éclairement d'un plan perpendiculaire aux rayons du soleil. Le soleil est vu depuis la terre sous un diamètre apparent de 30' d'arc et sa luminance apparente (hors atmosphère) est de $L_{R}=2,210^{7} \mathrm{~W} \mathrm{~m}^{-2} \mathrm{si}^{-1}$ (énergétique) et $210^{9} \mathrm{Cd} \mathrm{m}^{-2}$ (visuelle).

L'éclairement exoatmosphérique de la terre dans un plan de front vis à vis des rayons solaires est donc:

$$
\Delta E_{R}=L_{R} \Delta \Omega R
$$

L'angle solide sous lequel on voit le soleil depuis la terre est

$$
\Delta \Omega_{R}=\pi \alpha^{2} \text { avec } \alpha=15^{\prime}=4,510^{-3} r d
$$

d'où l'éclairement dû au soleil :

$E e=1450 \mathrm{~W} / \mathrm{m}^{2}$ (énergétique)

$E v=130000$ lux (visuel) 
2ème cas : Cas général d'un rayonnement hémisphérique de luminance non uniforme.

Le rayonnement qui éclaire la surface réceptrice depuis une demi sphère est à décomposer en pinceaux élémentaires

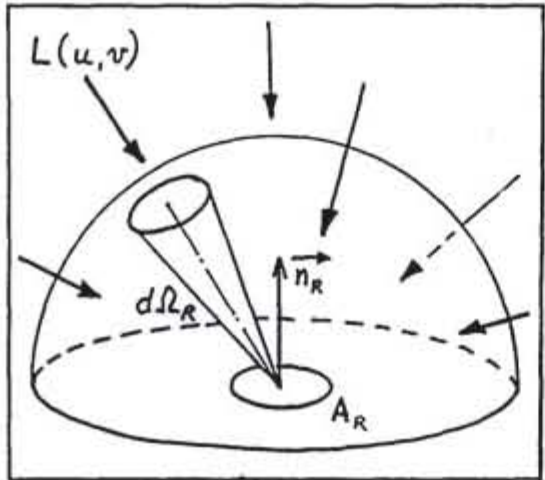
caractérisés par une luminance apparente $L R(u, v)$ et dont chaque contribution en éclairement de la surface est égale à

$$
d E_{R}(u, v)=L A(u, v) \cos \theta_{R} d \Omega A
$$

d'où l'éclairement total de cette surface dô à l'ensemble du rayonnement incident :

$$
E_{R}=\int_{1 / 2 \theta s p a c e} L_{R}(u, v) \cos \theta_{R} \alpha \Omega_{R}
$$

Flg l-11 : Eclalrage hémlsphérique

\subsubsection{CAS PARTICULIER (mais extrềnement général) : LES SOURCES A LUMINANCE UNIFORME}

D'après le paragraphe I.1.5, la luminance du rayonnement émis par une source est un paramètre qui dépend à la fois de la zone de source (de coordonnées $x, y, z$ ) et de la direction d'émission (de coordonnées angulaires u,v). II existe un certain nombre de sources, en particulier, de sources naturelles (telles que le ciel bleu dans des directions loin du soleil, un ciel très couvert, un mur, ..) dont la luminance peut être considérée uniforme :

- elle est dite uniforme angulairement si elle est indépendante de la direction d'émission mais varie d'un point à l'autre de la source :

$$
L(x, y, z, u, v)=L(x, y, z)
$$

- elle est dite uniforme spatialement et angulairement si elle est indépendante de la zone d'émission sur la source et de la direction d'émission :

$$
L(x, y, z, u, v)=L
$$

Les propriétés spécifiques en luminance de telles sources permettent d'établir des relations simples entre grandeurs caractéristiques da leur rayonnement, particulièrement utiles dans la modélisation de systèmes optiques.

Relation entre luminance et exitance :

Dans le cas d'une source uniforme en luminance, la symétrie cylindrique de l'émission permet de décomposer l'angle solide d'émission $(2 \pi s r)$ de chaque zone élémentaire en anneaux (voir le paragraphe I.1.6), d'où :

$$
\begin{aligned}
M(x, y) & =\int_{1 / 2 \theta s p a c \theta} L(x, y, z, u, y) \cos \theta d \Omega \\
& =\int_{0}^{\pi / 2} 2 \pi \sin \theta \cos \theta d \theta=\pi L(x, y)
\end{aligned}
$$


Relation entre luminance et Intensité :

Si la source est uniforme spatialement et angulairement, l'expression générale de l'intensité de la source en fonction de sa luminance (voir paragraphe I.2.2) devient :

$$
\begin{aligned}
I(u, v) & =\int_{\text {surfacodelasource }} L\left(x, y, u, v \cos \theta s d A S=L \int_{\text {source }} d A s \cos \theta s\right. \\
& =\mathrm{L} A_{\text {app }}(u, v)
\end{aligned}
$$

oủ $A_{\text {app }}(u, v)$ est l'aire apparente de la source dans la direction $(u, v)$ consiuérée.

Exemples:

- Calcul de l'intensité d'une source sphérique de rayon r à luminance uniforme ( $L$ )

La surface apparente de la sphère dans chaque direction est celle de son disque ( $\pi / 2)$ d'où :

$$
I_{\text {sphère }=L_{i}=\pi R^{2} L}
$$

- Calcul de l'intensité d'un disque plan d'aire As de luminance uniforme L dans la direction $\Theta$ par rapport à la normale:

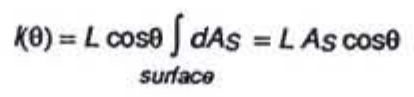

Relation entre luminance de rayonnement et éclairement de surface réceptrice :

Soit une surface plane éclairée par un rayonnement de luminance uniforme à l'intérieur d'un angle solide fini de demi-angle au sommet $\Theta \mathrm{M}$ de révolution autour de la normale à la surface. L'éclairement $E_{R}$ de cette surface est :

$$
\begin{aligned}
& E_{R}=d F_{R} / d A_{R}=\int_{M}^{\theta_{M}} L_{R} \cos \theta 2 \pi \sin \theta d \theta \\
& E_{R}=\pi L_{R} \sin ^{2} \theta_{M}
\end{aligned}
$$

Si la luminance apparente du rayonnement est uniforme sur tout le demi-espace, il vient :

$$
E_{R}=\pi L_{R}
$$

Exemple : Calcul de l'éclairement visuel du sol dû à un ciel bleu supposé uniforme, de luminance

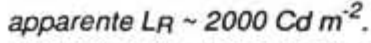

D'après la relation ci-dessus, léclairement au sol est :

$$
\begin{aligned}
E_{\text {sol }} & =\pi L_{\text {ciel }} \quad \text { (où Lciel est la luminance du rayonnement du ciel au niveau du sol) } \\
& \sim 6300 \text { lux }
\end{aligned}
$$

\subsubsection{DIFFUSEURS ORTHOTROPES (OU LAMBERTIENS)}

Un diffuseur parfait (en réflexion ou en transmission) est une surface qui réfléchit ou transmet tout le flux incident avec une luminance qui dépend uniquement de son éclairement, quelles que soient la direction et la directivité du rayonnement incident et la direction d'observation du rayonnement réfléchi ou transmis. Par définition, leur exitance en réflexion ou transmission est égale à leur éclairement et, puisque leur luminance est uniforme, cette dernière est égale à (voir paragraphe 1.2.5) :

$$
\mathrm{L}=\mathrm{M} / \pi=\mathrm{E} / \pi
$$


Contrairement aux surfaces optiques (telles que miroirs, dioptres, etc...) qui restituent un rayonnement dans la direction imposée par les lois de l'optique géométrique (lois de Descartes - Snell), les diffuseurs parfaits sont dénommés "surfaces sans mémoire" : l'observation du rayonnement qu'elles diffusent ne permet pas de connaitre la direction d'incidence du faisceau incident, qu'elles ont "oubliée" dans le processus de diffusion.

En pratique, les diffuseurs réels réfléchissent (ou transmettent) seulement une fraction $R$ (ou $\tau$ ) du rayonnement incident et leur luminance dépend plus ou moins des directions d'illumination et de réemission. Si leur luminance est uniforme, ces surfaces sont dites orthotropes ou lambertiennes. La luminance, constante quelle que soit la direction d'émission, est alors liée à l'éclairement $E$ par le facteur de réfléxion diffuse $\mathcal{R}_{d}$ (ou transmission diffuse $\mathcal{T}_{d}$ ) de la surface par la relation (dite relation de Lambert)

$$
\begin{array}{ll}
L=\tau_{d} E / \pi & \text { (en réflexion) } \\
L=\tau_{d} E / \pi & \text { (en transmission) }
\end{array}
$$

Exemple:

Calcul de la luminance visuelle en réflexion diffuse de la pleine lune (de facteur de réflexion diffuse ou albedo $~ 0,07$ dans le visible) :

$$
\text { Lv(lune })=T_{\text {atm }} R_{d} E / \pi \quad 0,75 \times 0,07 \times 1,310^{5} / \pi-2300 \mathrm{Cd} / \mathrm{m}^{2}
$$

Note:

La qualité lambertienne d'une surface diffusante, par exemple en réfléxion, (bonne uniformité en luminance) dépend essentiellement de sa rugosité (profondeur et largeur moyenne des fluctuations de la surface) par rapport à la longueur d'onde du rayonnement incident. II faut noter que cette rugosité apparente décroit en fonction de l'angle d'incidence du faisceau. Une surface, considérée comme lambertienne sous incidence proche de la normale, perd en général son caractère orthotrope sous incidence de plus en plus rasante (sa rugosité apparente décroît).

\subsection{RELATIONS PHOTOMETRIQUES DANS LES SYSTEMES OPTIQUES}

Le paragraphe précédent traitait des relations photométriques pour un rayonnement dans un espace donné, supposé homogène. Le présent paragraphe traite des relations entre grandeurs photométriques en présence de systèmes optiques.

\subsubsection{TRANSFERT DE L'ETENDUE GEOMETRIQUE ET DE LA LUMINANCE A LA REFRACTION}

On a montré précédemment limportance des notions d'étendue géométrique et de luminance de rayonnement qui se conservent dans un milieu homogène sans perte. Les systèmes optiques comportant en général des composants d'indices différents, il importe de suivre l'évolution de ces 2 paramètres (étendue géométrique et luminance) tout au long du trajet parcouru par un rayonnement, pour évaluer par exemple le flux dans l'espace image du système optique. 
Soit un dioptre séparant 2 milieux d'indices de réfraction $n$ et $n$ ', sur lequel un pinceau lumineux d'étendue géométrique connue $d^{2} G$ subit une réfraction. Pour calculer de façon simple la valeur de l'étendue géométrique de ce pinceau après réfraction dans le second milieu, considérons que l'étendue du pinceau initial est limitée en aval par un petit diaphragme (dit diaphragme d'ouverture) d'aire $d A_{0}$ posé sur le dioptre, et en amont, par un petit diaphragme (dit diaphragme de champ) perpendiculaire au pinceau et vu depuis le précédent sous des angles définis entre les incidences $i$ et $i+$ di et entre les latitudes $\Lambda$ et

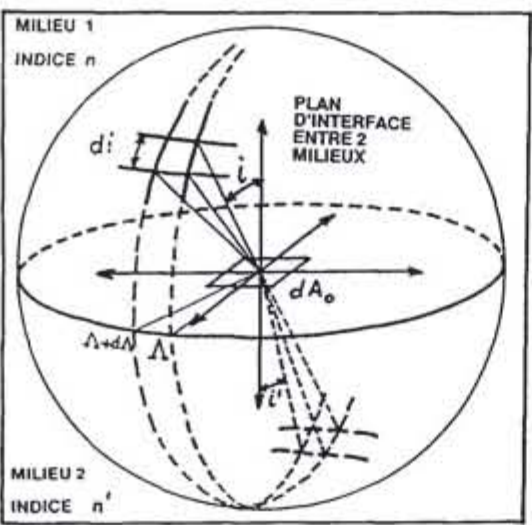

Fig $1-12$ : Etendue et réfraction $\Lambda+d \Lambda$ (voir Figure l-12).

L'angle solide sous lequel apparait le diaphragme de champ depuis le diaphragme d'ouverture est :

$$
d \Omega=d i[\sin i x d \Lambda]
$$

L'étendue géo nétrique du pinceau dans le premier milieu s'écrit alors :

$$
\begin{aligned}
& d^{2} G=d A 0 \cos i d \Omega \\
& =d A 0 \sin i \cos i d i d \Lambda
\end{aligned}
$$

L'étendue géométrique du pinceau dans le second milieu peut s'exprimer, de façon analogue par:

$$
d^{2} G^{\prime}=d A 0 \sin i^{\prime} \cos i^{\prime} d i^{\prime} d \Lambda^{\prime}
$$

La prise en compte des lois de Descartes - Snell à la réfraction:

$$
n^{\prime} \sin i^{\prime}=n \sin i\left(\Rightarrow n^{\prime} \cos i^{\prime} d i^{\prime}=n \cos i \text { di }\right)
$$

montre que: $\quad n^{\prime 2} d^{2} G^{\prime}=n^{2} d^{2} G \Rightarrow n^{\prime 2} G^{\prime}=n^{2} G$

Il en résulte que l'étendue géométrique $\sigma^{2} G$ d'un pinceau (ou faisceau) lumineux ne se conserve pas à la réfraction, mais seulement le produit $n^{2} \mathrm{G}$, appelé "étendue optique".

Si $\tau_{g}$ est la transmission en luminance (ou dans le sens de l'optique géométrique) du dioptre dans la zone de passage du pinceau lumineux, le flux optique transporté par ce finceau dans le second milieu peut s'écrire :

soit :

$$
d^{2} F^{\prime}=\tau_{g} d^{2} F
$$

$$
L^{\prime} d^{2} G^{\prime}=\tau_{g} L d^{2} G
$$

oủ L' est la luminance du rayonnement dans le second milieu. II ressort donc que les luminances dans le premier et le second milieu sont reliées par:

$$
L^{\prime} / \mathrm{n}^{\prime 2}=\tau_{g} L / \mathrm{n}^{2}
$$

Nota : si les milieux extrêmes du système optique sont identiques, la luminance du rayonnement dans l'espace final est :

$$
\mathrm{L}^{\prime}=\tau_{\mathrm{g}} \mathrm{L}
$$

Dans le cas de la réflexion du rayonnement sur un miroir, de facteur de réflexion géométrique $\Re_{g}$, on a :

$$
L^{\prime}=R_{g} L
$$




\subsubsection{RAPPELS D'OPTIQUE INSTRUMENTALE}

\section{Dlaphragmes d'un système optique}

L'étendue géométrique du faisceau lumineux transmissible par un instrument d'optique est, dans les cas les plus simples, limitée par la présence de 2 diaphragmes : le premier, dit "diaphragme d'ouverture", est en général physiquement implanté dans le dispositif optique lui-même et définit l'angle solide du faisceau (ou pinceau) utile émis par un point de la source (par exemple, le point de la source situé sur l'axe du système). Ce diaphragme d'ouverture pourra être l'objectif d'une lunette de visée, le diaphragme à iris d'un appareil photographique, ....).

Le second diaphragme dit "de champ" est déterminé suivant les cas par le bord de la source (dans respace objet du système) ou par les dimensions physiques du détecteur (dans l'espace image). C'est lui qui impose une limite aux rayons provenant hors de l'axe du système (ou bord du champ).

Les images respectives du diaphragme d'ouverture dans les espaces amont (objet) et aval (image) du système sont appelées pupilles d'entrée et de sortie du système. Celles du diaphragme de champ dans les mêmes espaces sont dénommées lucarnes d'entrée et de sortie

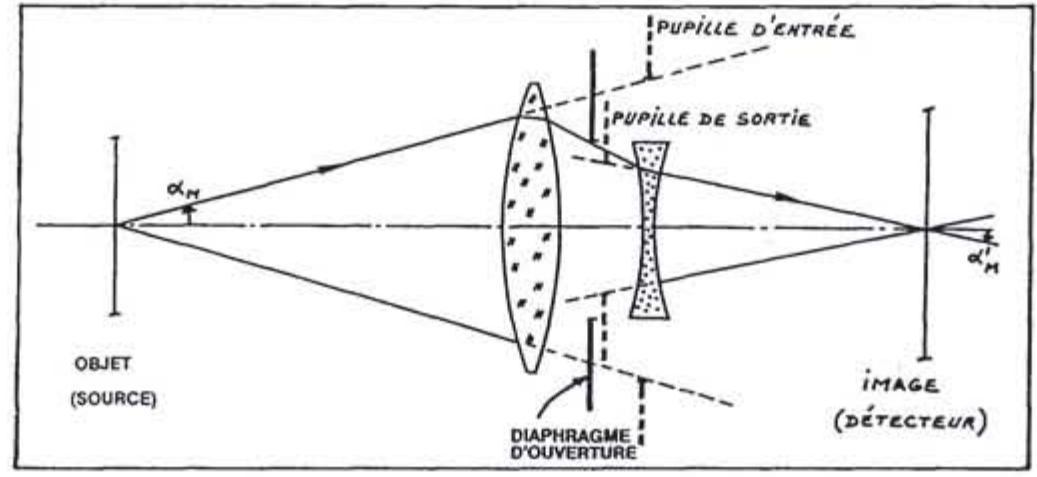

Fig 1-13 : Diaphragmes d'un système optique

Systèmes optiques aplanétlques

Dans de nombreuses applications telles que limagerie, le système optique est conçu de façon à reproduire au mieux dans l'image la configuration géométrique de l'objet initial.

On rappelle qu'un système optique est dit stigmatique pour 2 points $A$ et $A^{\prime}$ si du point source $A$, il donne une image ponctuelle $A^{\prime}$. Si ce point A est situé sur l'axe optique et si, de plus, le système est stigmatique pour l'ensemble des points proches de $A$ et situés dans le plan perpendiculaire à laxe passant par $A$, le système est dit "aplanétique".

Si un objet circulaire de rayon y situé dans ce plan illumine la pupille d'entrée du système avec un faisceau de demi-angle au sommet oum, l'étendue géométrique de ce faisceau dans le milieu objet est (d'après le paragrahpe 1.1.5) :

$$
\mathrm{G}=\pi \mathrm{A}_{\mathrm{obj}} \sin ^{2} \alpha_{\mathrm{M}}=\left[\pi \mathrm{y} \sin \alpha_{\mathrm{M}}\right]^{2}
$$

La conservation de l'étendue optique à la réfraction (cf paragraphe 1.3.1) permet d'écrire que :

$$
n^{2} G=n^{2} G^{\prime} \Rightarrow n y \sin \alpha M=n^{\prime} y \sin \alpha^{\prime} M
$$




\subsubsection{PHOTOMETRIE DES COLLECTEURS DE FLUX}

La géométrie d'un collecteur de flux est illustrée sur la Figure 1-15, où P'on constate que le flux émis par l'ensemble de la source et pénétrant dans la pupille d'entrée du système est capté par le détecteur à la transmission optique du système près.

Calcul du flux $F_{\boldsymbol{R}}$ Incident sur le détecteur :

La procédure la plus simple et la plus usitée, pour évaluer le flux capté FR, est de calculer, dans l'espace objet (celui comprenant la source et la pupille d'entrée du système) le flux émis par la source à lintérieur de la pupille d'entrée, puis de prendre en compte la transmission optique du système.

Si $\mathcal{T}$ et $\tau_{o p}$ sont les facteurs de transmission du milieu de propagation et de l'optique, I l'intensité de la source et $\Omega$ s l'angle solide sous lequel est vue la pupille d'entrée depuis la source $\left(\Omega S=S_{o p} / d^{2}\right)$, il vient :

$$
F_{R}=\tau\left|\Omega S \tau_{o p}=\tau\right| \tau_{o p} S_{o p} / d^{2}
$$

SI la source est caractérisée par son aire As et sa luminance Ls et l'ouverture du faisceau par l'angle aM dans l'espace objet, it vient :

$$
F_{R}=\pi \tau \text { Ls As } \tau_{o p} \sin ^{2} \alpha M
$$

\section{Eclairement du détecteur}

Dans le cas où la source est quasi ponctuelle (cas d'un laser monomode ou d'une source de dimensions finies à très grande distance), les dimensions et la forme de l'image (appelée la "tache image") sont déterminées par la conception optique du système (qualité limitée par la diffraction, influence des aberrations et défauts de mise au point, des inhomogénéités d'indice du milieu de propagation, etc...) Cette tache image étant de toute façon plus petite que le détecteur, l'éclairement de ce dernier n'est pas uniforme sur toute sa surface. Le système optique doit être caractérisé par un paramètre représentatif de la tache image obtenue dans la conjugaison objet image envisagée. Ce paramètre, dénommé "Réponse Impulsionnelle Spatiale" RIS (ou réponse percussionnelle ou Point Sprez.d Function PSF) représente le rapport entre la valeur de l'éclairement en tout point du plan image de coordonnées ( $\left.x^{\prime}, y^{\prime}\right)$ et sa valeur maximale :

$$
\text { RIS }\left(x^{\prime}, y^{\prime}\right)=E^{\prime}\left(x^{\prime}, y^{\prime}\right) ; E_{\max }^{\prime}
$$

Cette fonction bidimensionnelle est indépendante de la source dans la mesure où cette dernière est quasi ponctuelle et ne dépend que de la configuration optique du système. Elle se détermine en général par le calcul au niveau de la conception du système optique et peut se mesurer (après la réalisation) sur un banc muni de source quasi ponctuelle (collimateur).

La valeur de l'éclairement maximal dans le plan du détecteur, en présence de la source réelle, se déduit en écrivant que le flux total reçu par le détecteur est égal à l'intégrale de l'éclairement dans le plan image :

$$
\begin{aligned}
\mathrm{F}_{\mathrm{R}}=\tau I \Omega S & =\iint_{\text {planimage }} E(x, y) \cdot d x d y \\
& =\mathrm{E}_{\max } \iint_{\text {planimage }} R I S(x, y) d x d y
\end{aligned}
$$

D'où la valeur de l'éclairement local $E^{\prime}\left(x^{\prime}, y^{\prime}\right)$ en chaque point du détecteur :

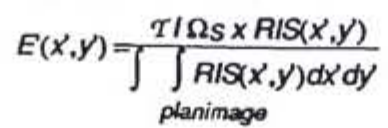


Cette relation dite "relation des sinus d'Abbe", lie les valeurs du champ et de l'ouverture ( $y^{\prime}$ et $\left.\alpha^{\prime} M\right)$ dans l'espace image à celles de l'espace objet dans le cas de systèmes aplanétiques.

Si R et $R^{\prime}$ sont les rayons respectifs des pupilles d'entrée et de sortie du système optique considéré. l'étendue optique du faisceau transmis par le système peut s'écrire, dans les espaces objet et image :

$$
n^{2} G=(\pi n R \sin \theta M)^{2} \quad n^{2} G^{\prime}=\left(\pi n^{\prime} R^{\prime} \sin \theta^{\prime} M\right)^{2}
$$

Les paramètres de champ et d'ouverture d'un système optique aplanétique sont ainsi reliés entre eux de la façon suivante: nysin $\alpha M=n R \sin \theta M=n^{\prime} R^{\prime} \sin \theta^{\prime} M=n^{\prime} y^{\prime} \sin \alpha^{\prime} M$

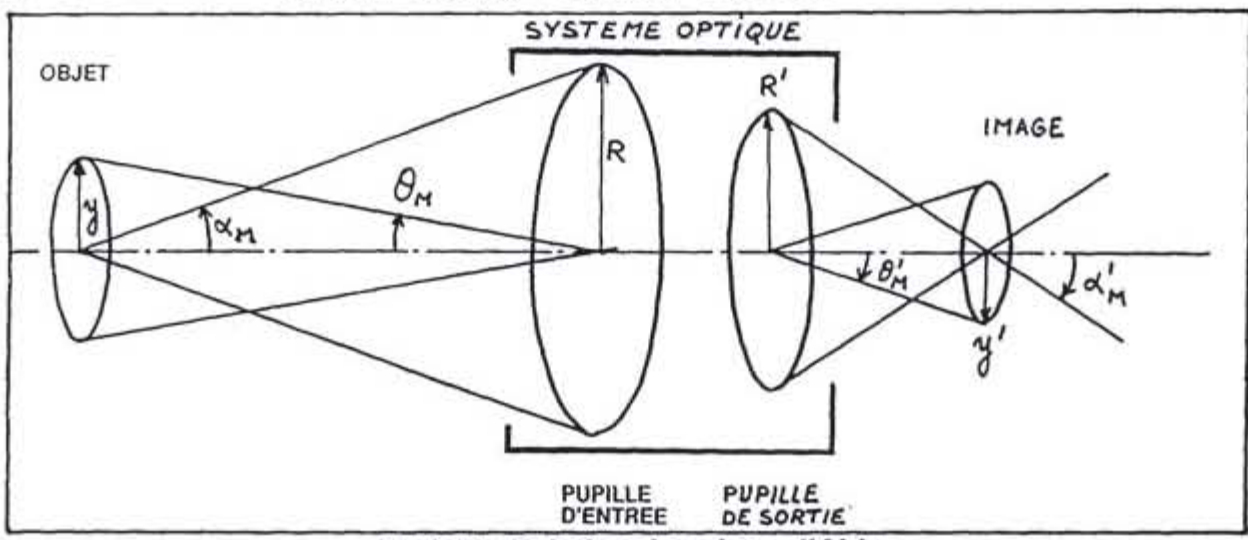

Fig l-14: Relation des sinus d'Abbe

Collecteurs de flux / Capteurs d'Images

En fonction de la nature de l'information recherchée sur un objet, on distingue traditionnellement 2 types de systèmes optiques : les collecteurs de flux et les capteurs d'images.

Les premiers sont destinés à capter le maximum du rayonnement émis par la source dans son ensemble. Pour cela, le champ en réception du système est surdimensionné par rapport aux dimensions (métriques ou angulaires, suivant le cas) de l'objet. On dit alors que l'objet n'est pas "résolu" par le système optique. dont il est le diaphragme de champ.

Comme son nom lindique, un capteur d'image est censé transposer point par point l'information optique de la scène dans son espace image, en général sur une surface, plane ou non, (plan de la pellicule d'un appareil photographique; du senseur d'une caméra de télévision, rétine de l'oeil). On dit alors que l'objet, ou la scène , est résolu par le système en un certain nombre "d'éléments de résolution" (picture elements ou "pixels" en anglais) indépendants les uns des autres et définis par les caractéristiques géométriques du détecteur associé à l'optique.

\section{Exemple :}

Calculer l'éclairement du détecteur dans un système optique limité par la diffraction observant une source quasi monochromatique, à la longueur d'onde $\lambda$, d'intensité I, à la distance d La pupille d'entrée du système a un diamètre $D$ et sa distance focale image est $f^{\prime}<<$. 
La qualité optique du système étant limité par la diffraction, la tache image est une figure d'Airy et la RIS, de révolution autour du point central peut s'écrire:

$$
R I S(n)=\left|\frac{2 \sqrt{1}\left(\pi D r / \lambda f^{\prime}\right.}{\pi D r / f^{\prime}}\right|^{2}
$$

où $J_{1}$ est la fonction de Bessel du ter ordre

où r est la distance d'un point du détecteur au centre de la tache. Sans entrer dans le détail des calculs, on peut montrer que l'éclairement maximal dans l'image est le suivant :

$$
E_{\max }^{\prime} \sim \pi \tau \mid \tau_{\infty} D^{4} / 4 f^{2} \lambda^{2} d^{2}
$$

et l'éclairement local en chaque point donné par la loi de variation de la figure d'Airy

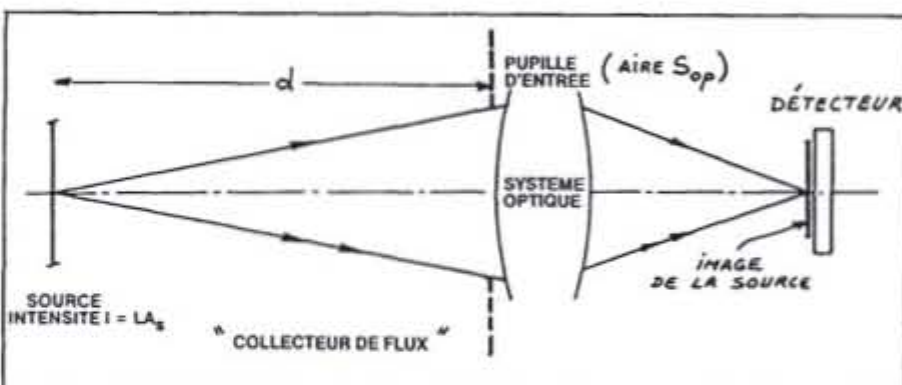

Fig 1-15: Géométrie d'un collecteur de flux

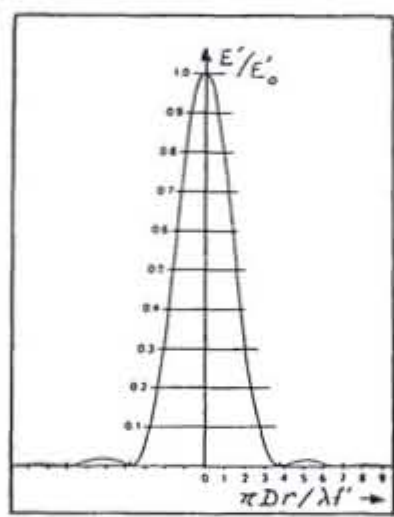

Fig l-16 : Eclairement dans le plan image d'un collecteurde flux limité par la diffraction

\subsubsection{PHOTOMETRIE DES CAPTEURS D'IMAGES}

Dans cette configuration, le système optique possède un ou plusieurs détecteurs. Les dimensions angulaires ou métriques de l'objet (ou source) sont supérieures au champ élémentaire (ou résolution) du système, défini par les dimensions des détecteurs, individuels et la distance focale de l'optique (pour une observation à l'infini). Chaque détecteur ne reçoit que le flux provenant de la zone dont il est l'image, les zones voisines étant imagées ailleurs.

Le flux incident sur chaque détecteur individuel est proportionnel à la luminance apparente du rayonnement de la source dans la zone utile et à l'étendue géométrique GR du capteur optique :

$$
F_{R}=L_{R} G_{R} \text { avec } L_{R}=L_{S} \tau_{T} \tau_{\infty}
$$


Calcul de l'étendue géométrique $\mathrm{G}_{\mathrm{R}}$ :

Le calcul de l'étendue géométrique $G_{R}$ du capteur optique peut se faire au choix dans l'espace objet. intermédiaire ou image du système.

Dans l'espace objet, rétendue géométrique du capteur optique est le produit de raire de sa pupille đ'entrée par rangle solide de réception sous tendu par chaque détecteur individuel, soit :

$$
\mathrm{G}_{\mathrm{R}}=\mathrm{S}_{\mathrm{op}} \Omega_{\mathrm{R}}=S_{o \rho}\left[\frac{A d}{F^{2}}\right] \quad \text { pour un objet à grande distance } d
$$

Le champ en réception de chaque détecteur définit sur l'objet une zone utile d’aire As telle que :

$$
A_{S}=A_{d} \times(1 / g y)^{2}=A d\left(d / t^{\prime}\right)^{2}
$$

oủ gy est le grandissement transversal du système optique.

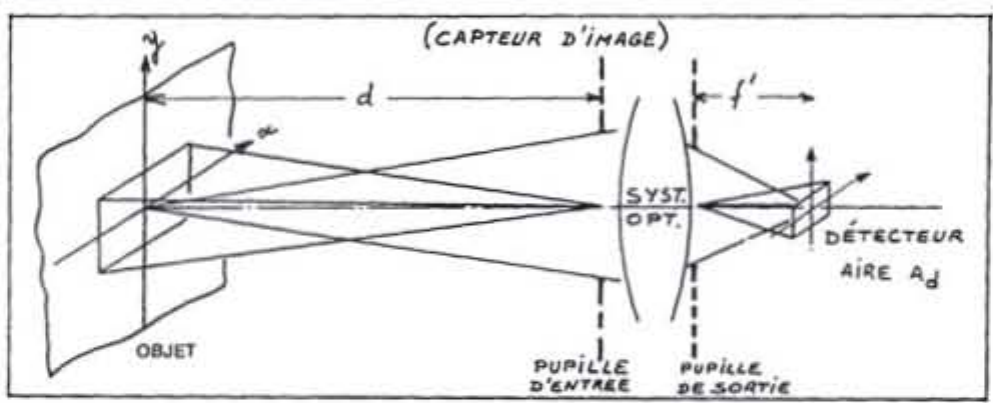

Fig 1 -17: Configuration d'un capteur d'image

Calcul de l'étendue géométrique dans l'espace Image

D'après ce qui a été vu précédemment (cf paragraphe I.1.6), l'étendue géométrique définie par le système optique et chacun de ses détecteurs individuels, d'aire Ad, est égale à :

$$
\mathrm{G}_{\mathrm{R}}=\pi \mathrm{Add}_{\mathrm{d}} \sin ^{2} \alpha_{M}^{\prime}
$$

oủ $\alpha^{\prime} M$ est le rayon angulaire sous lequel est vue la pupille de sortie depuis chacun de ces détecteurs. Pour une conjugaison infini / plan focal image, on définit le nombre d'ouverture $\mathrm{N}$ du système par :

$$
N=1 / 2 \sin \alpha^{\prime} M=f^{\prime} D
$$

oủ $\mathrm{D}$ est le diamètre de la pupille d'entrée.

L'expression de l'étendue du système optique dans l'espace image devient alors (si l'image est dans le plan focal) :

$$
G_{R}=\pi A d / 4 N^{2}
$$

Calcul de l'éclalrement du détecteur

L'éclairement du détecteur correspondant à une zone objet de luminance Ls est égal à :

$$
\begin{aligned}
E^{\prime}=\mathrm{FR}_{\mathrm{A}} / \mathrm{d} & =\pi \tau \tau \text { Top LS sin2 } 2 \alpha^{\prime} \mathrm{M} & & \text { (cas général) } \\
& =\pi \tau \tau \text { Top Ls } / 4 \mathrm{~N}^{2} & & \text { (image dans le plan focal) }
\end{aligned}
$$


Si l'objet est une surface diffuse en réflexion lambertienne, de facteur de réfléxion diffuse Rd et d'éclairement $E$, on rappelle que sa luminance est égale à :

$$
L_{S}=R_{d} E / \pi
$$

l'éclairement du détecteur $E$ ' peut se calculer alors en fonction de l'éclairement de la scène $E$

$$
\begin{aligned}
E^{\prime} & =\tau \mathcal{R}_{d} \mathcal{T}_{\phi p} E \sin 2 \alpha^{\prime} M & & \text { (cas général) } \\
& =\tau \tau_{d} \mathcal{T}_{o p} E / 4 N^{2} & & \text { (image dans le plan focal) }
\end{aligned}
$$

Exemple : Limite de fonctionnement de camescope.

La limite en éclairement minimal du détecteur d'un camescope est $E^{\prime}$ min =

Jusqu'à quelle valeur en éclairement de scène fonctionne le camescope, pour des scènes de réflectance $10 \%$. Le nombre d'ouverture minimal du camescope est $N_{\min }=1,4$.

Si la transmittance atmosphérique et la transmission de l'optique sont très bonnes $\left(T=T_{o p}=1\right)$, la valeur limite en éclairement de scène est :

$$
E_{\min }=\frac{4 N^{2} E^{\prime}}{\tau R_{d} \tau_{o p}}=3 \text { lux }
$$

\subsubsection{CAS DES SYSTEMES A CHAMP LARGE}

Les considérations précédentes concemaient des observations d'objets ou de sources situés à proximité de l'axe du système optique. De plus en plus nombreux sont les systèmes à grand champ (par exemple, caméras de télévision, appareils photographiques, ...) pour lesquels les résultats des paragraphes ci-dessus sont modifiés en fonction de l'angle de champ considéré.

Soit un système aplanétique pour un couple de plans objet (de luminance uniforme $L$ ) et image. Isolons sur le plan objet des zones de même aire As et évaluons l'éclairement de leur ir lage en fonction de leur éloignement de l'axe, l'éclairement sur l'axe E'o étant pris cornme référence.

Puisque la luminance de l'objet est uniforme, la variation d'éclairement dans le plan image provient de celle de l'étendue géométrique du système en fonction de la position du point objet. L'étendue pour une zone de l'objet sur l'axe est:

$$
G_{0}=A s S_{o p} / d_{0}^{2}
$$

si do est la distance du plan objet à la pupille d'entrée du système, d'aire $S_{o p}$, pour une zone d'objet vue sous I'angle de champ $\theta$, cette étendue devient :

$$
G_{\theta}=\frac{(A S \cos \theta) \times\left(S_{o \rho} \cos \theta\right)}{\left(d_{0} / \cos \theta\right)^{2}}=G_{0} \cos ^{4} \theta
$$

II en résulte que l'éclairement dans le plan image décrit du centre vers le bord de champ suivant une loi, dite loi en cosinus ${ }^{4}$ :

$$
E_{\theta}=E_{0} \cos ^{4} \theta
$$

En fait, cette loi, déduite dans le cas d'une lentille simple, est rarement vérifiée dans les systèmes optiques réels, où la présence de vignettage accentue la chute de l'éclairement en bord de champ. Une façon d'éliminer ces variations d'éclairement est đ'utiliser un système optique à champ réduit couplé à un dispositif de balayage angulaire placé près de la pupille du système. 


\subsection{GRANDEURS SPECTRALES}

\subsubsection{POSITION DU PROBLEME}

Les 3 premiers paragraphes de ce chapitre avaient pour objet de caractériser les propriétés spatiales et angulaires des rayonnements optiques et de leurs capteurs, grâce aux méthodes de la photométrie géométrique : en suivant le cheminement des rayons lumineux dans le système (source + milieu de propagation + optique de réception), cette dernière permet de calculer les signaux optiques à exploiter (flux sur un détecteur, éclairement, ...)

Nulle part, il n'a été fait mention du domaine de fonctionnement en longueur d'onde du système optique. qui est l'un des choix essentiels dans la conception de tout système optique et c'est l'objet de ce paragraphe que de considérer les propriétés spectrales des rayonnements.

Si l'on se reporte au bloc diagramme de la Figure $0-1$, on constate, après examen, que tous les éléments de la chaine optique (émission, milieux et surfaces de propagation, composants optiques, détecteurs) présentent un comportement et des performances très variables suivant le domaine de longueur d'onde envisagé : ainsi, le milieu de propagation (tel que l'atmosphère) peut avoir une excellente transmission á certaines longueurs d'onde, être totalement opaque pour d'autres, une source donnée émettra l'essentiel de son flux dans un domaine limité ou au contraire très large en longueur d'onde.

Du point de vue photométrique, la conception d'un système optique s'appuie donc non seulement sur les lois de la photométrie géométrique, mais aussi sur l'analyse des propriétés spectrales de ses composants, dont on rappelle les principales caractéristiques ci-dessous.

\subsubsection{SPECTRE D'UN RAYONNEMENT}

Comme on le verra au chapitre II, toute source émet ur rayonnement avec une répartition énergétique en fonction de la longueur d'onde, ou "spectre" qui lui est propre et qui constitue donc une "empreinte" représentative du rayonnement. Le comportement du rayonnement d'une source donnée, en fonction de la longueur d'onde peut se calculer théoriquement à partir des paramètres propres de la source (composition chimique, état solide liquide, gazeux, mode d'émission, température, etc...) ou se mesurer au moyen d'appareils qui décomposent ce rayonnement et en extraient les contributions à chaque longueur d'onde (spectroradiomètres).

Les propriétés spectrales d'un rayonnement se caractérisent par un ensemble de grandeurs dites "spectriques" (telles que flux, intensité, luminance, éclairement spectriques, ...), identiques aux grandeurs de la photométrie géométrique définies plus haut (paragraphe 1.1), mais considérées par unité de longueur d'onde autour d'une valeur de longueur d'onde donnée : si g représente la grandeur photométrique (telle que F, I, L,E, etc...) d'un rayonnement dont une contribution élémentaire dg provient du domaine spectral compris entre les longueurs d'onde $\lambda$ et $\lambda+d \lambda$, on dira que ce rayonnement possède, à la longueur d'onde $\lambda$, une grandeur spectrique $\left[\frac{d g}{d \lambda}\right]$. telle que :

$$
d g=\left[\frac{d g}{d a}\right] d a
$$

On parlera ainsi de flux spectrique $\left[\frac{d F}{d a}\right]$, luminance spectrique $d U d \lambda$, intensité spectrique $\left[\frac{d l}{d a}\right]$, etc... 
Les unités les plus généralement utilisées pour désigner une grandeur spectrique sont les unités de la grandeur photométrique correspondante rapportées à l'unité de longueur d'onde (le micromètre) : voir le tableau I-3 pour la correspondance entre grandeurs photométriques et spectriques.

Nota : Pour certaines applications particulières (spectroscopie par exemple), on spécifie les grandeurs spectriques non pas par unité de longueur d'onde, mais par unité de fréquence (Hertz). La transposition de l'une à l'autre se fait de la façon suivante:

La largeur de bande, en $\mu \mathrm{m}$, correspondant à̀ $1 \mathrm{~Hz}$ est :

$$
\begin{aligned}
& \lambda=c / v \\
& \lambda^{2}
\end{aligned} \quad \Rightarrow|d v|=\frac{c}{\lambda^{2}} d \lambda
$$

$$
\mathrm{d} \lambda=\lambda^{2} / \mathrm{c} \quad\left(0,3310^{-14} \mu \mathrm{m} \quad \text { à } \lambda=1 \mu \mathrm{m}\right)
$$

\begin{tabular}{|c|c|c|c|}
\hline $\begin{array}{c}\text { Grandeur } \\
\text { photométrique }\end{array}$ & $\begin{array}{l}\text { Grandeur } \\
\text { spectrique }\end{array}$ & Notation & Unité \\
\hline $\begin{array}{l}\text { FLUX } \\
F(W)\end{array}$ & $\begin{array}{c}\text { Flux } \\
\text { spectrique }\end{array}$ & $\frac{d F}{d \lambda}$ & $\mathrm{W} \mu \mathrm{m}^{-1}$ \\
\hline $\begin{array}{l}\text { INTENSITÉ } \\
\text { I }\left(\mathrm{W} \mathrm{sr}^{-1}\right)\end{array}$ & $\begin{array}{l}\text { Intensité } \\
\text { spectrique }\end{array}$ & $\frac{d l}{d \lambda}$ & $\mathrm{W} s r^{-1} \mu \mathrm{m}^{-1}$ \\
\hline $\begin{array}{l}\text { LUMINANCE } \\
\mathrm{L}\left(\mathrm{W} \mathrm{m} \mathrm{m}^{-2} \mathrm{sr}^{-1}\right)\end{array}$ & $\begin{array}{l}\text { Luminance } \\
\text { spectrique }\end{array}$ & $\frac{d L}{d \lambda}$ & $\mathrm{W} \mathrm{m} \mathrm{m}^{-2} \mathrm{sr}^{-1} \mu \mathrm{m}^{-1}$ \\
\hline $\begin{array}{l}\text { ECLAIREMENT } \\
\text { E }\left(\mathrm{W} \mathrm{m} \mathrm{m}^{-2}\right)\end{array}$ & $\begin{array}{l}\text { Eclairement } \\
\text { spectrique }\end{array}$ & $\frac{d E}{d \lambda}$ & $W m^{-2} \mu m^{-1}$ \\
\hline
\end{tabular}

Tableau 1-3: Correspondance entre grandeurs photométriques et spectriques.

Le calcul d'une grandeur photométrique g sur un domaine spectral fini s'effectue à partir de la grandeur spectrique correspondante, en sommant sur l'ensemble du domaine spectral considéré les contributions photométriques élémentaires provenant de chaque région du domaine et définies plus haut :

$$
d g=\left[\frac{d g}{d \lambda}\right] d \lambda
$$

La grandeur photométrique g sur le domaine de longueurs d'onde $\left(\lambda_{1}, \lambda_{2}\right)$ s'exprime donc par:

$$
g\left[\lambda_{1}, \lambda_{2}\right]=\int_{\lambda_{1}}^{\lambda_{2}}\left[\frac{d g}{d \lambda}\right] d \lambda
$$

Les grandeurs photométriques caractéristiques d'un rayonnement donné (flux, luminance, intensité,...) étant reliées entre elles par des lois géométriques, les grandeurs spectriques de ce rayonnement présentent la même loi de variation relative en fonction de la longueur d'onde :

$$
\frac{d F / d}{(d / d d)_{\max }}=\frac{d / d \lambda}{(d / d \lambda)_{\max }}=\frac{d / d \alpha}{(d / d d)_{\max }}=S \lambda
$$

Cette loi, normée et sans dimension, est caractéristique de l'évolution relative du spectre, mais ne donne pas de renseignements sur la valeur absolue des grandeurs spectriques définies ci-dessus. Elle permet de repérer les domaines de longueur d'onde où le rayonnement est concentré. Elle se dénomme "répartition spectrale relative" ou "composition sfectrale relative" du rayonnement. 


\subsubsection{CARACTERISATION SPECTRALE DES SURFACES ET MILIEUX DE PROPAGATION}

Comme les sources de rayonnement elles-mêmes, les composants optiques, surfaces et milieux de propagation, situés le long du parcours des photons se comportent différemment d'une longueur d'onde à une autre, suivant leur composition chimique, concentration, état de surface, etc... Chacun d'eux imprime donc son "empreinte spectrale" sur le faisceau lumineux et entraine une modification spectrale plus ou moins importante du rayonnement en sortie.

L'action d'un composant optique sur un rayonnement se traduit par des phénomènes tels que réflexion, transmission (de flux ou de luminance), atténuation, absorption, diffusion, turbulence, ... , qui peuvent en modifier la configuration et/ou les grandeurs photométriques et se caractérise au moyen de paramètres destinés à quantifier leur performance. Ces paramètres, généralement appelés "facteurs de" (nom du phénomène : réflexion, transmission, ...) sont définis par le rapport de 2 grandeurs photométriques (identiques ou non) carcactérisant le rayonnement avant et après son passage dans le composant.

Par exemple, le facteur de transmission géométrique (ou régulière) d'une lame à faces parallèles pour une incidence de rayonnement donnée est le rapport entre les valeurs de la luminance du rayonnement à la sortie et à l'entrée.

La plupart des composants optiques dans la chaîne ayant (sauf dans certains cas particuliers) un comportement différent en fonction de la longeur d'onde et de la géométrie (incidence, divergence, etc...) du faisceau incident, il est très souvent nécessaire de les caractériser expérimentalement au moyen de rayonnements dits simples (pinceaux de lumière quasi collimatés et quasi monochromatiques) dont on peut faire varier les paramètres géométriques (angle d'incidence) et spectraux (longueur d'onde) de façon indépendante.

Ainsi, un composant en réflexion sera-t-il caractérisé par son facteur de réflexion spectrale, défini pour une incidence de rayonnement donné.

On distinguera :

- le facteur de réflexion spectrale régulière ou géométrique $\mathcal{R}_{g}(\lambda)$, égal au iapport entre les valeurs de la luminance spectrique du rayonnement après et avant réflexion (mesurée dans la direction de l'optique géométrique):

$$
\left[\frac{d L}{d a}\right]_{r}=R_{g}(\lambda)\left[\frac{d L}{d \lambda}\right]_{i}
$$

- le facteur de réflexion totale $\mathcal{R}_{1}(\Omega)$ spectrale égal au rapport entre les valeurs de flux spectrique après et avant réflexion :

$$
\left[\frac{d F}{d a}\right]_{r}=R_{t}(\lambda)\left[\frac{d F}{d \lambda}\right]_{i}
$$

- le facteur de réflexion spectrale $R_{d}(\lambda)$ diffuse d'une surface lambertienne, telle que :

$$
\left[\frac{d L}{d \lambda}\right]_{r}=\frac{R(\lambda)}{\pi}\left[\frac{d E}{d \lambda}\right]_{i}
$$

Les quantités affectées de l'indice $r$ se rapportent au rayonnement réfléchi par le composant. Celles affectées de l'indice i se rapportent au rayonnement incident. 


\subsubsection{TRANSMISSION SPECTRALE D'UN MILIEU DE PROPAGATION}

L'un des paramètres importants dans la conception d'un système optique, en particulier, dans le cas où le capteur est éloigné de la source, est la transmission spectrale du milieu de propagation, qui peut être affectée principalement par l'absorption et la diffusion du rayonnement par ce milieu, sources principales d'interactions entre la lumière et la matière.

\section{Cas de l'absorption}

L'absorption de rayonnement par un milieu donné consiste en une interaction entre les photons du rayonnement et les atomes ou molécules du milieu, qui résulte en chaleur (élévation de température) et disparition du photon incident.

Pour évaluer linfluence de l'absorption du milieu sur la propagation d'un rayonnement, considérons un pinceau lumineux quasi monochromatique $(\lambda)$ se propageant dans le milieu le long d'un axe $x$. A cause des propriétés absorbantes du milieu, le nombre de photons utiles au rayonnement se réduit tout au long du parcours pour participer à l'échauffement (plus ou moins important) du milieu : ainsi le flux $F(\lambda, x+d x)$ mesuré à une abscisse $(x+d x)$ est-il légèrement réduit par rapport à la valeur qưil avait encore à l'abscisse $x$ :

$$
F(\lambda, x+d x)=F(\lambda, x)-d F_{a}(\lambda, x)
$$

d'une quantité $\mathrm{dF}_{\mathrm{a}}(\lambda, \mathrm{x})$ égale au flux absorbé par le milieu dans la tranche d'épaisseur $\mathrm{dx}$.

On considère que le flux absorbé est proportionnel à la valeur du flux $F(\lambda, x)$ à l'entrée de cette tranche, à l'épaisseur dx et à un paramètre spectral représentatif de l'absorption du milieu à cette longueur d'onde et à l'endroit considéré, appelé coefficient d'absorption linéique spectral, $\alpha(\lambda, x)$ :

$$
\mathrm{dF}_{\mathrm{a}}(\lambda, \mathrm{x})=\alpha(\lambda, \mathrm{x}) \mathrm{F}(\lambda, \mathrm{x}) \mathrm{dx}
$$

II ressort des 2 équations ci-dessus que la valeur du flux $F(\lambda, x)$ après un parcours de longueur $x$ dans le milieu est par rapport à sa valeur initiale $F(\lambda, 0)$ ézale à :

$$
F(\lambda, x)=F(\lambda, 0) \exp \left[-\int_{0}^{x} \alpha(\lambda, x) d x^{\prime}\right]
$$

La transmission spectrale du milieu due à l'absorption sur une distance $\mathrm{x}$ est alors :

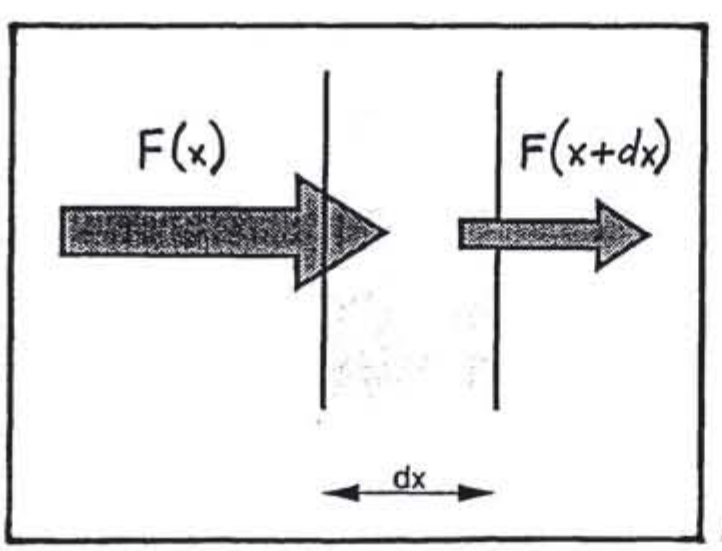

$$
\mathcal{T}_{a}(\lambda, x)=F(\lambda, x) / F(\lambda, 0)=\exp \left[-\int_{0}^{x} \alpha(\lambda, x) d x^{\prime}\right]
$$

Dans le cas où le milieu de propagation est dit homogène, c'est-à-dire, si $\alpha(\lambda, x)$ est indépendant de $x$, le flux propagé par le rayonnement quasi monochromatique décrit exponentiellement en fonction de la distance parcourve :

$$
\begin{aligned}
& F\left(\lambda_{1} x\right)=F(\lambda, 0) \exp [-\alpha(\lambda) x] \\
& \mathcal{T}_{e}\left(\lambda_{2} x\right)=\exp [-\alpha(\lambda) x]
\end{aligned}
$$

Fig 1-18: Absorption de rayonnement par le millieu de propagation 


\section{Cas de la diffusion}

La diffusion d'un rayonnement par un milieu optique consiste en une interaction entre une fraction des photons de ce rayonnement et les atomes et/ou molécules du milieu, qui résulte en une redistribution angulaire de cette fraction de rayonnement (avec modification ou non de la longueur d'onde).

La procédure généralement suivie pour révaluation en transmission d'un milieu de propagation diffusant est semblable à celle définie ci-dessus pour le traitement de l'absorption. On caractérise dans ce cas le milieu par son coefficient de diffusion linéique spectrale $\beta(\lambda, x)$ tel que le flux recueilli à labscisse $x+d x$ et se propageant dans la direction du flux initial est :

$$
\begin{aligned}
& F(\lambda, x+d x)=F(\lambda, x)-d F_{d}(\lambda, x) \\
& \text { avec dFd }(\lambda, x)=\beta((\lambda, x) F(\lambda, x) d x
\end{aligned}
$$

La grande différence entre absorption et diffusion provient du fait que le flux diffusé ne disparaît pas localement comme le flux absorbé : il est réémis par le milieu à une longueur d'onde identique (diffusions de Rayleigh, de Mie) ou différente (diffusion Raman).

II est parfois difficile de distinguer les photons transmis régulièrement (ou géométriquement) de ceux qui après diffusion, suivent un parcours très semblable.

La caractérisation d'un milieu en diffusion entraîne la mesure de l'indicatrice de diffusion, indicatrice en intensité de la lumière diffusée par unité de volume du milieu.

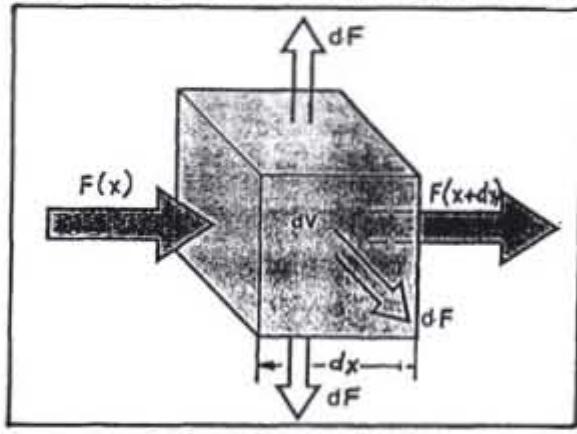

Phénomène de diffusion

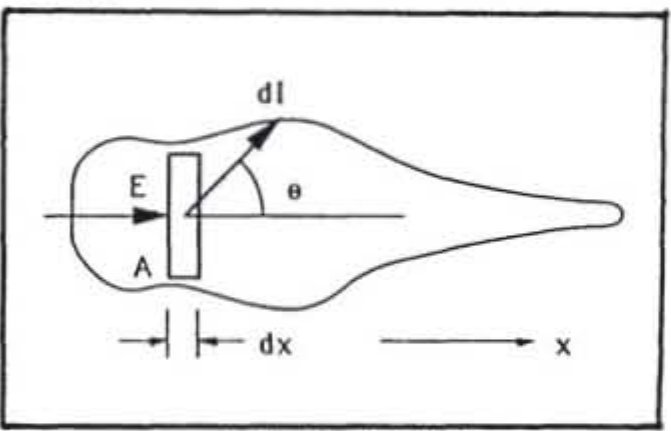

Indicatrice de diffusion

Fig 1-19 : Diffusion par le milieu de propagation

De même que pour l'absorption, on définit une transmission (dite géométrique) du milieu en diffusion

$$
\begin{aligned}
\tau_{d}(\lambda, x) & =\exp \left[\int_{0}^{x} \beta(\lambda, x) d x\right] \\
& =\exp [-\beta(\lambda) x]
\end{aligned}
$$

(milieu homogène)

Transmission spectrale globale d'un milleu

Si le milieu de propagation présente à la fois de l'absorption et de la diffusion, la transmission régulière (ou géométrique) du milieu sur un parcours de longueur $x$, c'est-à-dire mesurée dans l'étendue géométrique du pinceau initial) peut alors s'exprimer en fonction du coefficient $\alpha(\lambda, x)$ tel que :

$$
\sigma(\lambda, x) i=\alpha(\lambda, x)+\beta((\lambda, x)
$$

appelé coefficient d'extinction spectrale linéique du milieu considéré. II vient alors :

$$
\begin{aligned}
& \tau(\lambda, x)=\exp \left[-\int_{0}^{x} \sigma\left(\lambda, x^{\prime}\right) d x^{\prime}\right] \\
& \tau(\lambda, x)=\exp [-\sigma(\lambda) x]
\end{aligned}
$$

(milieu inhornogène)

(milieu homogène) 
On rencontre dans la littérature (articles scientifiques, fiches techniques de composants) plusieurs façons d'exprimer la transmission des matériaux optiques ou milieux de propagation. Les termes principaux avec leur correspondance mathématique (pour matériaux homogènes) sont donnés dans le tableau ci-après :

Tableau 1-4: Caractérisation en transmission de milieux

\begin{tabular}{|c|c|c|}
\hline Dénomination & $\begin{array}{c}\text { Expression } \\
\text { Mathématique }\end{array}$ & $\begin{array}{l}\text { Transmission du } \\
\text { milieu sur distance } \mathrm{x}\end{array}$ \\
\hline $\begin{array}{c}\text { Transmission } \\
\text { spectrale par unité } \\
\text { de !ongueur }\end{array}$ & $\tau(\lambda)=e^{-\sigma(\lambda)}$ & $\tau(\lambda, x)=\tau^{X}(\lambda)$ \\
\hline $\begin{array}{l}\text { Densité optique } \\
\text { spectrale par unité } \\
\text { de longueur }\end{array}$ & $d(\lambda)=\log _{10} \tau(\lambda)=0,430(\lambda)$ & $T(\lambda, x)=10^{-d(\lambda) x}$ \\
\hline $\begin{array}{l}\text { Densité optique } \\
\text { spectrale }\end{array}$ & $D(\lambda)=\alpha(\lambda) x$ & $\tau(\lambda, x)=10^{-D(\lambda)}$ \\
\hline $\begin{array}{l}\text { Atténuation } \\
\text { spectrale par unité } \\
\text { de longueur (dB) }\end{array}$ & $\operatorname{att}(\lambda)=10 \log _{10} \frac{1}{\tau(\lambda)}$ & $\tau(\lambda, x)=10^{-\frac{\text { att }}{10} x}$ \\
\hline $\begin{array}{c}\text { Atténuation } \\
\text { spectrale (dB) }\end{array}$ & $\operatorname{ATT}(\lambda)=X . \operatorname{att}(\lambda)$ & $\tau(\lambda, x)=10 \frac{A T T(\lambda)}{10}$ \\
\hline
\end{tabular}

Les figures ci-dessous expriment les transmissions spectrales de l'atmosphère et d'une fibre optique pour illustrer ce paragraphe.

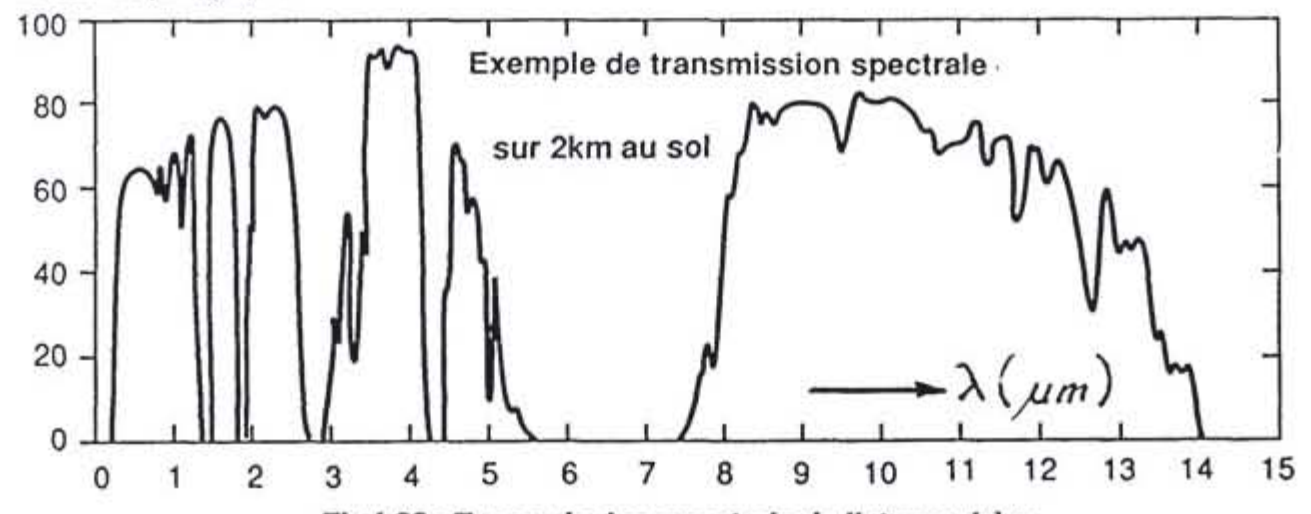

Fig 1-20: Transmission spectrale de l'atmosphère

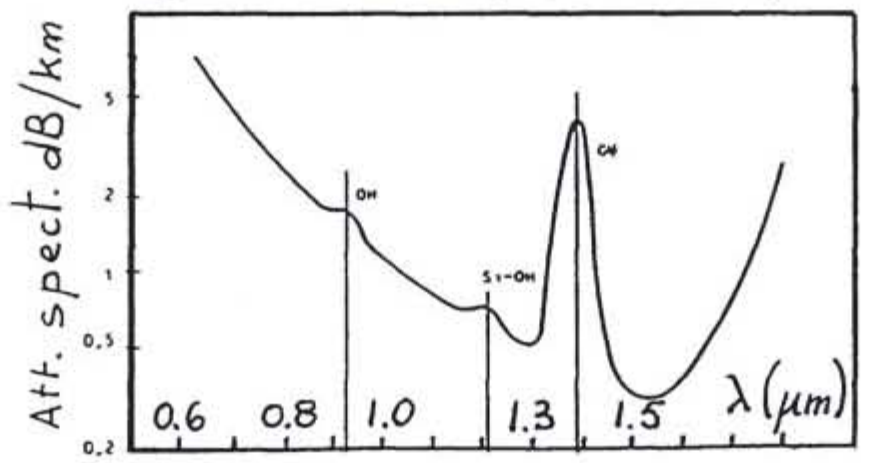

Fig 1-21: Transmission spectrale d'une fibre optique 


\section{CHAPITRE 2 \\ EMISSION DU RAYONNEMENT}

Dans tout système optique, la source de lumière constitue le premier maillon de la chaîne photométrique, dont elle détermine l'ensemble des caractéristiques. Le concepteur du système examinera donc avec grand soin les paramètres géométriques, spectraux et temporels des sources dont il dispose (imposées ou non par la mission du système), pour optimiser l'ensemble de l'instrument.

Le rayonnement optique le plus répandu provient de l'agitation des atomes et molécules en fonction de la température puisque tout corps à une température différente de $0 \mathrm{~K}$ énet un tel rayonnement, dit thermique ou par incandescence. Ces échanges d'énergie thermique sous forme de rayonnements participent à l'équilibre thermodynamique des corps, toute émission (ou perte d'énergie) étant compensée par l'absorption du rayonnement thermique ambiant.

La seconde origine de rayonnement optique, dite par luminescence, est liée à une excitation des atomes et molécules des corps par des moyens extérieurs, autres qu'une élévation de leur température.

Tout émetteur optique émet un rayonnement dont une fraction est caractéristique de sa température (rayonnement thermique) et une autre liée à sa composition, sa phase et le mode d'excitation de ses électrons (rayonnement par luminescence).

Ce chapitre présente les deux types d'émission, rappelle leur principe, décrit leurs propriétés essentielles dont il illustre les ordres de grandeur par quelques unes des sources les plus représentatives.

II.1. LE RAYONNEMENT THERMIQUE

\section{II.1.1. LOI DE KIRCHHOFF}

Soient 2 corps $X$ et $Y$, placés dans une enceinte isotherme à une température $T$, différente de $0 \mathrm{~K}$ (voir Fig II-1). A l'équilibre thermique, le rayonnement émis par chacun de ces corps constitue pour lui une perte d'énergie, compensée par l'absorption du rayonnement thermique ambiant. On peut démontrer que les échanges thermiques entre tout couple d'éléments de l'enceinte (par exemple entre un élément de $X$ et un de $Y$ ) doivent s'équilibrer dans chaque sens (c'est-à-dire de $X$ vers $Y$ et vice-versa) et ceci à toute longueur d'onde.

Si ces 2 corps sont placés dans le même milieu, supposé parfaitement transparent, le flux absorbé par un élément de $X$ (dont l'aire est $\Delta S_{x}$ ) et provenant d'un élément de $Y$ (d'aire $\Delta S_{y}$ ) dans la bande spectrale comprise entre les longueurs d'onde $\lambda$ et $\lambda+d \lambda$ est égal à :

$$
\lambda_{x}\left(\lambda, T, \theta_{x}, \varphi_{x}\right)\left[\frac{d L}{d \lambda}\right]_{y}^{T}\left(\theta_{y}, \varphi_{y}\right) \Delta G
$$

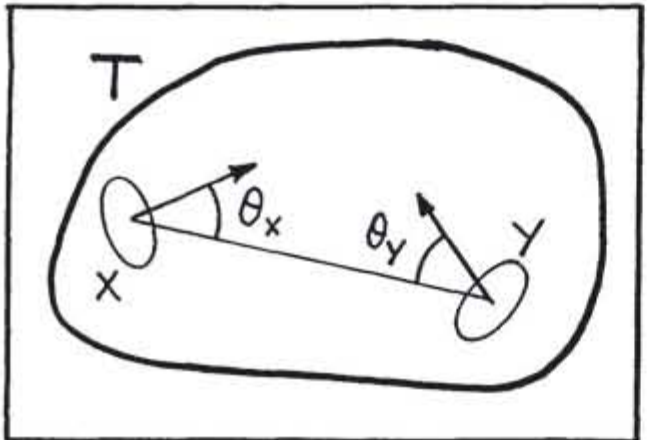

Fig $11-1$ : Echanges thermiques dans une enceinte Isotherme où $\lambda_{x}\left(\lambda, T, \theta_{x}, \phi_{x}\right)$ est le facteur d'absorption spectrale directionnelle du corps $X$ à la température $T$ et pour un flux incident sous l'angle défini par les valeurs $\left(\theta_{x}, \phi x\right)$.

$\left[\frac{d L}{d \lambda}\right]_{y}^{T}\left(\theta_{y}, \varphi_{y}\right)$ est la luminance spectrique du rayonnement thermique émis par le corps $Y$ dans la direction $\left(\theta_{y}, \phi_{y}\right)$ par rapport à la normale au corps $\mathrm{Y}$.

$\triangle G$ est rétendue géométrique définie par ces 2 éléments des corps $\mathrm{X}$ et $\mathrm{Y}$. 
Si l'on exprime de façon analogue le flux absorbé dans la mẻme bande spectrale par l'élément de $Y$ provenant du corps $\mathrm{X}$, on peut écrire:

$$
\frac{[\alpha d / d \lambda]_{x}^{T}\left(\theta_{x, \varphi x}\right)}{\lambda_{x}\left(\lambda, T, \theta_{x}, \varphi_{x}\right)}=\frac{[d y / \alpha]_{y}^{T}\left(\theta_{y}, \varphi y\right)}{\lambda_{y}\left(\lambda, T, \theta_{y}, \varphi_{y}\right)}
$$

Supposons maintenant que le corps $Y$ soit constitué d'un matériau idéal, absorbant totalement tout rayonnement incident, quelles que soient sa longueur d'onde et sa direction d'incidence $(\theta, \phi)$.

Un tel corps (ou surface) est dénomme "Corps Noir", parce qu'il apparait noir dans le visible, a température ambiante. Dans ces conditions, on peut écrire que :

$$
\begin{aligned}
\lambda_{y}\left(\lambda, T, \theta_{y}, \varphi_{y}\right) & =\lambda_{C}(\lambda, T, \theta C N, \varphi C N)=1 \\
d o \dot{u}:[\sigma / / a \alpha]_{x}^{T}\left(\theta_{x}, \varphi_{x}\right) & =\lambda_{x}\left(\lambda, T, \theta_{x}, \varphi_{x}\right)[\alpha / / \alpha]_{x}^{C N}(\theta C N, \varphi C N)
\end{aligned}
$$

En plaçant 2 Corps Noirs dans la même enceinte isotherme, on peut démontrer par ailleurs que le corps noir est une source lambertienne. II ressort donc de ces considérations thermodynamiques que la luminance spectrique d'une source thermique quelconque $X$, à une température $T$ et dans une direction d'émission définie par les angles $\theta$ et $\phi$, est proportionnelle au facteur d'absorption spectrale du matériau dont est constituée la source (mesuré à la même température et pour la même direction d'incidence) et à la luminance spectrique du Corps Noir à cette température :

$$
[d / / a]_{x}^{T}(\theta, \varphi)=\lambda_{x}(\lambda, T, \theta, \varphi)[d / d a]_{C N}^{T}
$$

Toute source thermique est caractérisée par son facteur d'émissivité spectrale directionnelle, rapport entre sa luminance spectrique et celle du corps noir à la même température. La loi de Kirchhoff ci-dessus montre que :

$$
\varepsilon(\lambda, T, \theta, \varphi)=\mu(\lambda, T, \theta, \varphi)
$$

Le comportement en émission d'un matériau est dicté par la valeur de son coefficient d'absorption. Pour cela, $A(\lambda, T, \theta, \phi)$ est aussi appelé "facteur d'absorption/émission spectrale" du matériau.

\section{II.1.2. PROPRIETES SPECTRALES DU RAYONNEMENT DU CORPS NOIR}

Le paragraphe précédent démontre limportance des propriétés en émission du Corps Noir, dont les caractéristiques servent de base à l'évaluation de tout rayonnement thermique réel et sont rappelées brièvement ci-dessous (avec les expressions mathématiques au tableau II-1) :

- le rayonnement du corps noir (ou absorbeur parfait : $Я(\lambda)=1$ ) est lambertien (sa luminance ne dépend pas de la direction d'émission) et non polarisé.

- sa luminance spectrique est définie à chaque température et chaque longueur d'onde par la loi de Planck

- son rayonnement spectral est maximal pour une valeur de la longueur d'onde inversement proportionnelle à la température (loi "du déplacement de Wien")

- son rayonnement global (sur tout le spectre) ne dépend que de sa température (loi de "Stefan") 
Tableau 11-1 : Expressions mathématiques des lois du Corps Noir :

\begin{tabular}{|c|c|c|}
\hline QUANTITE (loi) & Energétique & Photonique \\
\hline $\begin{array}{l}\text { Luminance } \\
\text { spectrique } \\
\text { (Planck) }\end{array}$ & $\begin{array}{c}{[d L e / d \Omega] E_{N}} \\
2 h c^{2} / \lambda^{5}\left[\frac{h c}{e \lambda k T}-1\right]\end{array}$ & $\begin{array}{c}{\left[d L_{\rho} / d \lambda\right]_{C N}^{T}} \\
2 c / \lambda^{4}\left[e \frac{h c}{e \lambda k T}-1\right]\end{array}$ \\
\hline $\begin{array}{l}\text { Coordonnées du } \\
\text { maximum d'émission } \\
\text { (déplacement de Wien) }\end{array}$ & $\begin{array}{c}\lambda_{m} T=K_{1}=2898 K \mu m \\
{[d L e / d \lambda]_{M}=K_{2} T^{5}}\end{array}$ & $\begin{array}{l}\lambda_{m} T=3670 \mu m K \\
\quad\left[d L_{\rho} / d \lambda\right] M=K_{2}^{\prime} T^{4}\end{array}$ \\
\hline Luminance totale (Stefan) & $L_{\theta}=K_{3} T^{4}$ & $L_{p}=\kappa_{3} T^{3}$ \\
\hline
\end{tabular}

La Figure suivante montre les courbes de luminance spectrique de Corps Noirs en fonction de la température :

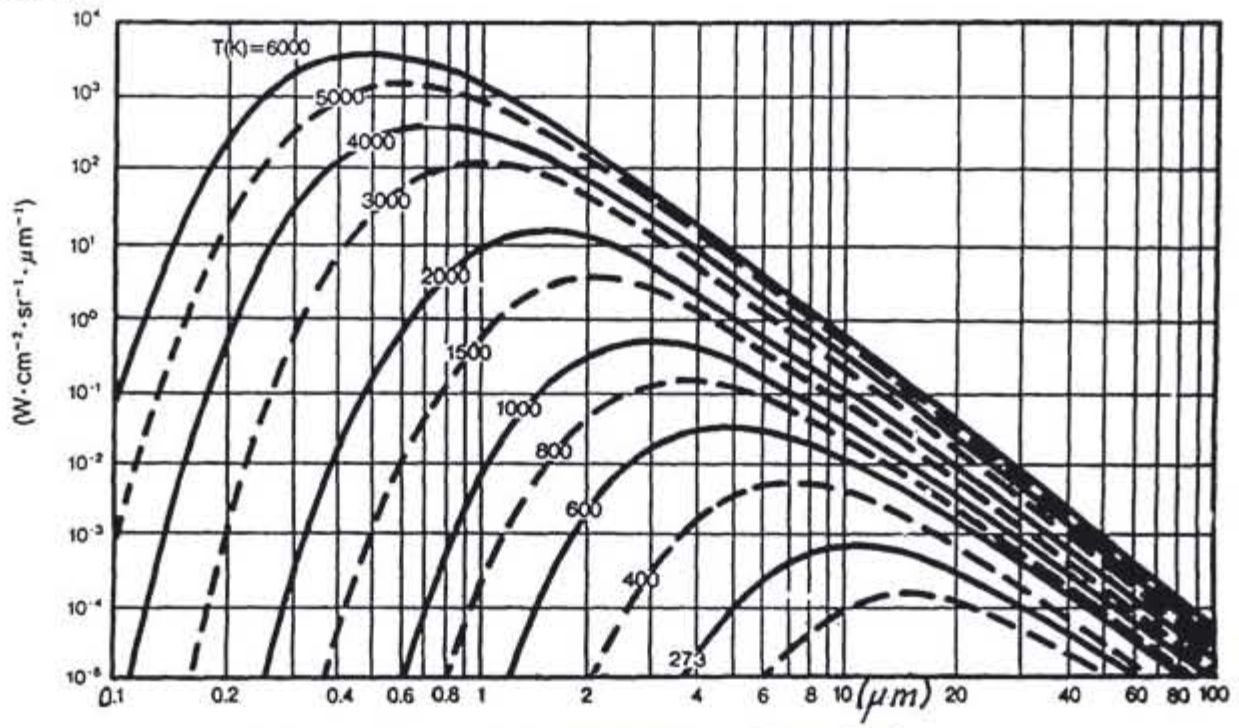

Fig II-2 : Luminance spectrique du Corps Noir (unités énergétiques)

La formule de Planck conduit à une représentation dite "universelle" de l'émission du Corps Noir, identique pour tous les Corps Noirs, indépendamment de leur température au moyen de 2 courbes $f$ et $\mathrm{g}$ représentatives respectivement : de la luminance spectrale relative :

$$
\begin{aligned}
& \left(\lambda \lambda_{m}\right)=f(x)=\left[d L_{e} / d \lambda\right] /\left[d L_{e} / d \lambda\right]_{\max } \\
& g(\lambda, \lambda m)=g(x)=\frac{\int_{0}^{\lambda}\left[d L_{0} / d \lambda\right]_{C N}^{T} d \lambda}{\int_{0}^{\infty}[d L e / d \lambda]_{C N}^{T} d \lambda} \quad x=\lambda \lambda_{m}
\end{aligned}
$$




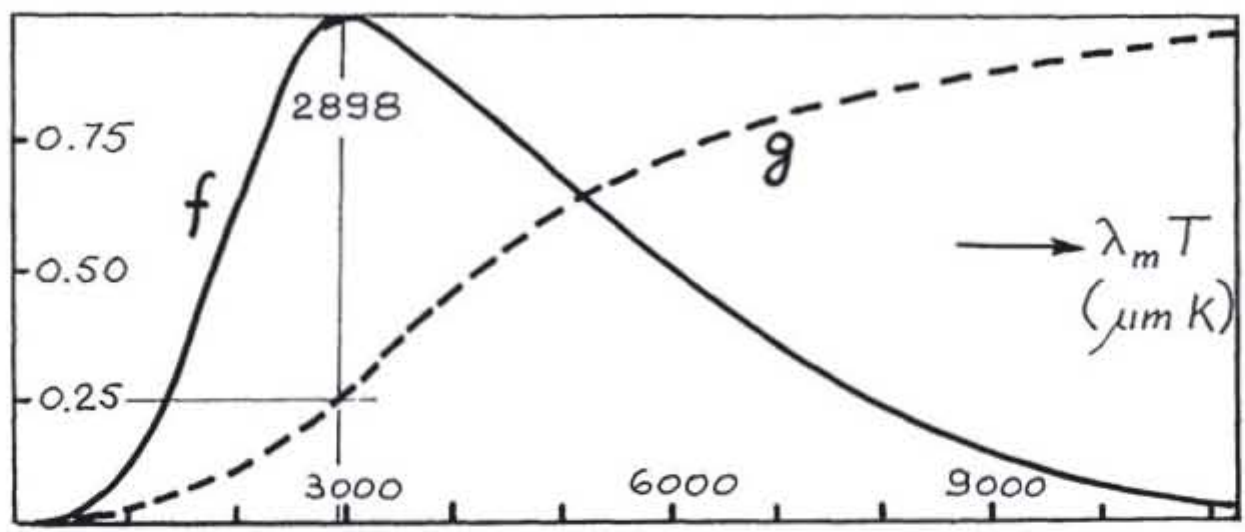

Fig $11-3$ : Formules "universelles" de rayonnement du Corps Noir

\section{II.1.3. EXEMPLES DE SOURCES THERMIQUES}

\section{Lampe à fliament de tungstene}

L'une des sources les plus uttisées en éclairage (éclairage domestique, automobile. ...) est la lampe á filament de tungstène. L'oeil humain étant sensible entre 0,4 et $0,7 \mu \mathrm{m}$, la source thermique la mieux adaptée aux applications visuelles telles que l'éclairage doit avoir son pic d’émission voisin de $\lambda=0,5 \mu \mathrm{m}$, ce qui, d'après la loi du déplacement de Wien correspond à une temperature de l'ordre de $6000 \mathrm{~K}$ (cas du soleil).

L'un des matériaux terrestres les plus réfractaires est le tungstène, qui, lorsqu'il est utlisé au plus près de sa température de fusion ( $T \mathrm{f}=3400 \mathrm{~K}$ ), présente un spectre d'émission assez riche dans le visible. Le graph \ de la Figure ci-dessous (Fig II-4a) montre l'évolution du facteur d'absorption-émission du tungstène en fonction de la longueur d'onde dans le visible et le proche infrarouge et celui de la Figure II-4b compare la luminance spectrique du tungstène (en direct ou au travers dune ampoule) avec celle du corps noir à la même température.

Les lampes à filament de tungstène les plus performantes ("lampes à halogènes") fonctionnent avec un filament à près de $3200 \mathrm{~K}$.
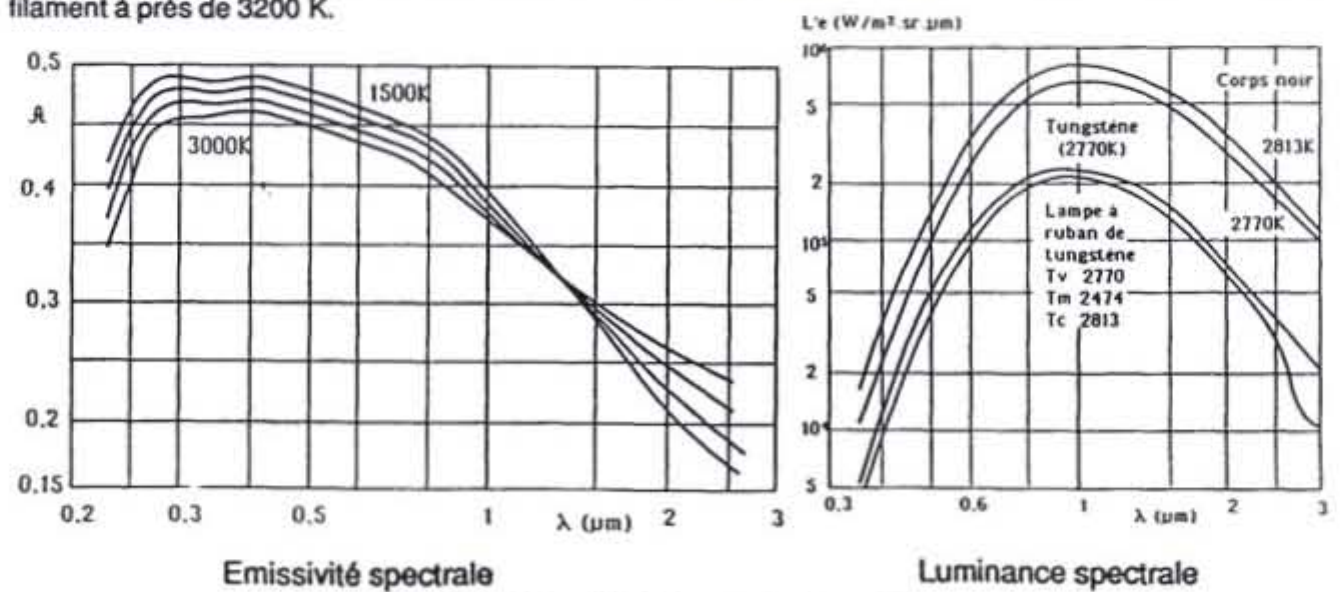

Fig II-4 : Caractéristiques du tungstène 
Le solell

Parmi les sources naturelles, le soleil foumit un rayonnement qui, dans son ensemble, peut être assimilé à celui d'un corps noir à la température de $5900 \mathrm{~K}$. Les courbes de la Figure II-5 représentent l'éclairement spectrique relatif dô au soleil, au niveau de la terre : la courbe "hors atmosphère" correspond à un éclairement global de l'ordre de $1450 \mathrm{~W} / \mathrm{m}^{2}$ et la courbe "au sol" à un éclairement global de l'ordre de $900 \mathrm{~W} / \mathrm{m}^{2}$ (voir la corrélation avec la courbe de transmission spectrale de l'atmosphère, paragraphe 1.4). Ces courbes montrent les contributions respectives de chaque "fenêtre atmosphérique" à l'éclairement du sol.

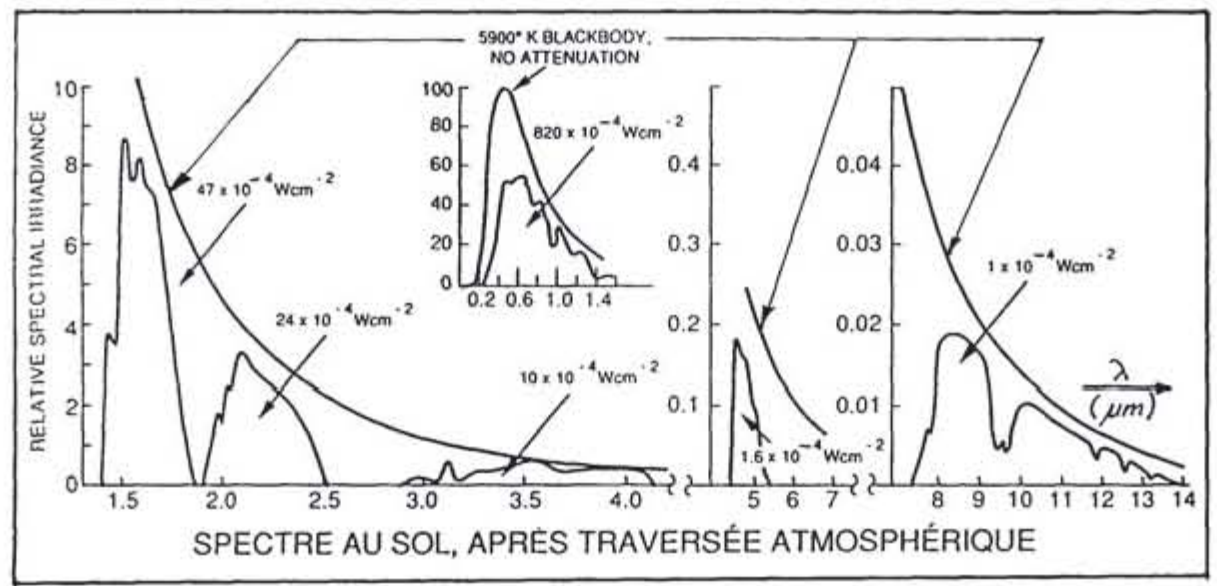

Fig II-5 : Eclairement spectrique relatif de la terre da au soleil

Exercice : Calcul de l'éclairement solaire hors atmosphère $(T$ SOLEIL $=5900 \mathrm{~K}$ )

L'éclairement solaire est égal à : $\quad E=L s \Omega s \quad$ ( $\Omega$ s angle solide du soleil vu de la terre)

D'après la loi de Stefan, la luminance totale du soleil (Corps Noir à $5900 \mathrm{~K}$ ) est égale à :

$$
L_{S}=K_{3} T S \Rightarrow E=K_{3} T_{S}^{A} \pi \alpha \xi=1450 \mathrm{~W} / \mathrm{m}^{2}
$$

\section{II.1.4. CAS DE SCENES NATURELLES}

Pour des applications telles que limagerie de scènes naturelles, il est important de connaître assez finement le comportement spectral des sources pour optimiser le fonctionnement du système optique á concevoir.

Les 2 contributions essentielles dans le rayonnement d'une source naturelle sont d'une part son émission thermique propre en fonction de sa température, et d'autre part, la réflexion ou diffusion de l'éclairement ambiant.

Dans le cas de surfaces diffusantes, lambertiennes, la luminance spectrique peut s'exprimer simplement de la façon suivante (avec les notations des paragraphes I.4.3 et II.1.1) :

$$
\frac{d L}{d \lambda}=\varepsilon(\lambda, T]\left[\frac{d L}{d \lambda}\right]_{C N}^{T}+\frac{1}{\pi} R_{C}(\lambda) \frac{d E}{d \lambda}
$$


L'importance relative de chaque contribution dépend essentiellement, pour des objets à température ambiante, de la bande spectrale considérée.

Dans le domaine du visible et du proche infrarouge, le terme prépondérant provient de la réflexion ou diffusion de la lumière ambiante, alors qu'en bande III $(8 / 12 \mu \mathrm{m})$, c'est l'émission propre qui l'emporte. Dans la bande $3 / 5 \mu \mathrm{m}$, les ordres de grandeur des 2 termes sont assez semblables.

Il faut noter aussi que souvent, l'approximation du comportement de surfaces diffusantes par la loi de Lambert peut être abusive et que la connaissance de l'indicatrice de diffusion de la surface ou du milieu peut s'avérer nécessaire, (par exemple pour des surfaces aquatiques, métalliques, ou des surfaces rugueuses observées sous des angles d'incidence importants).

Dans ces circonstances, la géométrie définissant les directions d'illumination et d'observation de chaque surface est importante. II ne suffit plus alors de caractériser une surface diffusante par son facteur de réflexion diffuse $R d(<1)$, mais il faut introduire un paramètre spécifique : le facteur de réflexion bidirectionnelle ou facteur de luminance de la surface dans la direction d'observation $f\left(\lambda, \theta, \theta^{\prime}, \phi, \phi^{\prime}\right)$. Ce paramètre est le rapport entre la luminance de la surface dans la direction d'observation $\left(\theta^{\prime}, \phi^{\prime}\right)$, pour un éclairement provenant de la direction d'illumination $(\theta, \phi)$, et la luminance du diffuseur parfait placé dans les mêmes conditions.

Le facteur de réflexion bidirectionnelle n'est pas normé à 1 comme le facteur de réflexion diffuse et permet de prendre en compte les phénomènes de réflexion quasi spéculaire, de rétroréflexion dûs à la géométrie du diffuseur.

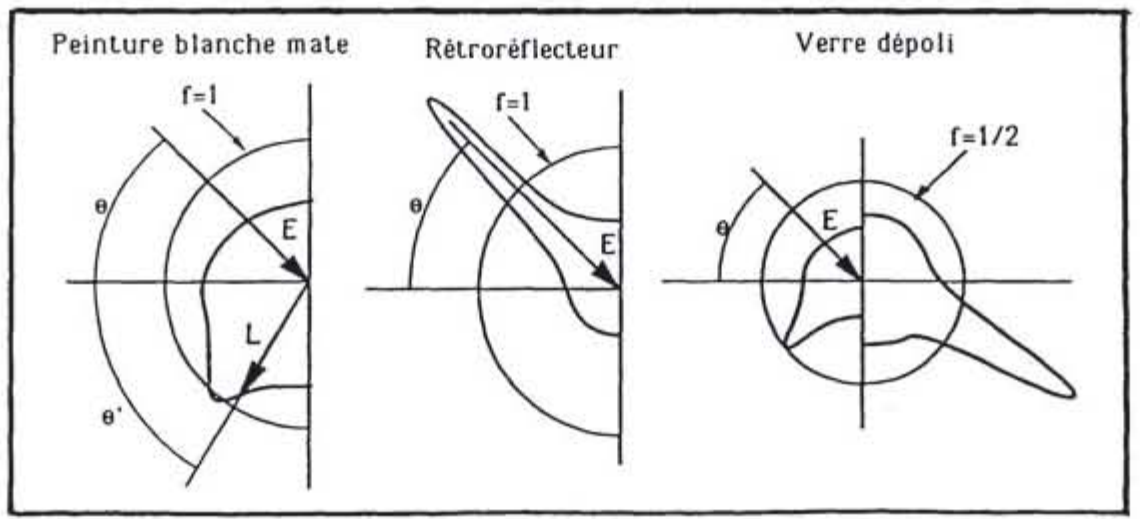

Fig II-6 : Facteur de réflexion bidirectionrıelle

\section{II.2. LE RAYONNEMENT PAR LUMINESCENCE}

\section{II.2.1. GENERALITES}

II a été vu précédemment que toute élévation de température d'un matériau accroît son rayonnement thermique global (loi de Stefan) et en décale le spectre vers les courtes longueurs d'onde (loi du déplacement de Wien).

Si on parvient, sans changement notable de température, mais au moyen d'une excitation sélective en énergie, à hisser préférentiellement les atomes du matériau dans certains des niveaux qui leur sont assignés par la Mécanique Quantique, leur retour au niveau fondamental peut s'accompagner (dans le cas des retours dits "radiatifs") d'une émission de photons, dite émission par luminescence. 
La densité de population des divers états atomiques du milieu n'est plus alors dictée par la statistique de Boltzmann, qui prévaut à l'équilibre thermique, et où la population d'un niveau $\mathrm{N}_{\mathrm{i}}$, d'énergie $E_{i}$ est donnée par: $\quad N_{i}=N_{0} \exp \left(-E_{i} / k T\right)$

L'énergie des photons émis par luminescence est égale à la différence d'énergie entre les niveaux occupés par l'atome avant et après émission :

$$
h v=E_{2}-E_{1}
$$

Le rayonnement par luminescence peut être créé par différents moyens d'excitation des atomes et molécules, parmi lesquels on peut citer:

- l'électroluminescence, excitation électrique par bombardement et collision de ces atomes par des électrons ou ions extérieurs, par injection de charges dans le matériau lui-même (passage de courant électrique)

- la fluorescence, où le changement d'état des atomes s'effectue par l'absorption d'un faisceau optique adapté en longueur d'onde aux niveaux énergétiques du milieu.

- la chimiluminescence, triboluminescence,...

La luminescence s'obtient à partir de milieux en phases gazeuse, liquide ou solide. Les premières sources luminescentes ont été réalisées en milieux gazeux, par l'application d'une différence de potentiel entre 2 électrodes séparées par un gaz ; suivant la densité de courant obtenue, il se produit en général 2 types de rayonnement :

- le premier régime, dit "à décharge" correspond à l'ionisation du gaz par collision de ses atomes ou molécules avec les ions accélérés par le champ électrique. Leur retour au niveau fondamental provoque un rayonnement dont les caractéristiques spectrales dépendent de la composition, pression, température du gaz.

- le deuxième régime, dit "à arc", correspond aux fortes densités de courant, pour lesquelles les électrodes, bombardées par les charges négatives (cas de lanode) et positives (cas de la cathode) s'échangent fortement et où la cathode peut alors émettre des électrons (émission thermoélectronique).

Ces lampes se caractérisent par un spectre d'émission centré sur le visible et le proche infrarouge et leur rendement lumineux ou visuel (voir définition au paragraphe III.1) est particulièrement intéressant. Pour cela, ces lampes sont principalement utilisées dans des applications telles que l'éclairage public (lampes à vapeurs de mercure/sodium ...), le laboratoire (lampes dites "spectrales" à moyenne ou basse pressions pour l'interterométrie et la spectrométrie), le cinéma (lampes à arc au Xénon pour la simulation d'éclairement solaire). Après une lonjue absence, due à lutilisation du tube à rayons cathodiques, elles présentent un intérét certain en visualisation. 


\section{II.2.2. LUMINESCENCE DANS LES SOLIDES}

Remarques:

Comme pour les gaz, l'émission par luminescence dans un solide provient du passage des atomes d'un état excité à un autre de niveau énergétique plus faible. Cependant, il apparaît 2 différences essentielles entre les luminescences dans les milieux gazeux et solides:

- D'une part, dans un solide, les états permis ne sont plus constitués de niveaux discrets, mais de domaines ("bandes" de conduction et de valence) de largeurs énergétiques telles que, en général, l'énergie des photons émis n'est plus aussi étroitement définie que dans les cas des gaz.

- La seconde différence notable entre milieux solides et gazeux porte sur l'efficacité en luminescence. beaucoup plus faible dans un solide que dans un gaz : cette inefficacité en luminescence des solides provient de leur densité d'atomes (nombre par unité de volume) qui est beaucoup plus importante que dans un gaz et qui entraine une forte probabilité d'absorption du rayonnement d'un atome par les atomes voisins (phénomène d'autoabsorption)

- L'adjonction en faible densité "d'impuretés" (ou atomes différents de ceux de la matrice) dans la matrice d'un solide donné, ou "dopage" permet d'améliorer considérablement cette efficacité : lintroduction d'éléments étrangers dans le solide crée des états électroniques dont les niveaux énergétiques se situent dans la "bande interdite" du matériau hôte. Si le retour de l'électron d'un état excité à l'état initial s'effectue par transition sur ces niveaux intermédiaires, l'énergie des photons émis lors de ce retour est inférieure au "gap" énergétique de l'hôte (différence d'énergie entre les bandes de valence et de conduction) et cette désadaptation énergétique rend très faible la probabilité d'absorption de ces photons par les atomes (nombreux) du matériau hôte. De plus, si la densité des atomes d'impureté est faible, la probabilité d'autoabsorption du rayonnement de luminescence de l'impureté par ces propres atomes demeure infime, comme dans le cas d'un gaz (milieu très dilué). On donne ci-après quelques exemples typiques de sources solides à luminescence.

- les lampos à fluorescence

- les diodes électroluminescentes (DEL)

- les tubes à rayons cathodiques.

\section{Lampes à fluorescence}

La fluorescence est l'émission d'un rayonnement par un matériau dont les atomes sont excités par l'absorption d'un autre rayonnement optique. D'après les remarques ci-dessus, les photons du faisceau optique d'excitation sont plus énergétiques que ceux recueillis par fluorescence :

$$
\begin{gathered}
\lambda \text { excitation } \\
\text { (absorption) }
\end{gathered} \quad \underset{\text { émission }}{\lambda \text { (fluorescence) }}
$$

Dans la plupart des cas, les électrons retoument à leur état fondamental en libérant un photon de fluorescence très peu de temps $\left(<10^{-5} \mathrm{~s}\right)$ après leur excitation. II existe cependant des impuretés appelées "pièges" qui par exemple ne restituent leurs électrons qu'au niveau d'énergie supérieure de l'hôte (bande de conduction) sous l'effet de l'agitation thermique et retardent ainsi leur retour à l'état fondamental. En présence de tels pièges, l'émission par fluorescence se trouve très fortement ralentie : on parle alors de "phosphorescence". En réponse à une excitation lumineuse extrèmement brève, le flux émis par phosphorescence décroît exponentiellement en fonction du temps, avec une constante de temps appelée "durée de rémanence". 
Une des illustrations les plus répandues de la fluorescence est la lampe à fluorescence utilisée en éclairage. Ce type de lampe est constitué d'un tube de verre cylindrique (longueur $\sim 1 \mathrm{~m}$; diamètre $\sim 40$ $\mathrm{mm}$ ) contenant du mercure (ainsi que de l'argon et du néon) et deux électrodes à filament de tungstène aux extrémités. La paroi interne du tube est recouverte d'une couche de poudre fluorescente. A la mise sous tension, l'amorçage de l'arc au mercure entraîne une émission dans l'ultra-violet $(\lambda=254 \mathrm{~nm}$ ) qui excite la couche de poudre dont la fluorescence correspond à un ensemble de raies d'émission dans le domaine visible. Le verre du tube, opaque dans l'ultraviolet (pour $\lambda<330 \mathrm{~nm}$ ) assure le blocage du rayonnement émis en direct dans I'UV par la vapeur de mercure.

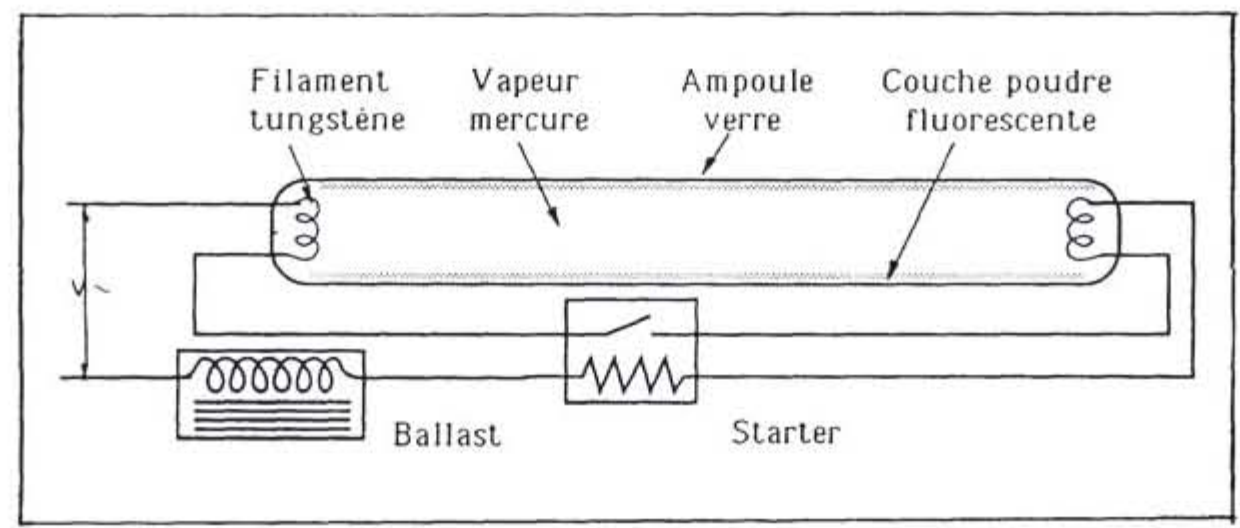

Fig II-7 : Lampe à fluorescence

Diodes électroluminescentes (DEL)

Les diodes électroluminescentes (Light Emitting Diodes ou LED en anglais) sont constituées par des jonctions PN dans des composés semiconducteurs.

On rappelle que, dans un semiconducteur, la substitution en faible proportion d'un élément par un autre de valence plus élevée (par exemple d'un élément pentavalent par un hexavalent) augmente la densité en électrons libres, et assure la conduction électrique du matériau essentiellement par des charges négatives : le matériau est alors dit de type $\mathrm{N}$.

Inversement, le remplacement en faibles quantités d'atomes trivalents par des atomes divalents y augmente la densité des manques d'électrons (trons) qui assurent le transport du courant de conduction : on a affaire dans ce cas à des matériaux de type $P$.

A l'équilibre, il s'établit entre les régions $\mathrm{N}$ et $\mathrm{P}$ d'une jonction PN (assemblage ou contact de ces 2 types de régions dans un même monocristal), une différence de potentiel électrique qui s'oppose à la diffusion des nombreux électrons libres de la région $N$ vers la région $P$, et vice versa pour les trons.

Si la diode est polarisée "en direct" (c'est-à-dire qu'une tension positive est appliquée à la région P par rapport à la région $N$ ), cette barrière de potentiel se trouve réduite : des tlectrons pénètrent de la région $N$ vers la région $\mathrm{P}$ et des trons de la région $\mathrm{P}$ vers la région $\mathrm{N}$. La zone de jonction, "désertée" dans le cas où la diode n'est pas polarisée, devient alors le siège de recombinaison entre électrons et trons qui, si elles sont radiatives, émettent des photons dont l'énergie (inférieure à celle du gap séparant les bandes de valence et de conduction du semiconducteur) dépend de la position du niveau énergétique de l'impureté à l'intérieur de la bande interdite. 
Les DEL les plus courantes, à base de mélanges entre Gallium, Arsenic, Phosphore et Antimoine, émettent dans le visible et le proche IR et s'utilisent essentiellement en visualisation.

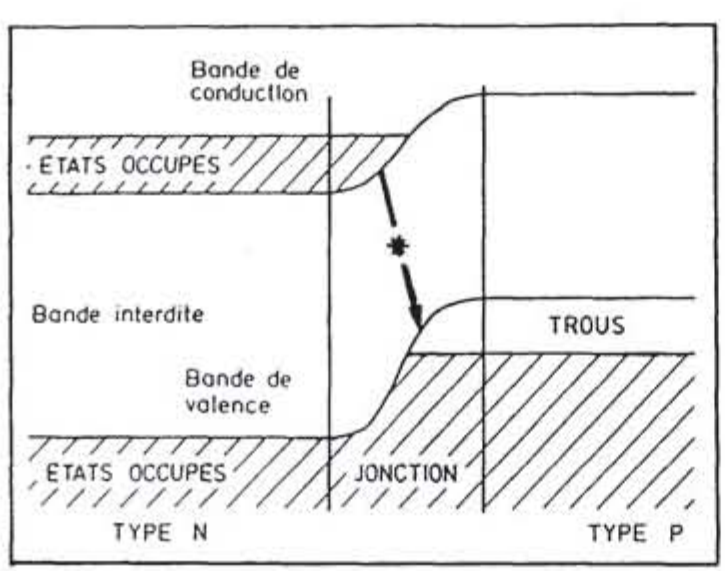

Fig $11-8$ : Jonction PN

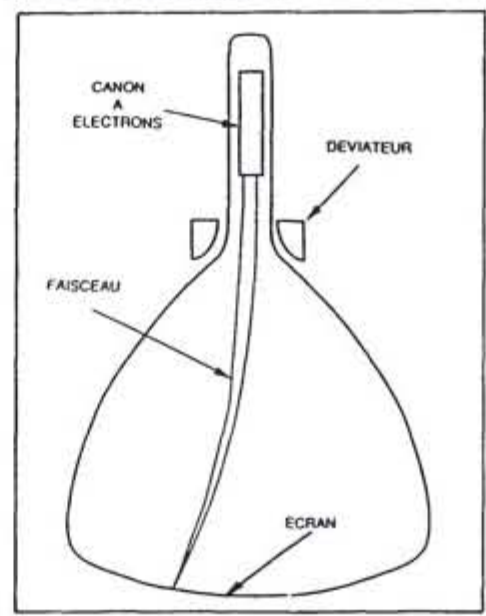

Fig II-9 : Tube à rayons cathodiques

\section{Cathodoluminescence}

L'excitation de matériaux luminescents par le bombardement d'électrons accélérés dans le vide, est très utilisée en visualisation (oscilloscopes, moniteurs de télévision, intensificateurs d'images).

Les électrons émis par une cathode thermoélectronique (ou Wehnelt) sont successivement modulés en débit, accélérés, déviés suivant une loi de balayage appropriée et focalisés sur une poudre luminescente déposée sur la face interne de la fenêtre du tube à vide, au travers d'une anode collectrice très mince (en aluminium). L'optimum de rendement pour ces collisions électroniques se situe à des valeurs en différences de potentiel comprises entre 10 et $20 \mathrm{kV}$ (Fig II- 9).

De nombreuses poudres cathodolumilescentes (ou "phosphores") ont été mises en oeuvre, le choix en vue d'une application particulière se faisant en fonction de paramètres tels que rendement (énergétique. lumineux), propriétés colorimétriques (courbe d'émission spectrale), temps de rémanence (problèmes de "flicker" ou papillotement d'image).

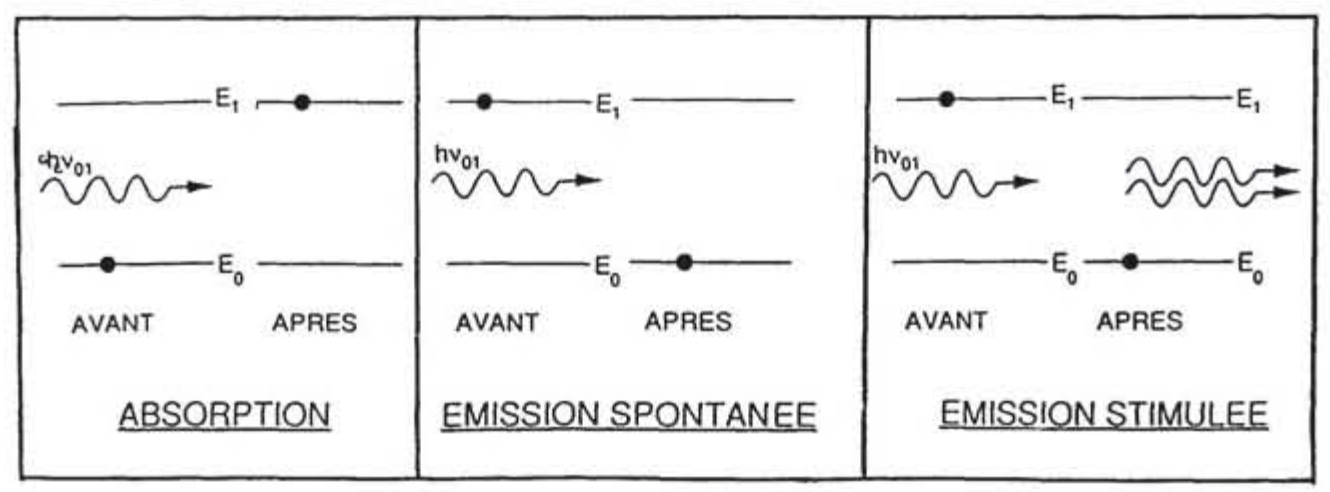

Fig II-10 : Processus d'interaction lumière-matière 


\section{II.3. SOURCES LASER}

Dans l'ensemble des sources optiques, les lasers constituent un groupe à part, non pas parce que le flux qu'ils émettent est en moyenne supérieur à celui des sources conventionnelles (c'est plutôt linverse) mais parce qu'il peut être délivré sous des formes (spatiale, spectrale et temporelle) difficilement accessibles par les moyens traditionnels, ce qui rend les lasers attractifs, voire indispensables dans un nombre grandissant d'applications.

IIs se diversifient de plus en plus dans leur constitution et leurs utilisations, allant de sources miniatures (diodes laser) pour télécomınunications aux projets volumineux pour la fusion nucléaire ou l'initiative de Défense Stratégique.

\section{II.3.1. RAPPELS DE BASE}

Une source laser est essentiellement un oscillateur optique, c'est-à-dire un amplificateur bouclé sur lui-même, qui comprend les éléments de base suivants :

- Un milieu optique dont les atomes et/ou molécules sont excités sélectivement et qui joue le rôle d'amplificateur, par "émission stimulée", de son propre rayonnement, que lui ré-injecte partiellement :

- un résonateur ou cavité optique de type Fabry-Pérot, englobant le milieu considéré ci-dessus.

On rappelle ici les 3 processus d'interaction de la lumière avec la matière (Fig II-10) et qui interviennent dans le fonctionnement d'un laser : le premier ("émission spontanée") consiste en l'émission d'un photon par un atome lors du retour radiatif d'un électron depuis un niveau excité à un niveau d'énergie plus faible. Le second processus ("absorption") résulte en l'excitation d'un électron initialement à l'état fondamental et la disparition d'un photon lors de la collision du premier par le second. Le troisième processus ("émission stimulée") consiste en l'interaction d'un photon avec un atome (énergétiquement adapté au photon) préalablement excité et résulte en l'émission par cet atome de 2 photons "identiques" (en énergie, direction de propagation, état de polarisation).

Dans une source thermique, ou à luminescence, l'émission spontanée constitue l'essentiel du rayonnement utile de la source. Dans un laser, le rayonnement par luminescence du matériau le long de l'axe de la cavité sert "d'amorce" à un flux dont une fraction est renvoyée par les miroirs de cette cavité sur le milieu lui-mêrne, où elle est amplifiée (par émission stimulée) lors de ses passages successifs.

Ce phénomène d'oscillation ne se manifeste que dans la direction perpendiculaire aux miroirs et pour certaines fréquences sélectivement entretenues par la géométrie de la cavité. Dans le cas, simple, d'une cavité Fabry-Pérot à miroirs plans séparés d'une distance $\mathrm{L}$ par un milieu d'indice de réfraction $\mathrm{n}$, les fréquences des modes successifs de la cavité (modes tels que l'onde se retrouve en phase avec elle-même après un aller/retour dans la cavité) sont telles que :

$$
v_{N}=N \frac{c}{2 n L}
$$

En fonction du rapport entre la largeur spectrale de la transition laser (entre les niveaux excité et fondamentale), exprimée en $\mathrm{Hz}$ et la distance intermodale $(\mathrm{c} / 2 \mathrm{~nL})$ imposée par la distance entre ses miroirs, l'énergie dans la cavité se répartit sur un certain nombre de modes. L'un des 2 miroirs de la cavité ayant un facteur de transmission non nul, une partie de l'énergie optique de la cavité se propage à l'extérieur où elle constitue le rayonnement utile du laser, qui sera dit monomode ou multimode, suivant le nombre de modes émis. 
Tableau II-2 : Caractéristiques modales de lasers typiques

\begin{tabular}{|l|c|c|c|c|c|}
\hline \multicolumn{1}{|c|}{ Laser } & $\lambda(\mathrm{nm})$ & $\Delta v(\mathrm{MHz})$ & $L(\mathrm{~cm})$ & $\Delta v \mathrm{~L}(\mathrm{MHz})$ & $\begin{array}{c}\text { Nombre de } \\
\text { modes }\end{array}$ \\
\hline Argon & $488,514,5$ & 3500 & 100 & 150 & $\sim 20$ \\
\hline He-Ne & & 1500 & 20 & 750 & $\sim 2$ \\
\hline Rubis $\left(\mathrm{T}=300^{\circ} \mathrm{K}\right)$ & 694,3 & $310^{5}$ & 10 & 1500 & $\sim 200$ \\
\hline Rubis $\left(\mathrm{T}=77^{\circ} \mathrm{K}\right)$ & 694,3 & $10^{4}$ & 10 & 1500 & $\sim 6$ \\
\hline AsGa & 905 & $1,210^{6}$ & 0,2 & 75000 & $\sim 15$ \\
\hline $\mathrm{Nd}:$ verre & 1060 & $610^{6}$ & 10 & 1500 & $\sim 4000$ \\
\hline $\mathrm{Nd}:$ YAG & 1060 & $1,210^{5}$ & 100 & 150 & $\sim 800$ \\
\hline $\mathrm{CO}_{2}$ (basse pression) & 10600 & 60 & 100 & 150 & $\sim 1$ \\
\hline $\mathrm{CO}_{2}$ (TEA) & 10600 & 3500 & 30 & 500 & $\sim 7$ \\
\hline $\mathrm{CO}_{2}$ (guide d'ondes) & 10600 & 500 & 20 & 750 & $\sim 1$ \\
\hline
\end{tabular}

\section{II.3.2. EXEMPLES DE LASER}

L'amplification de lumière par émission stimulée se manifeste aussi bien dans les solides que les liquides ou les gaz, avec les mêmes remarques que pour la luminescence (nécessité de dopage à faibles doses dans les solides), ce qui donne lieu à la réalisation de sources lasers à partir de milieux amplificateurs dans ces 3 phases, l'excitation du milieu étant soit électrique, soit optique ("pompage optique"). En pratique, les lasers les plus répandus actuellement sont les lasers à solides (laser à semi-conducteurs, lasers Yag dopés au Néodyne,...) et les lasers à gaz (lasers Hélium-Néon, $\mathrm{CO}_{2}, \ldots$ )

\section{Lasers à gaz}

En général, le milieu émetteur/amplificateur d'un laser à gaz est un gaz sous basse pression excité par décharge électrique dans un tube scellé. Les extrémités du tube peuvent supporter directement les miroirs de la cavité ou être scellées par des fenêtres orientées à l'angle de Brewster, les miroirs étant alors indépendants du tube. Dans ce dernier cas, le rayonnement uu laser est polarisé linéairement dans le plan d'incidence des lames de Brewster. Le schéma de la Figure II-11 représente un tel laser à gaz Hélium-Néon. Les tensions appliquées aux électrodes sont typiquement de l'ordre de 10 à $20 \mathrm{kV}$.

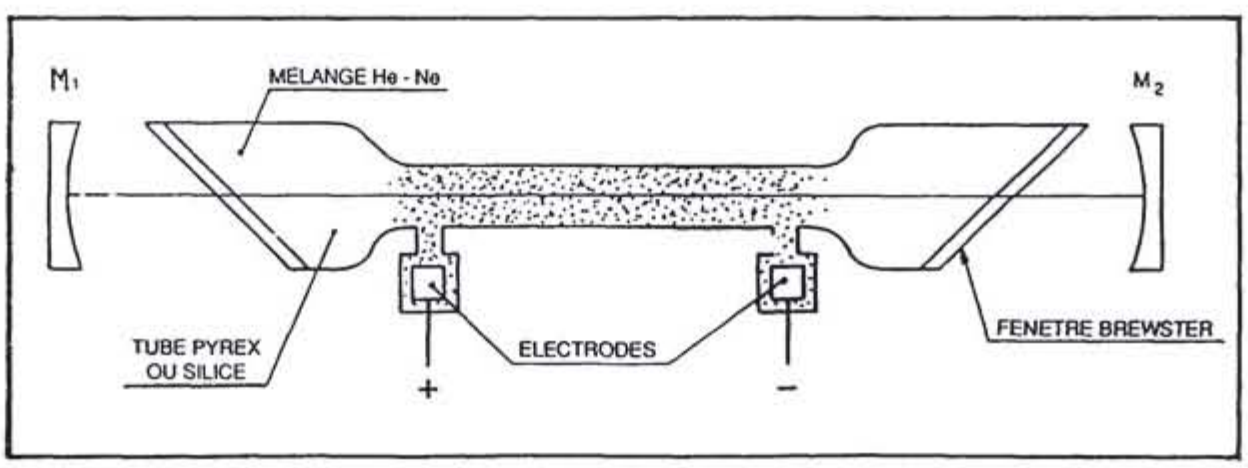

Fig II-11: Schéma d'un laser HeNe 
Les lasers à gaz tels que lasers $\mathrm{HeNe}$ ou $\mathrm{CO}_{2}$ contiennent en fait des mélanges gazeux : dans le cas du laser $\mathrm{He}-\mathrm{Ne}$, l'excitation électrique se fait sur les atomes de Néon, avec transfert d'énergie par collision atomique sur l'hélium. Dans les lasers $\mathrm{CO}_{2}$ (mélange $\mathrm{CO}_{2}+\mathrm{N}+\mathrm{He}$ ), l'excitation initiale concerne l'azote, avec transfert d'énergie par collision aux molécules de $\mathrm{CO}_{2}$.

Jusqu'à présent, les lasers à gaz sont ceux qui permettent d'obtenir les surfaces d'onde de qualité optique optimale (émission monomode, stabilité en fréquence, divergence limitée par la diffraction), ce qui les rend aptes aux applications scientifiques les plus exigentes (métrologie, interférométrie, holographie. détection hétérodyne).

De plus, certains lasers à gaz, tels que le laser $\mathrm{CO}_{2}$, se prêtent aussi à des applications industrielles (découpe, soudure), médicales (chirurgie) ou militaires (télémétrie, guidage de missile) pour lesquelles le flux émis doit être assez important.

Lasers solldes

Dans les lasers à solides, tels que le laser à rubis $\left(\mathrm{AL}_{2} \mathrm{O}_{3}\right.$ dopé au $\left.\mathrm{Cr}_{2} \mathrm{O}_{3}\right)$ ou le laser $\mathrm{Nd}-\mathrm{Yag}$ (Grenat d'Ythrium Aluminium dopé par $\mathrm{Nd}_{2} \mathrm{O}_{3}$ ), la cavité est constituée par le cristal lui-même dont les 2 faces extrêmes constituent les miroirs après polissage et traitement par couches minces.

Ces lasers constituent des sources extre̊mement robustes, en particulier les lasers Nd-Yag, qui s'utilisent aussi bien dans les applications scientifiques (projets de fusion par laser) qu'industrielles (découpe) ou militaires (télémétrie, désignation laser de cible).

Les lasers à solides les plus nombreux sont les lasers à semi-conducteurs ou diodes laser, qui sont des jonctions PN, comme les DEL. L'émission du flux s'y produit non pas perpendiculairement à la jonction comme dans le cas d'une DEL, mais parallèlement.

Ces dispositifs forment des émetteurs optiques très compacts, montés par l'une de leurs électrodes sur une embase métallique utilisée comme absorbeur thermique. A cause de la différence importante entre les 2 dimensions de l'émetteur (la jonction peut mesurer $1 \mu \mathrm{m}$ en épaisseur par quelques dizaines de $\mu \mathrm{m}$ en longueur) le faisceau émis possède lui-même une dissymétrie dans les 2 plans parallèle et perpendiculaire à la jonction. De nombreux travaux sont en cours sur ce type de sources, en particulier dans les domaines des télécommunications par fibres optiques (diodes laser de grande bande passante à $\lambda=1,5 \mu \mathrm{m}$ ) ou des diodes laser de puissance, pour le pompage optique de lasers à solides.

\section{II.3.3. CONSIDERATIONS SUR LA LUMINANCE DES LASERS}

L'un des avantages du laser est sa directivité angulaire qui peut conduire à l'obtention de faisceaux limités par la diffraction, à luminances très élévées.

Par exemple, l'indicatrice en intensité d'un laser monomode (TEMoo) a un profil gaussien centré sur l'axe d'émission:

$$
k(\theta)=l_{0} e^{-2 \theta / \theta_{0}}
$$

où $\theta 0$, le demi-angle au sommet du cône définissant la divergence du faisceau à grande distance est donné par:

$$
\theta_{0}=\lambda \pi \omega_{0}
$$

La dimension transversale du faisceau dans un plan perpendiculaire à l'axe de propagation situé à une abscisse est donnée traditionnellement par la valeur du rayon à $1 / \mathrm{e}^{2}, w(z)$, défini comme la distance à l'axe pour laquelle l'éclairement laser est égal à $1 / e^{2}$ fois sa valeur sur l'axe (voir Figure II-12).

On montre que :

$$
\omega^{2}(z)=\omega_{0}^{2}\left[1+\left(\frac{\lambda_{z}}{\pi \omega_{0}^{2}}\right)^{2}\right]
$$


Expérimentalement, on peut vérifier de diverses façons si le laser utilisé fonctionne dans ce mode fondamental, par exemple en plaçant un diaphragme de diamètre variable au foyer d'une lentille de longue focale, éclairée par le laser. La courbe obtenue donnant l'énergie laser "encerclée" par le diaphragme en fonction de son diamètre permet de vérifier le caractère gaussien de lintensité et d'en déduire la divergence à $1 / e^{2}$ (pour laquelle l'énergie encerclée est $87 \%=1-1 / e^{2}$ de l'énergie totale, mesurée sans diaphragmation. II existe aussi sur le marché des analyseurs de faisceau, constitués de barrettes linéaires de détecteurs de petite taille, indépendants les uns de autres. La mesure de la réponse relative des détecteurs lorsque la barrette est placée dans le faisceau permot de vérifier la loi d'éclairement laser.

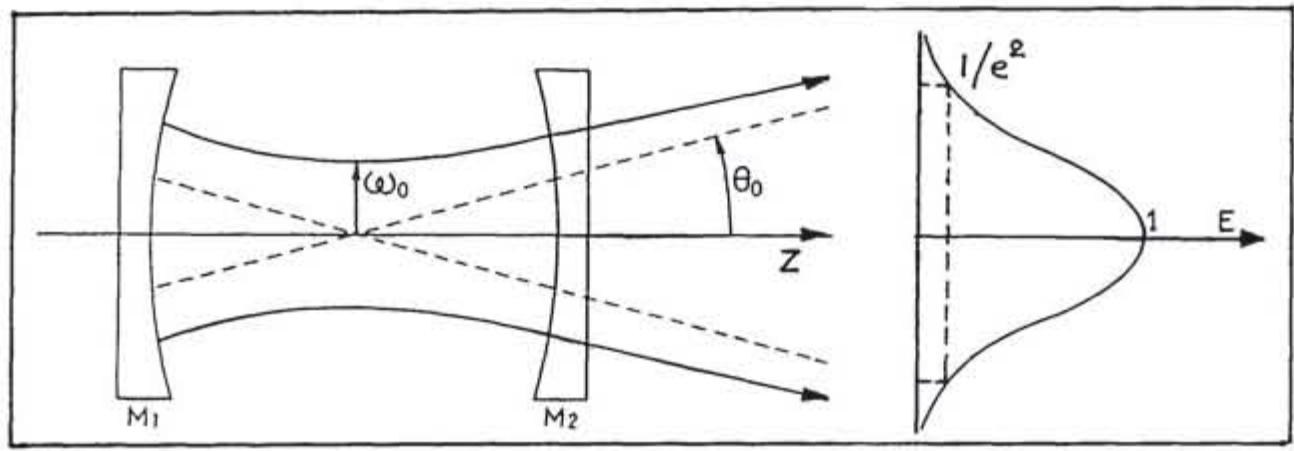

Fig $11-12$ : Laser monomode TEMoo

On peut évaluer approximativement la luminance moyenne $\mathrm{Lm}$ du rayonnement d'un laser monomode TEMoo à l'intérieur de son lobe d'émission en assimilant l'étendue géométrique du faisceau émis à celle d'une source dont la surface est celle d'une source dont la surface est celle du "beam waist" du laser et dont la luminance est uniforme à lintérieur du cône de demi-divergence $\Theta_{0}$.

Cette étendue géométrique, qui est celle de tout faisceau limité par la diffraction, est égale à

$$
G=S \Omega=\left[\pi \omega_{0}^{2}\right] \times \pi\left(\lambda / \pi \omega_{0}\right)^{2}=\lambda^{2}
$$

La luminance sur l'axe du laser est donc:

$$
L L=F L / G=F L / \lambda^{2}
$$

si $F L$ est le flux émis par ce laser.

Exercice :

Comparer la luminance sur l'axe d'un laser HeNe monomode TEMoo (flux émis $=1 \mathrm{~mW}$ ) avec celle du soleil.

$$
L_{\mathrm{HeNe}} \sim 10^{-3} /\left(0,6^{2} \times 10^{-12}\right)=310^{9} \mathrm{Wm}^{-2} \mathrm{si}^{-1}
$$

Cette luminance de laser modeste est plus de 100 fois supérieure à la luminance globale du soleil dans tout le spectre (rappel : $L S=K_{3} T S^{4}=2,210^{7} \mathrm{~W} \mathrm{~m}^{-2} \mathrm{sr}^{-1}$ ). 


\section{II.3.4. RENDEMENT ENERGETIQUE DES LASERS}

Le rendement énergétique d'un laser, qui s'exprime par le rapport entre le flux optique émis et la puissance électrique consommée, dépend de nombreux paramètres, tels que l'efficacité d'excitation des atomes du milieu amplificateur, leur durée de vie dans le niveau excité, le rapport entre les longueurs d'onde d'excitation et d'émission (dans le cas de pompage optique), l'adaptation de la cavité au milieu amplificateur, les facteurs de réflexion des miroirs, les pertes par diffraction dans la cavité, etc...

II en résulte que les rendements des lasers diffèrent grandement d'un laser à un autre : on peut citer comme résultats typiques : $0,5 \%$ pour un laser $\mathrm{Nd}$ Yag (à pompage par flash), $10 \%$ pour un laser $\mathrm{CO}_{2}$. 20 à $30 \%$ pour une diode laser.

Pour un type de laser donné, ces caractéristiques peuvent s'améliorer considérablement en fonction des résultats de recherche, en particulier sur l'optimisation des méthodes d'excitation du milieu amplificateur. Les progrès obtenus actuellement dans le domaine du laser Nd Yag en sont une illustration :

Des études récentes ont démontré lintérêt du pompage de tels lasers par des diodes laser dont la longueur d'onde d'émission peut être adaptée finement au pic d'aborption du Néodyme. Le passage du pompage par arc au pompage par diode peut faire passer le rendement de ces lasers de $0,5 \%$ à $6 \%$.

\section{II.3.5. SECURITE LASER}

Le laser se trouve de plus en plus utilisé en dehors du laboratoire, dans des domaines tels que la construction et les chantiers (alignement), limprimerie, la chirurgie, les supermarchés (lecture de codes), le spectacle ("laser shows"), l'armement (télémétrie laser, Lidar), etc... La luminance très élevée du laser en fait une source de lumière potentiellement dangereuse. Le présent paragraphe a pour but de présenter une évaluation simple de ces dangers et de rappeler les principales règles de sécurité laser.

\section{Rappel : Effets thermiques des radiations électromagnétlques}

Une radiation électromagnétique incidente sur un matériau qugiconque y génère des effets thermiques (variation de température) dépendant essentiellement de :

- l'éclairement énergétique $\left(\mathrm{Wm}^{-2}\right)$ de la surface du matériau dû à la radiation lorsque cette demière émet continûment. Si la source est impulsionnelle, on considère soit la lumination énergétique $\left(\mathrm{Jm}^{-2}\right)$ par impulsion, soit léclairement énergétique moyen.

- le facteur d'absorption du matériau à la longueur d'onde de la radiation incidente. Ce facteur détermine lẹ pourcentage d'énergie incidente transformée en chaleur dans le matériau irradié.

Les dangers potentiels d'un faisceau laser concernent principalement la peau et les yeux de l'utilisateur. L'oeil est de beaucoup le plus vulnérable, en particulier dans le visible et le proche infrarouge $(0,4 \mu \mathrm{m}<\lambda$ $<1,4 \mu \mathrm{m}$ ), domaine où la transparence des milieux oculaires permet à un faisceau quasi parallèle de se focaliser sur une très petite surface de la rétine grâce à la comée et au cristallin (voir Figure II-13).

En effet, dans le cas d'un faisceau incident quasi parallèle (vision directe d'un faisceau laser), les rayons collectés par la pupille se concentrent sur une surface d'une centaine de microns carrés au niveau de la rétine : cette focalisation y provoque donc un éclairement local environ $10^{5}$ fois plus fort que sur la pupille ou sur la peau et fait de cet organe le point le plus vulnérable au faisceau laser.

Dans les autres domaines spectraux (en particulier $\lambda>1,4 \mu$ ), tous les milieux oculaires sont opaques et absorbent le rayonnement laser incident bien avant qu'il n'atteigne la rétine. 
La peau présente aussi un facteur d'absorption très important dans l'infrarouge et les normes de sécurité laser pour la peau et pour l'oeil sont alors beaucoup plus proches (lasers dits "à sécurité oculaire" ou "Eye safe").

\section{Classification générale des lasers}

Les normes de sécurité classent généralement les lasers en 4 catégories. Par exemple, la norme américaine ANSI Z136 (American National Standard Institute) pour la sécurité laser donne la classification suivante :

- La classe l est constituée par les lasers les moins puissants, considérés comme non dangereux dans tous les cas. En pratique, elle se limite aux lasers à Arseniure de Gallium.

- Dans la classe II, on trouve les lasers émettant dans le visible et qui seraient dangereux pour l'oeil d'un observateur qui fixerait directement le faisceau pendant une période assez longue (quelques secondes).

- Les lasers ou systèmes laser de la classe III peuvent endommager l'oeil de l'observateur en vision directe même dans le cas d'une exposition très brève (par exemple inférieure à $0,25 \mathrm{~s}$, temps de réponse du réflexe de protection oculaire). Cependant, ces lasers ne sont dangereux ni pour la peau en direct, ni pour l'oeil après une réflexion diffuse.

- La classe IV représente les lasers à haut risque. Ils peuvent être dangereux pour l'oeil même après réflexion diffuse et brôler sévèrement la peau par exposition directe.

Aspect quantitatif

Les normes de sécurité laser résultent d'expérimentations sur le comportement de divers tissus représentatifs de la peau ou de l'oeil face à un éclairement laser. Elles donnent des tableaux de valeurs maximales permissibles d'éclairement ou d'exposition laser sur la peau ou la pupillle. Sans entrer dans le détail des chiffres, on constate que ces niveaux dépendent des paramètres suivants :

- Longueur d'onde d'émission du laser

- Caractère continu ou impulsionnel de l'émission

- durée d'impulsion et fréquence de répétition pour les lasers impulsionnels

- Étendue géométrique du faisceau incident (vision directe du faisceau ou après réflexion sur un diffuseur)

- Diamètre de la pupille de l'observateur (les normes considèrent des diamètres de $1 \mathrm{~mm}$ ou $7 \mathrm{~mm}$ )

- La couleur de la peau

La Figure II-14 donne un tel tableau de valeurs d'éclairements maximaux permissibles dans le cas de la vision directe (faisceau collimaté).

Pour évaluer le niveau de sécurité correspondant à chaque cas concret, on peut consulter les références suivantes:

- "Safety with Lasers and other Optical Sources", A Comprehensive hanobook, David SLINEY and Myron WOLBARSHT - Plenum Press, 1980

- Norme ANSIZ 136

- Norme NATO "STANAG 3606 LAS"

- Norme BRH (Bureau of Radiological Health) 


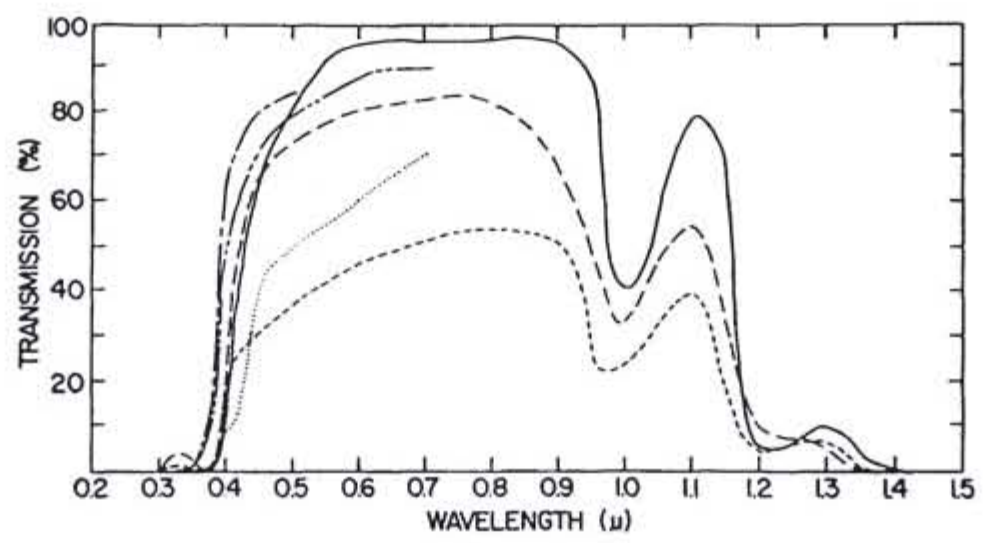

Fig II-13: Transmission spectrale des milieux oculaires

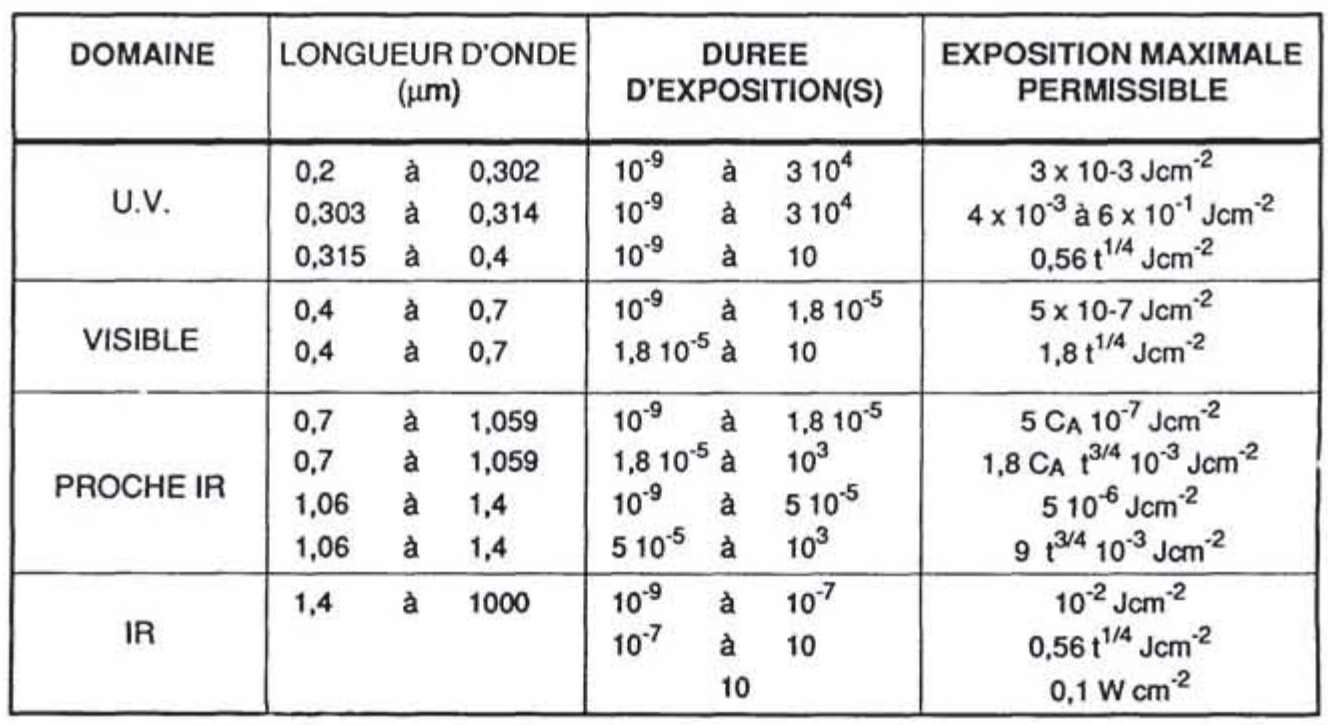

Fig II-14 : Exemples de niveaux d'exposition maximale permissible pour une vision directe d'un falsceau laser 


\section{CHAPITRE 3}

\section{RECEPTION DU RAYONNEMENT}

Ce chapitre traite des détecteurs qui constituent l'un des éléments de base dans le bon fonctionnement d'un système optique : en absorbant les photons captés par les composants optiques du système, le détecteur convertit leur énergie en un signal mesurable (signal électrique) ou interprétable par l'homme (imagerie,...).

Le paragraphe III.1 présente tout d'abord les caractéristiques du détecteur le plus universellement utilisé, l'oeil humain, en tant que base du système d'unités lumineuses (ou visuelles). Les 2 paragraphes suivants définissent les paramètres de base utilisés pour la caractérisation des détecteurs et en donnent les expressions correspondantes dans le mode d'utilisation de détecteurs actuellement le plus répandu en optique, c'est-à-dire la détection directe ou incohérente (paramètres de signal au paragraphe III.2 et de bruit au paragraphe III.3). Le paragraphe III.4 traite de la détection hétérodyne ou cohérente du signal optique, qui se répand de plus en plus dans les applications laser. Enfin, le dernier paragraphe de ce chapitre (paragraphe III.5) décrit succintement les caractéristiques de détecteurs typiques.

\section{III.1. L'OEIL ET LE SYSTEME D'UNITES PHOTOMETRIQUES VISUELLES}

Objet de la photométrie visuelle

L'observation d'une scène par un être humain, en direct ou grâce à un système optique, se traduit par un ensemble de stimulations visuelles interprêtées par le cerveau sous formes de couleurs et de niveaux de lumière, dont l'étude a donné naissance à 2 disciplines, assez indépendantes l'une de l'autre : la colorimétrie et la photométrie visuelle. La première, qui concerne l'aralyse des impressions colorées, n'entre pas dans le cadre de ce cours.

Quant à la photométrie visuelle, son objet est de quantifier en ni reaux la stimulation visuelle d'un observateur par une source optique. Elle cherche donc à traduire en mesures aussi fiables et répétitives que possible, les expressions peu précises mais couramment utilisées pour décrire ces stimulations (on parle de sources lumineuses "intenses", "brillantes", "faibles", etc....).

Cette mission peut paraître ambitieuse dans la mesure où les sensations lumineuses sont assez différentes d'une personne à l'autre et où l'impression visuelle ressentie par un observateur face à une source donnée peut elle-même se modifier de façon importante suivant l'åge, les conditions d'éclairement ambiantes ou antérieures, l'état de fatigue du sujet : il est bien connu qu'un automobiliste trouve "noir" un tunnel sombre dans lequel il pénètre et qu'à la sortie, il est ébloui par le paysage extérieur auquel il s'était adapté avant le passage dans le tunnel.

Pour satisfaire les besoins de l'industrie (en particulier, l'éclairagisme) dans la caractérisation objective des propriétés lumineuses des instruments optiques, la CIE (Commission Internationale de l'Eclairage) a défini un système d'unités basé sur une moyer rie de résultats de mesures obtenus à partir d'un grand nombre d'observateurs et définissant un "oeil standard de l'observateur CIE".

La photométrie visuelle s'appuie d'une part sur une caractérisation en sensibilité spectrale relative de l'oeil et d'autre part sur le raccordement avec les unités énergétiques.

Sensiblilté spectrale relative de l'oell

Par définition du domaine "visible" on dit que l'oeil humain est "sensible" aux rayonnements électromagnétiques de longueurs d'onde comprises entre $0,4 \mu \mathrm{m}$ et $0,8 \mu \mathrm{m}$. 
II faut noter que cette sensibilité n'est pas uniforme à l'intérieur de ce domaine spectral, car, si l'on observe une source lumineuse quasi monochromatique, limpression lumineuse ressentie dépend de la longueur d'onde d'émission de la source, si le flux énergétique de cette dernière est maintenu constant.

On constate expérimentalement que l'impression visuelle croît continûment d'une valeur nulle dans l'ultraviolet à une valeur maximale au centre du spectre visible, pour redécroitre ensuite et devenir nulle dans l'infrarouge proche. Les courbes exprimant l'évolution du rapport entre la valeur de l'impression visuelle ressentie à chaque longueur d'onde (hors la couleur) et sa valeur maximale sont appelées courbes de sensibilité spectrale relative de l'oeil et sont propres à chacun.

L'ensemble des mesures obtenues sur un certain nombre d'expérimentateurs a conduit la CIE à définir 2 courbes standard de sensibilité relative de l'oeil, utilisables selon les conditions d'éclairement ambiant dans lesquelles s'effectue l'observation. La première, $V(\lambda)$, qui correspond a la vision dite de jour ou photopique et à des niveaux de luminance ambiante supérieurs à $10 \mathrm{~cd} / \mathrm{m}^{2}$, met en jeu la vision par les cônes de la rétine. La seconde courbe $V^{\prime}(\lambda)$ définissant la vision par les bâtonnets, correspond aux observations dites "de nuit" ou scotopiques pour lesquelles la luminance ambiante est inférieure à $10^{-4}$ $\mathrm{Cd} / \mathrm{m}^{2}$. Pour les niveaux ambiants intermédiaires (de luminance comprise entre $10^{-4}$ et $10 \mathrm{~cd} / \mathrm{m}^{2}$ ), la vision est dite mésotopique et la courbe correspondante dépend du niveau.

Pour les visions photopique et scotopique, la Figure III-1 représente les courbes de sensibilité spectrale relative $V(\lambda)$ et $V^{\prime}(\lambda)$. On constate que le maximum de réponse de l'oeil en vision de jour se situe à $\lambda=$ $0,555 \mu \mathrm{m}$ et en vision de nuit à $\lambda=0,510 \mu \mathrm{m}$ (le décalage de la courbe vers les courtes longueurs d'onde en vision scotopique se dénomme "l'effet Purkinje"). Les valeurs numériques de ces courbes sont données sur le tableau III-1 (a et b) Tableau III-1 a : Valeurs $V(\lambda), V(\lambda)$

\begin{tabular}{|l|l|l|}
\hline $\begin{array}{c}\lambda \\
\mathrm{nm}\end{array}$ & Photopic $\mathrm{V}(\lambda)$ & Scotopic $\mathrm{V}^{\prime}(\lambda)$ \\
\hline 380 & 0.00004 & 0.00059 \\
390 & 0.00012 & 0.00221 \\
400 & 0.0004 & 0.00929 \\
410 & 0.0012 & 0.03484 \\
420 & 0.0040 & 0.0966 \\
430 & 0.0116 & 0.1998 \\
440 & 0.0230 & 0.3281 \\
450 & 0.0380 & 0.4550 \\
460 & 0.0600 & 0.5672 \\
470 & 0.0910 & 0.6756 \\
480 & 0.1390 & 0.7930 \\
490 & 0.2080 & 0.9043 \\
500 & 0.3230 & 0.9817 \\
510 & 0.5030 & 0.9966 \\
520 & 0.7100 & 0.9352 \\
\hline
\end{tabular}

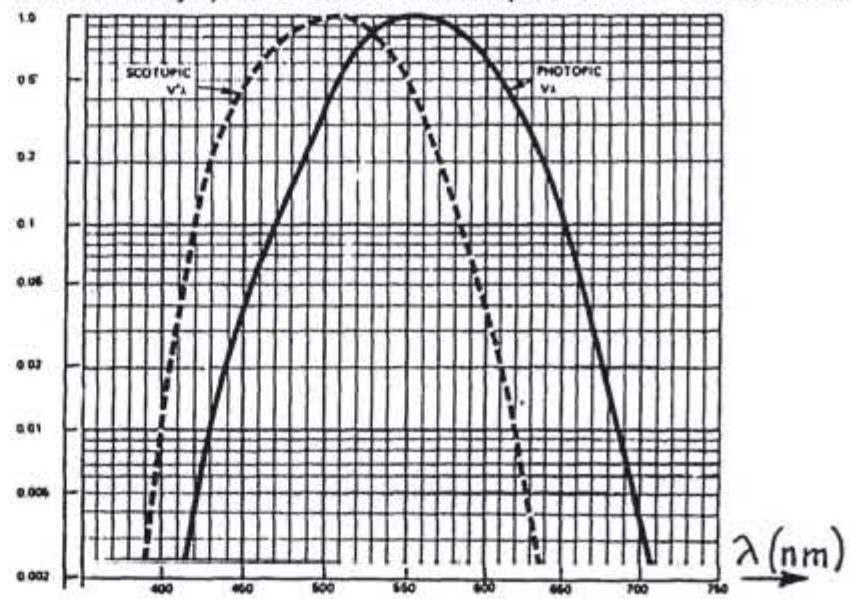

Figure III-1 : Courbes de sensibilité spectrale relative de l'oeil

Raccordement absolu entre systèmes lumineux et énergétique Jusqu'en 1978, les unités du système lumir eux (ou visuel) étaient reliées aux unités énergétiques à partir de la définition de lintensité lumineuse de sources étalons à spectres larges (bougies, Corps Noir). Depuis cette date, le raccordement entre les 2 systèmes est basé sur une correspondance entre unités dans le cas de sources quasi monochromatiques. Deux coefficients de transfert ont été définis, le premier $K_{m}$ pour la vision photopique et le second $K_{m}$ pour la vision scotopique, de telle sorte que :

- en vision photopique, le rapport entre les flux énerg $\in$ tique Fe et visuel Fv d'un rayonnement au maximum de sensibilité de l'oeil $\left(\lambda \mathrm{m}=0,555 \mu \mathrm{m}\right.$ ou $\left.\eta=54010^{12} \mathrm{~Hz}\right)$ soit égal à $\mathrm{K}_{\mathrm{m}}$ : 
- en vision scotopique, pour un rayonnement à $\lambda^{\prime} m=0,510 \mu \mathrm{m}$, ce rapport est :

Pour toute autre longueur d'onde, le rapport entre les flux visuel et énergétique d'un rayonnement quasi monochromatique se trouve atténué vis à vis de ces valeurs optimales à cause de la dégradation en sensibilité spectrale de l'oeil de part et d'autre de $\lambda_{m}$ et $\lambda^{\prime} m$.

Le flux lumineux (en lumens) d'un rayonnement quasi monochromatique de flux énergétique $\mathrm{Fe}$ (en W) à toute longueur d'onde $\lambda$ est donc égal a :

$$
\begin{array}{ll}
F_{V}(\lambda)=K_{m} V(\lambda) F_{\theta}(\lambda) & \text { en lumens dits photopiques (vision de jour) } \\
F_{V}(\lambda)=K^{\prime}{ }_{m} V^{\prime}(\lambda) F_{\theta}(\lambda) & \text { en lumens dits scotopiques (vision de nuit) }
\end{array}
$$

Les valeurs numériques de $\mathrm{K}_{\mathrm{m}}(683 \mathrm{~lm} / \mathrm{W})$ et $\mathrm{K}_{\mathrm{m}}(1703 \mathrm{~lm} \mathrm{~W})$ ont été choisies de façon à raccorder au mieux le système visuel actuel avec son prédécesseur (basé sur la définition de la candela comme intensité lumineuse de $1 / 60 \mathrm{~cm}^{2}$ de Corps Noir à la température de fusion du platine, $2042 \mathrm{~K}$ ).

La Figure III-2 traduit pour chaque longueur d'onde les coefficients de transfert absolu $\mathrm{K}_{\mathrm{m}} \mathrm{V}(\lambda)$ et $\mathrm{K}_{\mathrm{m}}$ $V^{\prime}(\lambda)$ entre unités énergétiques et unités visuelles applicables aux rayonnements quasi monochromatiques.

Tableau III-1 b: Valeurs $V(\lambda) V^{\prime}(\lambda)$

\begin{tabular}{|l|l|l|}
\hline $\begin{array}{c}\lambda \\
\text { nm }\end{array}$ & Photopic $V(\lambda)$ & Scotopic $V^{\prime}(\lambda)$ \\
\hline 530 & 0.8620 & 0.8110 \\
540 & 0.9540 & 0.6497 \\
550 & 0.9950 & 0.4808 \\
560 & 0.9950 & 0.3288 \\
570 & 0.9520 & 0.2076 \\
580 & 0.8700 & 0.1212 \\
590 & 0.7570 & 0.0655 \\
600 & 0.6310 & 0.03325 \\
610 & 0.5030 & 0.01593 \\
620 & 0.3810 & 0.00737 \\
630 & 0.2650 & 0.003335 \\
640 & 0.1750 & 0.001497 \\
650 & 0.1070 & 0.000677 \\
660 & 0.0610 & 0.0003129 \\
670 & 0.0320 & 0.0001480 \\
680 & 0.0170 & 0.0000716 \\
690 & 0.0082 & 0.00003533 \\
700 & 0.0041 & 0.00001780 \\
710 & 0.0021 & 0.00000914 \\
\hline
\end{tabular}

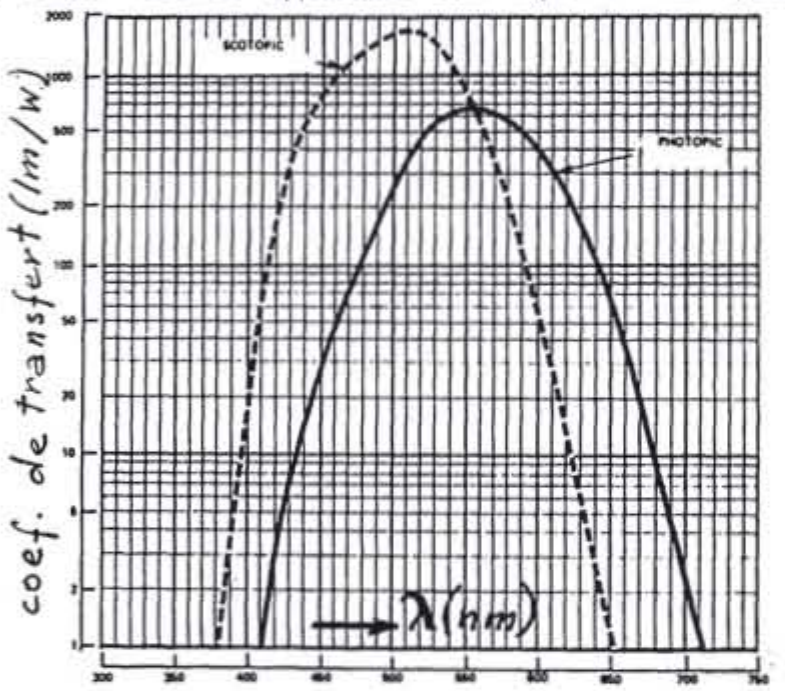

Flg III-2 : Coefficients de transfert absolu entre unltes visuelles et énergétiques (rayonnement monochromatique)

Dans le cas de lumières polychromatiques, on admet que l'oeil satisfait â l'hypothèse d'addivité spectrale, c'est-à-dire que la stimulation visuelle d'une source à spectre étendu résulte en la sommation des stimulations visuelles élémentaires obtenues par la décomposition de son spectre d'émission en contributions quasi monochromatiques : le flux lumineux élémentaire émis par la source entre les longueurs d'onde $\lambda$ et $\lambda+d \lambda$ est, d'après les définitions ci-dessus, lié au flux énergétique $\mathrm{dF}_{\mathrm{e}}$ dans la même bande par le coefficient de transfert (par exemple, $K_{m} V(\lambda)$ en vision photopique) :

$$
d F_{v}=K_{m} V(\lambda) d F_{e}=K_{m} V(\lambda)\left[\frac{d F_{e}}{d a}\right] d a=\left[\frac{d F_{v}}{d a}\right] d a
$$

Le flux lumineux global de la source est alors:

$$
F_{v}=\int_{0}^{\infty} d F_{v}=K_{m} \int_{0}^{\infty} v(\lambda)\left(\frac{d F_{0}}{d a}\right) d a
$$

Puisque d'autre part, le flux énergétique global de la source est défini par :

$$
F_{\theta}==\int_{0}^{\infty}\left(\frac{d F_{\theta}}{d a}\right) d a
$$


Le rapport entre les flux lumineux et énergétique émis par une source ne dépend que de la distribution spectrale $S(\lambda)$ de la source :

Mathématiquement, l'intégration du numérateur s'effectue entre $\lambda \sim 0,4 \mu \mathrm{m}$ et $\lambda \sim 0,8 \mu \mathrm{m}$ puisque la fonction $V(\lambda) \sim 0$ en dehors de ce domaine.

Puisque les relations entre flux, luminance, intensité, éclairement caractéristiques d'un rayonnement donné ne dépendent que des propriétés géométriques de ce rayonnement, on peut écrire que :

$$
F_{v} / F_{Q}=h_{v} / I_{\theta}=L_{v} / L_{e}=E_{v} / E_{\theta}=\eta(I m / W)
$$

oủ $\eta$ est refficacité lumineuse du rayonnement, paramètre très important dans tous les problèmes d'éclairage. D'après ce qui a été vu ci- dessus, les sources les plus efficaces sont (en vision photopique. la plus courante à traiter) celles qui émettent un rayonnement quasi monochromatique à $\lambda=0,555 \mu \mathrm{m}$. pour lesquelles $\eta=683 \mathrm{Im} / \mathrm{W}$. Pour toute autre source, monochromatique à $\lambda=0,555 \mu \mathrm{m}$ ou à spectre étendu, il est évident que $\eta<683 \mathrm{Im} / \mathrm{W}$.

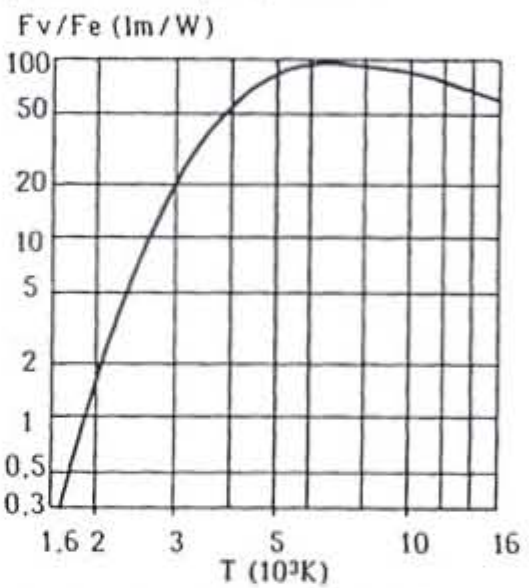

Fig III-3 : Efficacité lumineuse du Corps Noir en fonction de sa température
Pour illustrer cette notion d'efficacité lumineuse de source à spectre large, on donne (Fig III-3) son évolution pour les Corps Noirs en fonction de leur temperature :

La courbe obtenue montre que le maximum d'efficacité lumineuse du Corps Noir ( $90 \mathrm{Im} /$ ) s'obtient pour une température de l'ordre de 6000 K(soleil).

$$
\begin{aligned}
\eta_{C N}(T)=L_{V} / L_{\theta} & =K_{m} \frac{\int\left(\frac{d L}{d \lambda}\right)_{C N}^{T} V(\lambda) d \lambda}{\int\left(\frac{d L}{d \lambda}\right)_{C N}^{T} d \lambda} \\
& =\frac{K_{m}}{K_{3} T^{4}} \int_{0}^{T}\left[\frac{d L}{d \lambda}\right]_{C N}^{T} V(\lambda) d \lambda
\end{aligned}
$$

\section{III.2. PARAMETRES DE SIGNAL D'UN DETECTEUR PHYSIQUE (Détection directe)}

Un détecteur physique est un matériau ou composant opto-électronique qui, sous l'effet d'un rayonnement qu'il absorbe au moins partiellement, foumit un signal, généralement électrique. Traditionnellement, on distingue 2 familles essentielles de détecteurs :

- d'une part, les détecteurs dits "thermiques" dans lesquels l'énergie du rayonnement absorbé se transforme en chaleur. Dans la mesure où le facteur d'absorption spectral du matériau est neutre, le signal fourni est proportionnel au flux énergétique incident $F_{0}$.

- D'autre part, les détecteurs dits "quantiques" ou "photoniques" dans lesquels l'absorption de chaque photon incident donne lieu (avec une probabilité < 1) à la création d'une charge électrique récupérable. Le signal foumi est dans ce cas proportionnel au flux photonique $F_{p}$ absorbé par le matériau.

Parmi les paramètres utilisés pour caractériser le comportement đoun détecteur, on séparera ceux qui permettent de calculer le signal délivré par le détecteur ou "paramètres de signal" de ceux qui imposent une limite au bon fonctionnement du détecteur, ou "paramètres de bruit". Ce paragraphe traite des paramètres de signal essentiels des détecteurs physiques tels que facteur de réponse et bande passante. 


\section{III.2.1. FACTEUR DE REPONSE SPECTRALE}

Par définition, le facteur de réponse (ou sensibilité) spectrale d'un détecteur est égal, à la longueur d'onde $\lambda$, au rapport entre le signal délivré par le détecteur et le flux énergétique incident $F_{0}$. pour un rayonnement quasi monochromatique à cette longueur d'onde.

On définit ainsi les facteurs de réponse spectrale en courant, $\varkappa_{i}(\lambda)$ ou en tension, $\varkappa_{v}(\lambda)$ :

$$
R_{i}(\lambda)=i S / F_{\theta}(\lambda) \text {, en } S_{w} \quad R_{\theta}(\lambda)=v S / F_{\theta}(\lambda) \text {, en } v / w
$$

Dans le cas des détecteurs thermiques, qui délivrent un signal (is ou $\mathrm{v}_{\mathbf{s}}$ ) proportionnel au flux énergétique incident absorbé, le facteur de réponse spectral est uniforme sur le domaine où le facteur d'absorption spectral du matériau est constant.

Pour les détecteurs photoniques, l'absorption d'un photon incident ne peut se produire que si son énergie est supérieure à un seuil $\Delta \mathrm{E}$, défini par la différence énergétique entre les niveaux permis du matériau. La probabilité d'absorption d'un photon est théoriquement nulle, pour des rayonnements de longueur d'onde supérieures à la longueur d'onde "de coupure" $\lambda_{c}$ correspondante $\left(\lambda_{c}=h c / \Delta E\right)$ et par conséquent, la réponse du détecteur est nulle pour $\lambda>\lambda_{c}$.

Pour les rayonnements de longueurs d'onde plus faibles, chaque photon incident entraine la création d'une charge avec une probabilité $\eta(\lambda)$ et l'ensemble du flux photonique $F_{p}(\lambda)$ créé en sortie de détecteur un courant de signal is tel que :

$$
\text { is }=\eta(\lambda) F_{\rho}(\lambda) e=\frac{\eta(\lambda) e}{h_{N}} F_{e}(\lambda)
$$

Il en résulte que le facteur de réponse spectral en courant d'un détacteur photonique est :

$$
\begin{aligned}
& R_{i}(\lambda)=\frac{\eta(\lambda) e}{h N}=\frac{\eta(\lambda) e}{h c} \lambda \text { pour } \lambda<\lambda_{c} \\
& R_{i}(\lambda)=0 \text { pour } \lambda>\lambda_{c}
\end{aligned}
$$

Le graphe de la Figure III-4 ci-dessous représente rallure théorique d'une courbe de facteur de réponse spectral d'un détecteur photonique à rendement quantique uniforme. (cou r be $a$ ).

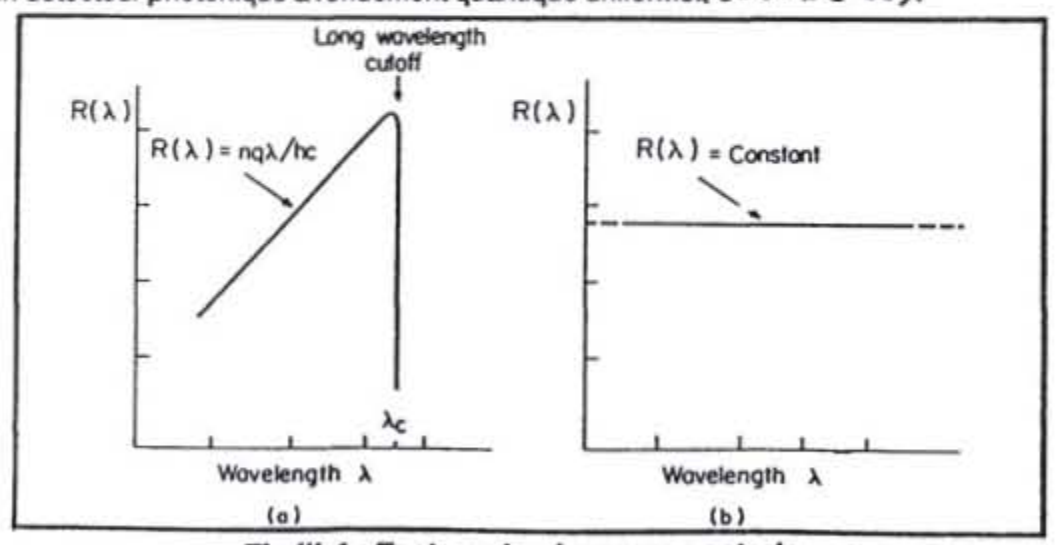

Fig III-4 : Facteur de réponse spectrale 
Le schéma de la Figure III-5 représente un montage typique utilisé pour mesurer le facteur de réponse spectral d'un détecteur : une source à spectre large éclaire la fente d'entrée d'un monochromateur (appareil destiné à isoler et transmettre une portion étroite du spectre émis par la source, et à lui faire "balayer" le domaine spectral de fonctionnement du détecteur à caractériser). Le flux quasi monochromatique en sortie du monochromateur, généralement modulé en amplitude grâce à un modulateur ou "chopper" (pour fins de filtrage du signal électronique et amélioration de la qualité des mesures), est envoyé successivement sur le détecteur sous test et sur un détecteur de référence connu (à réponse uniforme). La comparaison des signaux issus de ces 2 détecteurs en fonction de la longueur d'onde du rayonnement permet de déduire le facteur de réponse spectrale recherché.

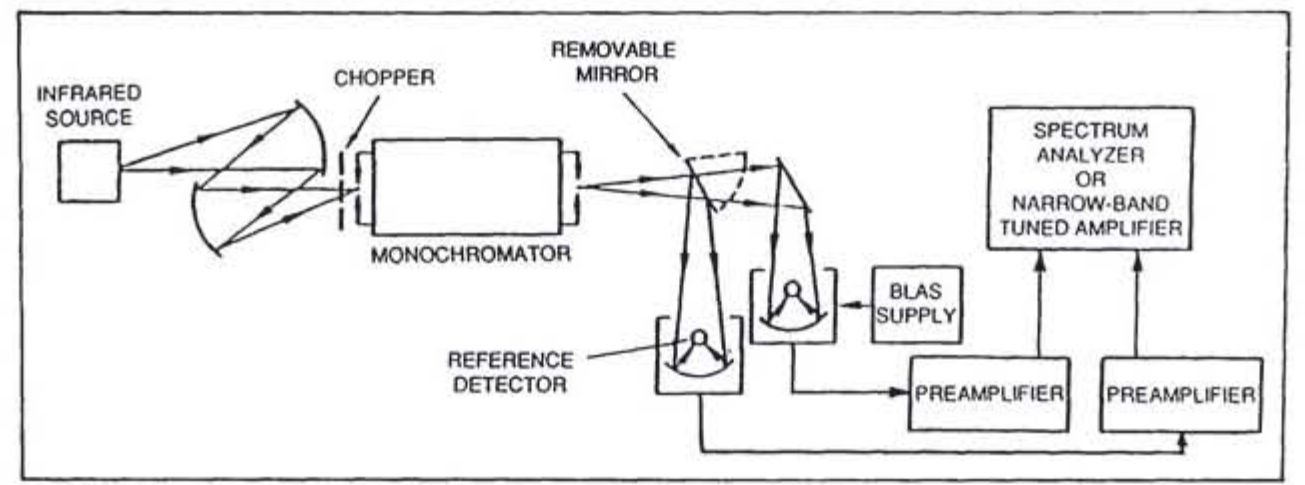

Fig III-5 : Mesure expérimentale de facteur de réponse spectrale de détecteur

On définit aussi un facteur de réponse dit "au corps noir" $R\left(T_{C N}\right)$ pour caractériser le comportement global du détecteur face à une source à spectre large (par exemple Corps Noir à la température TCN). Le montage expérimental, pour cette mesure est simple (il ne comprend qu'un Corps Noir et un modulateur) et la comparaison avec la théorie peut être rapidement faite puisque :

$$
R_{S}(T C N)=i_{S} / G K_{3} T_{C N}^{4}
$$

où G est l'étendue géométrique définie par la surface émissive du Corps Noir et l'aire du détecteur.

\section{III.2.2. BANDE PASSANTE}

Cette caractéristique concerne les variations du facteur de réponse du détecteur (pour une tension de polarisation fixe), muni de lélectronique associée, en fonction de la fréquence de modulation du flux incident.

Elle se mesure habituellement par la valeur du taux de modulation du signal de sortie pour une modulation de $100 \%$ du flux à l'entrée. La fréquence pour laquelle la modulation est égale à 1/N2 fois sa valeur à basse fréquence est appelée fréquence de coupure à $3 \mathrm{~dB}$.

Une autre méthode de mesure consiste à faire subir au détecteur une variation d'éclairement très rapide (fonction "escalier" ou de Heaviside).

Le temps de réponse $\tau$ du détecteur (temps mis par le signal pour atteindre $75 \%$ de sa valeur asymptotique) est lié à sa fréquence de coupure par la relation :

$$
\tau=1 / 2 \pi f_{3} d B
$$


et la décroissance du facteur de réponse en fonction de la fréquence du signal incident est donnée par :

$$
\mathcal{R}(f)=\mathcal{R}(0) /\left(1+4 \pi^{2} f^{2} \tau^{2}\right)^{1 / 2}
$$

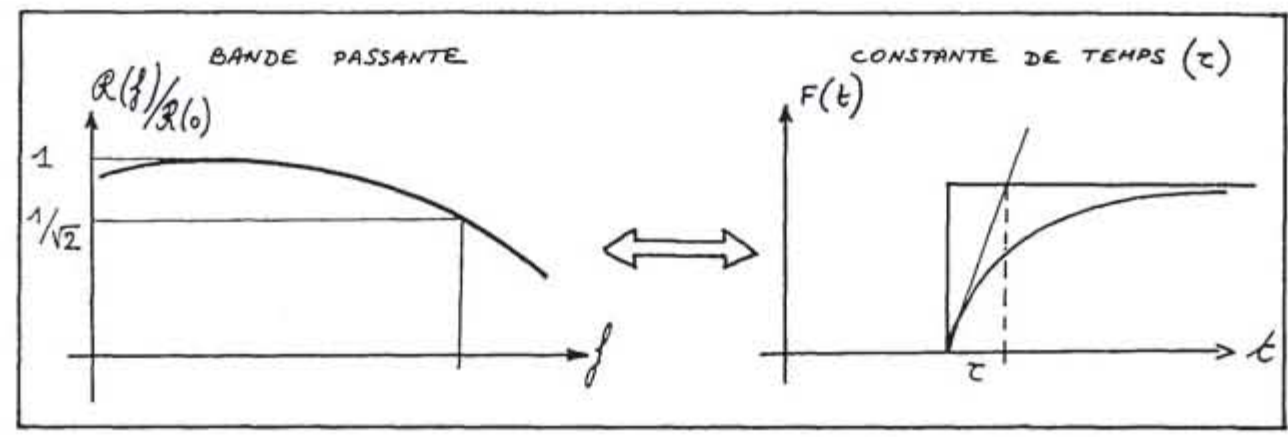

Fig III-6: Bande passante et temps de réponse d'un détecteur

\section{III.3. DETECTIVITE D'UN DETECTEUR (cas de la détection directe)}

La connaissance du facteur de réponse d'un détecteur n'est pas suffisante pour en déduire les performances ultimes du capteur, par exemple ses limites de fonctionnement en détection, qui se trouvent définies par des perturbations (ou bruit) propres au détecteur considéré, à son environnement et à la qualité de mise en oeuvre. Ce paragraphe rappelle les sources de bruit dans un récepteur optique et présente les notions de base dans l'évaluation des períormances d'un détecteur, telles que "flux équivalent au bruit" et "détectivité".

\section{III.3.1. FLUX EQUIVALENT AU BRUIT}

Les photoélectrons fournis par le détecteur en réponse aux photons incidents sont exploités par l'électronique de traitement sous forme de courant dans une impédance de charge (résistance Rc) ou de tension à ses bornes.

En l'absence de tout rayonnement utile sur le détecteur, on constate que ce signal électrique au niveau de la résistance de charge (courant ib ou tension vo "dits" de bruit), subit des fluctuations temporelles qui définissent une puissance de bruit $\mathrm{Pb}_{\mathrm{b}}$ égale à :

$$
P_{b}=R_{c} i_{b}^{2}=v_{b}^{2} / R_{c}
$$

ou ib ${ }^{2}$ et $v_{0}^{2}$ sont les variances respectives du courant et de la tension de bruit pour une bande passante de mesure égale à $\mathrm{B}$.

Par définition, le flux équivalent au bruit du détecteur (incluant son préamplificateur ou de façon flus générale, son électronique de proximité), dénommé FEB ou, plus communément NEP (Noise Equivalent Power) est la valeur du flux de signal en entrée de détecteur pour laquelle le signal électrique résultant dans la résistance de charge est égal à la valeur efficace du bruit mesurée au niveau de cette résistance. Puisque le facteur de réponse du détecteur est une variable fonction de la longueur d'onde, on définit un flux équivalent au bruit quasi monochromatique NEP $(\lambda)$, tel que :

$$
\left(i_{b}^{2}\right)^{1 / 2}=R_{i}(\lambda) \operatorname{NEP}(\lambda)
$$




\section{III.3.2. SOURCES DE BRUIT DANS UNE RESISTANCE DE CHARGE}

Pour évaluer le bruit électrique dans la résistance de charge du détecteur, on rappelle tout d'abord qu'il existe deux mécanismes fondamentaux générateurs de bruit dans une résistance :

- Le bruit Shot (ou Schottky ou de grenaille):

Si une résistance est traversée par un courant électrique de valeur moyenne $\mathrm{i}$, le caractère discret des charges (électrons) qu'il transporte induit des fluctuations autour de cette valeur moyenne, qui obéissent à la statistique de Poisson et sont telles que :

$$
\left[i b^{2}\right]_{\text {shot }}=2 e \overline{T B}
$$

- Le bruit thermique (ou Johnson):

En l'absence de tout courant, les mouvements aléatoires des charges dus à la température de la résistance induisent des fluctuations de signal électrique exprimées par :

$$
\left.\left[\overrightarrow{i b}^{2}\right\}\right] \text { Johnson }=4 k T B / R_{C}
$$

où $\mathrm{k}=$ constante de Boltzmann

$T=$ température de la résistance

Ainsi, la résistance de charge du détecteur est le siège de bruits électriques, provenant des divers courants qui la traversent et de sa température propre. Parmi ces courants, qui proviennent du détecteur même en l'absence du rayonnement utile, on mentionnera essentiellement le courant de fond, if, créé par le détecteur en réponse au rayonnement ambiant, le courant d'obscurité, io, généralement courant de fuite, provenant des circuits de polarisation. II faut de plus tenir compte du bruit propre aux circuits de préamplification que l'on ramène en amont par le calcul (au niveau de la résistance de charge), grâce à leur facteur de bruit $F$. Le tableau ci-dessous résume les différents types de bruit dans la résistance, avec leurs expressions respectives :

\begin{tabular}{|c|c|c|}
\hline SOURCE DE BRUIT & PARAMETRE & $i \frac{2}{6}$ \\
\hline SIGNAL & COURANT DE SIGNAL ${ }^{i_{S}}$ & $2 \mathrm{e} \mathrm{i}_{\mathrm{s}} \mathrm{B}$ \\
\hline FOND & COURANT DE FOND $i_{F}$ & 2 e $i_{F} B$ \\
\hline DETECTEUR & COURANT D'OBSCURITE ${ }^{i}{ }_{0}$ & $2 \mathrm{e} \mathrm{i}_{0} \mathrm{~B}$ \\
\hline OSCILLATEUR LOCAL & COURANT D'OSC. LOCAL IOL & 2 e $i_{\Delta L}^{B}$ \\
\hline JOHNSON (THERMIQUE) & TEMPERATURE T DE $R_{c}$ & $4 \mathrm{k} \quad \mathrm{T} \quad \mathrm{B} / \mathrm{R}_{\mathrm{C}}$ \\
\hline AMPLIFICATEUR & FACTEUR DE BRUIT F & $4 k(F-1) T B / R_{C}$ \\
\hline DETECTEUR $(1 / f)$ & SPECIFIQUE AU DETECTEUR & A DEFINIR \\
\hline
\end{tabular}

Tableau III-2 : Sources de bruit dans la résistance de cha.ge d'un dé,ecteur

Puisque ces diverses sources de bruit sont décorrélées l'une de l'autre, la variance de bruit global dans la résistance s'exprime généralement sous la forme :

$$
\left[\overrightarrow{i b}^{2}\right]=2 e\left[\sum_{n}^{1} 7\right] B+4 k F T B / R_{C}
$$


Suivant les domaines de fonctionnement spectral et fréquentiel du détecteur, l'une des sources de bruit sera prépondérante vis à vis des autres : par exemple, si la fréquence de fonctionnement du détecteur est supérieure à quelques $\mathrm{kHz}$, on négligera le bruit en $1 / f$. Dans le cas de détecteurs fonctionnant dans le visible, le proche infrarouge ou l'infrarouge très lointain, le bruit Johnson l'emporte généralement sur le bruit shot. Par contre, dans l'infrarouge compris entre $3 \mu \mathrm{m}$ et $30 \mu \mathrm{m}$, et pour une température de fond de l'ordre de $300 \mathrm{~K}$, le nombre de photons ambiants incidents sur le détecteur est très important, et c'est le bruit dû au fond environnant (courant de fond) qui l'emporte sur les autres sources de bruit : on se trouve alors en présence de détecteurs "BLIP" (Background Limited Infrared Photodetetors).

\section{III.3.3. DETECTIVITE SPECTRALE D'UN DETECTEUR BLIP}

Pour caractériser les détecteurs grâce à un paramètre dont la valeur soit proportionnelle à sa qualité en détectabilité, les fabricants de détecteurs ont introduit la notion de "détectivité spectrale" dont la valeur est linverse du NEP( $\lambda)$ qui, lui, est d'autant plus faible que le détecteur est performant) :

$$
D(\lambda)=1 / N E P(\lambda)
$$

Dans le cas d'un détecteur dit BLIP, donc limité par le bruit dô au fond, la variance du courant de bruit ib ${ }^{2}$ est :

$$
i b^{2}=2 \text { if } B
$$

D'après le paragraphe III.3.1, le flux équivalent au bruit à la longueur d'onde $\lambda$ est :

$$
\begin{aligned}
\operatorname{NEP}(\lambda) & =\left(2 e i_{i F} B\right)^{1 / 2} / \Re_{i}(\lambda) \\
& =h c(2 e i F B)^{1 / 2} / \eta e \lambda
\end{aligned}
$$

Le courant de fond fourni par le détecteur est proportionnel au flux de photons "actifs" (c'est-á-dire de longueur d'onde $\lambda_{c}$ ) provenant de l'environnement et au rendement quantique du détecteur (supposé constant pour $\left.\lambda<\lambda_{c}\right)$ :

$$
\mathrm{iF}=\eta \in\left[\mathrm{Ep}, \mathrm{f}^{\lambda \mathrm{C}} \mathrm{Ad}\right.
$$

où $\left[E p, \eta_{0}^{\lambda c}\right.$ est l'éclairement photonique du detecteur en provenance du fond entre $\lambda=0$ et $\lambda=\lambda_{c}$ et $A_{d}$ l'aire sensible du détecteur.

On rappelle d'autre part que si le fond est observé par le détecteur sous un cône de demi-angle au sommet $\theta_{\mathrm{M}}$ avec une iuminance photonique uniforme $\iota_{p}, f$, son éclairement photonique est donné par l'expression (paragraphe 1.2.5):

$$
E p, f=\pi L p, f \sin ^{2} \theta M
$$

II ressort des relations précédentes que la détectivité spectrale d'un détecteur photonique de qualité BLIP est égale à :

$$
\begin{aligned}
D(\lambda)=\frac{1}{h c} \frac{A_{d}^{-1 / 2}}{\sin \theta M} & {\left[\frac{\eta}{2 \pi\left[L_{0,} f\right]_{0}^{\lambda c}}\right]^{1 / 2} B^{-1 / 2} \lambda \text { pour } \lambda<\lambda_{c} } \\
& =0 \text { pour } \lambda>\lambda_{c}
\end{aligned}
$$

Dans un souci de normalisation dans la métrologie des détecteurs et d'équité dans la comparaison entre détecteurs, la notion de "détectivité spécifique spectiale" notée $D^{\circ}(\lambda)$ a été introduite, qui représente la détectivité spectrale d'un détecteur par unité de surface (Attention: $A d=1 \mathrm{~cm}^{2}$ ) et de bande passante de mesure $(B=1 \mathrm{~Hz})$. 
On peut donc écrire que :

$$
\begin{gathered}
D^{*}(\lambda)=D(\lambda) \sqrt{A_{d}} \sqrt{B} \\
\text { donc écrire que : } \quad N E P(\lambda)=\frac{\sqrt{A_{d}} \sqrt{B}}{D^{*}(\lambda)} \\
D^{*}(\lambda)=\frac{1}{h c} \frac{1}{\sin \theta M}\left[\frac{\eta}{2 \pi\left[L_{0, t}\right]_{c}}\right]_{0}^{1 / 2} \lambda \quad \text { pour } \lambda<\lambda_{c}\left(D^{*}(\lambda)=0 \text { si } \lambda>\lambda_{c}\right)
\end{gathered}
$$

Les paramètres qui entrent dans la définition de la détectivité spécifique spectrale d'un détecteur BLIP sont pour un matériau donné : l'ouverture $\theta_{M}$ du cône de réception, la luminance photonique du fond vu par le détecteur (et donc, sa température), le rendement quantique du détecteur $(\eta)$, la longueur d'onde de coupure définie par le "gap" du matériau $\left(\lambda_{c}\right)$ et la fréquence de modulation du signal incident.

Le tableau III-3 rappelle les formules du rayonnement du Corps Noir dans le système d'unités photoniques, directement utilisable pour exprimer la détectivité spécifique spectrale d'un détecteur BLIP. Le graphe de la Figure III-7 représente la valeur de l'exitance photonique du Corps Noir comprise entre $\lambda=0$ et $\lambda=\lambda_{c}$ en fonction de la longueur d'onde de coupure du détecteur $\lambda_{c}$.

Tableau III-3 : Rayonnement du Corps Noir (unités photoniques)

\begin{tabular}{|c|c|}
\hline QUANTITE (loi) & Photonique \\
\hline $\begin{array}{c}\text { Luminance } \\
\text { spectrique } \\
\text { (Planck) }\end{array}$ & $\begin{array}{c}{\left[d L_{\rho} / d a\right]_{C N}^{T}} \\
2 c \lambda^{4}\left[\frac{h c}{\theta \lambda k T}-1\right]\end{array}$ \\
\hline $\begin{array}{c}\text { Coordonnées du } \\
\text { maximum d'émission }\end{array}$ & $\begin{array}{l}\lambda_{m} T=3670 \mu m K \\
{\left[d L_{\rho} / d a\right] M=K_{2}^{\prime} T^{4}}\end{array}$ \\
\hline Luminance totale & $L_{p}=K_{3} T^{3}$ \\
\hline
\end{tabular}

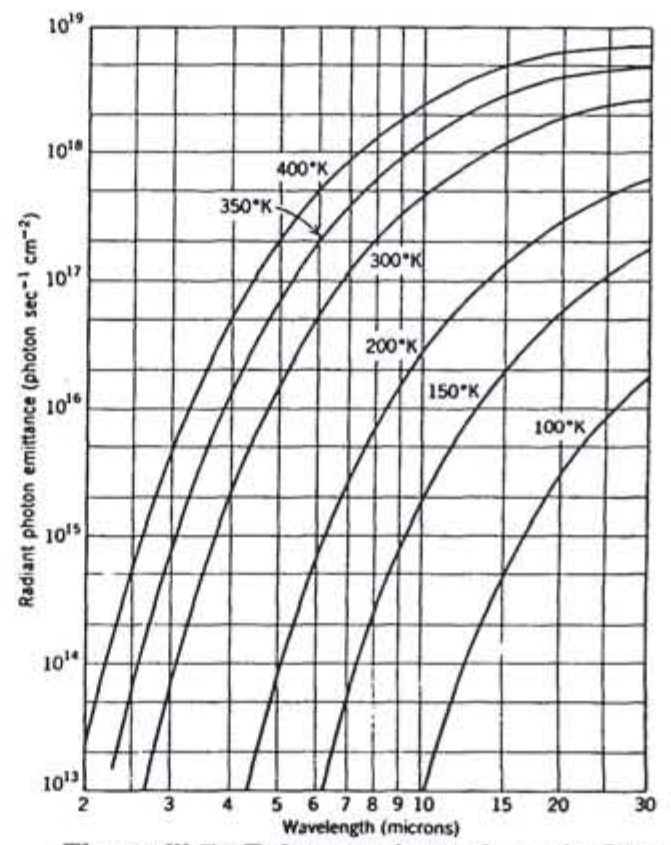

Figure III-7 : Exitance photonique du Corps Noir entre $\lambda=0$ et $\lambda=\lambda c$

L'expression mathématique de $D^{*}(\lambda)$ couplée aux résultats de la Figure III-7, permet d'aboutir à la loi (Figure III-8) de variation de la détectivité spécifique spectrale au pic (pour $\lambda=\lambda_{c}$ ) pour un détecteur BLIP parfait $(\eta=1)$ observant sous un angle solide de $2 \pi s r(\theta M=\pi / 2)$ un fond equivalent à un corps noir de temperature $\mathrm{Tf}(\mathrm{Tf}=295 \mathrm{~K})$. En pratique, cette loi est donnée sous forme de 2 courbes, l'une correspondant aux détecteurs dits "photovoltaīques", étant V2 fois supérieure à la seconde correspondant aux "photoconducteurs", ces demiers délivrant pour un meme fond 2 fois plus de charges que les premiers. La figure III-8 montre aussi les courbes de $D^{\circ}(\lambda)$ pour différents détecteurs courants. 


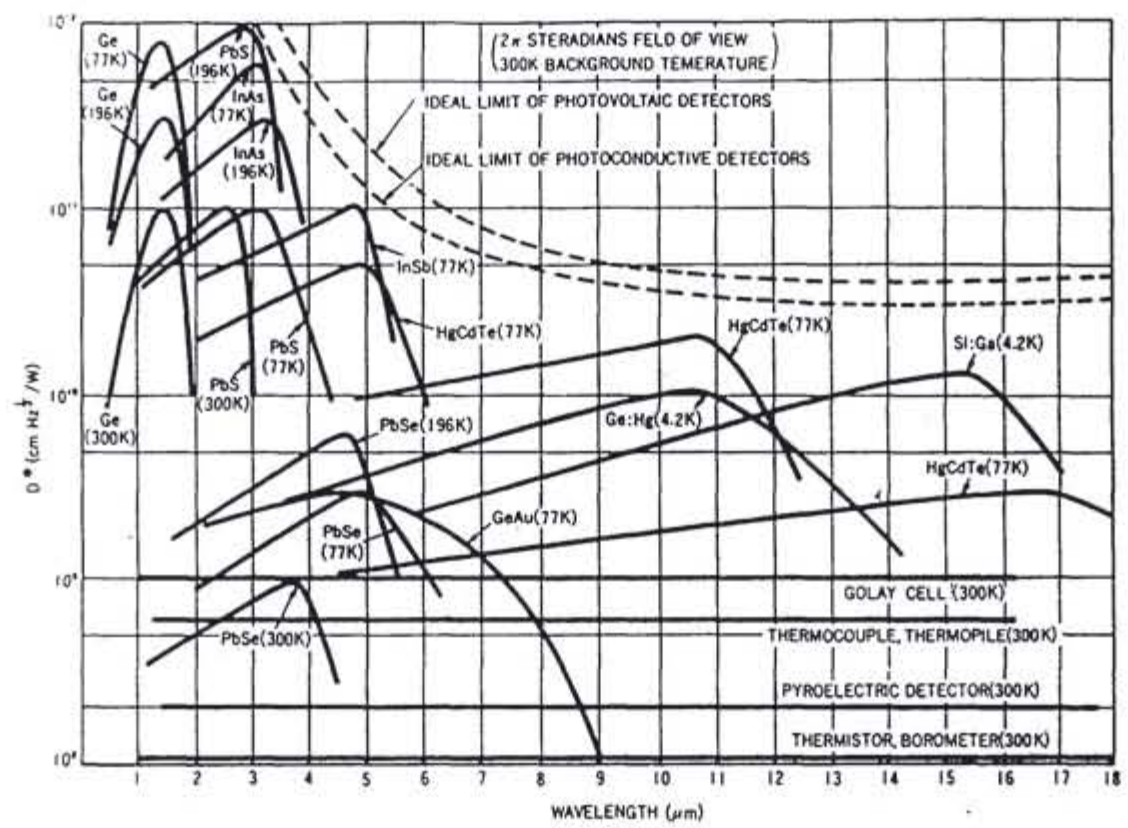

Fig III-8 : Détectivités spectrales spécifiques de détecteurs IR

\section{III.4. CAS DE LA DETECTION HETERODYNE (ou cohérente)}

D'après le paragraphe 1.1.2, un détecteur optique est dit quadratique, c'est-à-dire que son signal est proportionnel au carré du champ électrique présent sur la surface sensible. Lorsque le détecteur est utilisé dans le mode "direct", le seul champ électrique présent sur le détecteur est celui du rayonnement à détecter. Dans le cas d'un flux quasi monochromatique, le vecteur de Poynting résultant (représentatif du carré du champ E.M.) oscille à une fréquence double de celle du champ optique ( $>1$ Térahertz), donc très supérieure à la bande passante du détecteur qui délivre alors un courant proportionnel à la moyenne du carré du champ:

$$
i(t)=\frac{\eta e}{h_{N}} \frac{A_{d}}{Z_{0}}<E^{2}(t)>=\frac{1}{2} \frac{\eta e}{h_{N}} \frac{A_{d}}{Z_{0}}\left|E_{0}\right|^{2}=\frac{\eta e}{h_{N}} F
$$

oùl Eol est l'amplitude du champ électrique.

Dans le montage de détection hétérodyne de la Figure III-9 (technique de détection très utilisée dans les domaines radio ou radar), l'onde à détecter que l'on supposera monochromatique et polarisée linéairement, interfère sur la surface sensible du détecteur avec une onde de référence de même polarisation et de fréquence voisine, appelée oscillateur local.

Si les champs électriques de ces 2 ondes sont respectivement :

$$
\begin{aligned}
& \overrightarrow{E_{S}}(t)=\overrightarrow{E S} \exp [-j(\omega s t+\varphi S)] \text { pour le signal } \\
& \overrightarrow{E O L}(t)=\overrightarrow{E O L} \exp \left[-j(\omega O L t] \quad \text { pour } l^{\prime} O L\right.
\end{aligned}
$$

le champ résultant à la surface du détecteur est alors:

$$
\vec{E}(t)=\overrightarrow{E_{S}}(t)+\overrightarrow{E_{O L}}(t)
$$


Dans l'hypothèse (simplificatrice) où ces 2 champs sont uniformes et leur déphasage relatif constant sur tout le détecteur (dont le rendement est supposé uniforme), le courant délivré par le détecteur s'exprime par:

$$
i(t)=\frac{\eta e A_{d}}{h v Z_{0}} I E(t) I^{2}
$$

Le développement mathématique de cette expression fait apparaître 4 termes, dont on retiendra la signification des 3 suivants :

$$
\begin{aligned}
& i O L=\frac{\eta e}{h N} F O L \quad \begin{array}{l}
\text { Courant que délivrerait le détecteur en présence de l'onde } O L \text { seule, dit courant } \\
\text { d'oscillateur local) }
\end{array} \\
& i s=\frac{\eta e}{h_{N}} F S \quad \begin{array}{l}
\text { Courant que delivrerait le détecteur en présence du signal optique seul, donc en } \\
\text { mode direct }(\text { is }<<i o L)
\end{array} \\
& i_{H E T}=\frac{2 \eta e}{h N} \sqrt{F O L F S} \cos [(\omega S-\omega O L) t+\varphi S] \quad \text { Courant de signal dit hétérodyne. }
\end{aligned}
$$

Le niveau de l'oscillateur local sur le détecteur est réglé à une valeur suffisamment élevée pour que le bruit électrique dans la résistance de charge du détecteur (voir paragraphe III.3.2) soit imposé majoritairement par le bruit shot dû au courant d'oscillateur local, ce qui entraîne l'approximation suivante :

$$
i b^{2}=2 \text { e iOL } B=2 \eta \frac{e^{2} F O L}{h v} B
$$

D'autre part, la puissance électrique du signal hétérodyne est proportionnelle à :

$$
\begin{aligned}
\text { IHET } & =\frac{4 \eta^{2} e^{2}}{h^{2} v^{2}} \sqrt{F O L F S}<\cos ^{2}[(\omega S-\omega O L) t+\varphi S]> \\
& =2 \frac{\eta^{2} e^{2}}{h^{2} v^{2}} \text { FOL FS }
\end{aligned}
$$

Le flux équivalent au bruit d'un détecteur utilisé en mode hétérodyne, obtenu par l'égalité entre courant đe bruit et courant de signal hétérodyne est donc égal à :

$$
\text { NEPHET }(\lambda)=\frac{h v}{\eta} B
$$

Ce résultat est à comparer avec la valeur correspondante en détection directe :

pour le choix du mode de détection optimal.

$$
\operatorname{NEP} D \text { IR }(\lambda)=\sqrt{A_{d}} \sqrt{B} / D^{*}(\lambda)
$$

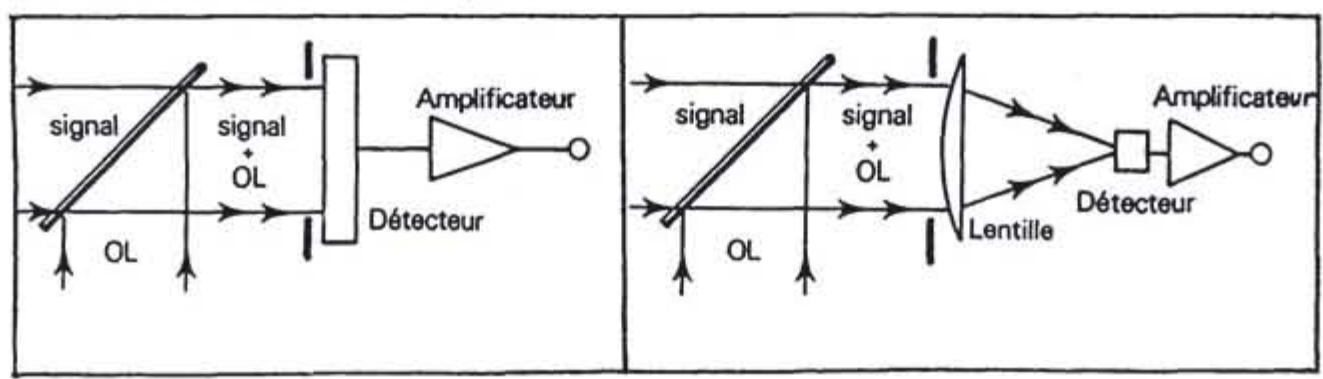

Fig 9: Montages de détection hétérodyne 


\section{III.5. EXEMPLES DE DETECTEURS THERMIQUES.}

Dans un détecteur thermique, l'absorption du rayonnement à détecter se traduit en chaleur et l'élévation de température résultante est convertie en signal électrique. Les variations spectrales du facteur de réponse $T(\lambda)$ ne dépendent que de celles du facteur d'absorption $\lambda(\lambda)$ du revêtement absorbant déposé ensurface (certains revêtements, comme la suie d'or, ont un facteur d'absorption proche de 1 depuis l'ultraviolet jusqu'à l'infrarouge relativement lointain.)

Leurs détectivités sont faibles vis à vis de celles des détecteurs photoniques et ils sont lents (temps de réponse typiquement de quelques ms), à l'exception des pyroélectriques. Par contre, ils présentent les avantages d'un fonctionnement à température ambiante et d'une grande uniformité spectrale sur des domaines étendus en longueur d'onde. Ils s'utilisent dans des équipements embarqués sur satellites ou en détection d'intrusion, mais surtout en laboratoire et métrologie (radiomètrie énergétique, calorimétrie laser, spectrophotométrie, ...). Parmi les détecteurs thermiques les plus usités, on citera les thermopiles. les bolomètres et les pyroélectriques.

\section{III.5.1. THERMOPILES}

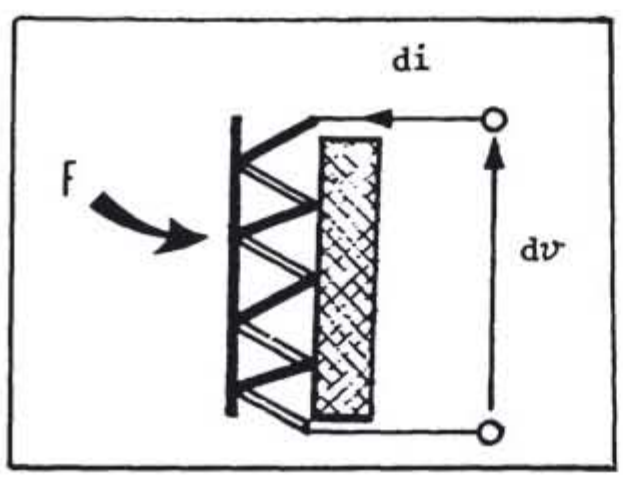

Fig III-10: Thermopiles
Une thermopile est constituée d'une chaîne de thermocouples, soudures produisant une d.d.p. par effet thermoélectrique (effet Seebeck) sous l'effet d'une différence de température) (Figure III-10). Les soudures "chaudes" sont en contact avec l'absorbeur qui élève leur température par rapport à l'ambiante sous l'effet du rayonnement incident et les soudures "froides" maintenues à la température ambiante par contact avec une masse à forte inertie thermique.

La mise en série de plusieurs thermocouples permet de recueillir une tension proportionnelle à leur nombre. Le facteur de réponse de tels dispositifs va de quelques $V / W$ à quelques dizaines de $V M$ et leur temps de réponse de quelques 10 à $100 \mathrm{~ms}$. Les thermopiles fonctionnent sans polarisation.

\section{III.5.2. BOLOMETRES}

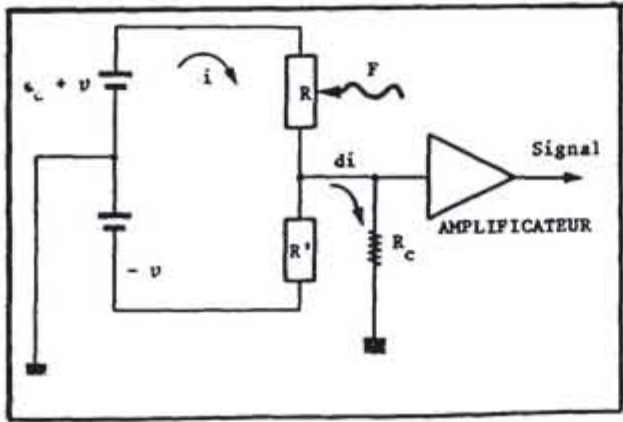

Les bolomètres sont des détecteurs thermiques comportant un matériau dont la conductivité varie avec la température. Pour observer cette variation de résistance, il est nécessaire d'appliquer une tension aux composants. Pour éviter les dérives en courant provenant des variations de température ambiante, un bolomètre utilise généralement 2 éléments identiques, dont l'un est exposé au rayonnement à détecter et l'autre protégé de ce rayonnement ; la variation de résistance entre ces 2 éléments essentiellement due au flux de signal recherché est alors mesurée dans un montage à pont de Wheatstone (Figure III-11)

Fig III-11 : Bolomètres 
Les matériaux employés sont des métaux ou des semi-conducteurs (thermistances). A très basse température, la supraconductivité et la faiblesse de l'agitation thermique des électrons conduisent à la réalisation de détecteurs dans l'infrarouge sensibles et rapides.

\section{III.5.3. DETECTEURS PYROELECTRIQUES}

L'effet pyroélectrique se manifeste dans les cristaux isolants dont la maille ne présente pas de centre de symétrie. II consiste en un déplacement global des électrons de liaison par rapport au réseau cristallin en fonction de la température. Si l'on dépose 2 électrodes sur les faces d'une lame d'un matériau pyroélectrique perpendiculairement à la direction de ce déplacement, ces dernières préleveront les charges qui se seront déplacées à chaque variation de température du crystal soumis à un éclairement variable.

II faut noter que le signal électrique recueilli (courant) est proportionnel à la variation dans le temps du nombre de charges collectées par les électrodes. II est donc nul si le flux incident sur le matériau est constant. Ces détecteurs ne peuvent donc s'utiliser que pour détecter des rayonnements modulés en fonction du temps. lls constituent les plus rapides des détecteurs thermiques.

Les matériaux utilisés pour la constitution des détecteurs pyroélectriques sont le sulfate de glycocolle (TGS), le titanate de Baryum $\left(\mathrm{BaTiO}_{4}\right)$, le tantalate de Lithium $\left(\mathrm{LTaO}_{3}\right)$. lls se présentent généralement sous la forme de détecteurs élémentaires, avec des surfaces sensibles de l'ordre de 1 à quelques mm2, sur un domaine spectral de 2 à $35 \mu \mathrm{m}$. lls peuvent aussi se montrer dans des tubes à imagerie infrarouge ("pyricon") : ils y présentent lintérêt de ne pas nécessiter de système de refroidissement, mais par contre leur détectivité demeure beaucoup plus faible que celle des détecteurs quantiques, ils sont microphoniques et nécessitent l'emploi de modulateurs de lumière.

\section{III.5.4. UTILISATION EN CALORIMETRIE LASER}

A cause de leur uniformité en réponse spectrale, de leur robustesse et longévité, les détecteurs thermiques sont le plus souvent utilisés en métrologie, calorimétrie (en particulier pour lasers) ou dans les applications spatiales.

Par exemple, le montage de la Figure III-12 illustre lutilisation d'un calorimètre laser pour la caractérisation d'une source laser impulsionnelle : une lame séparatrice placée en sortie de laser prélève une faible fraction du flux pour la diriger sur un détecteur photonique "rapide", non étalonné, chargé de relever la caractéristique temporelle de l'impulsion laser, par exemple, sa largeur à mi-hauteur $\tau$.

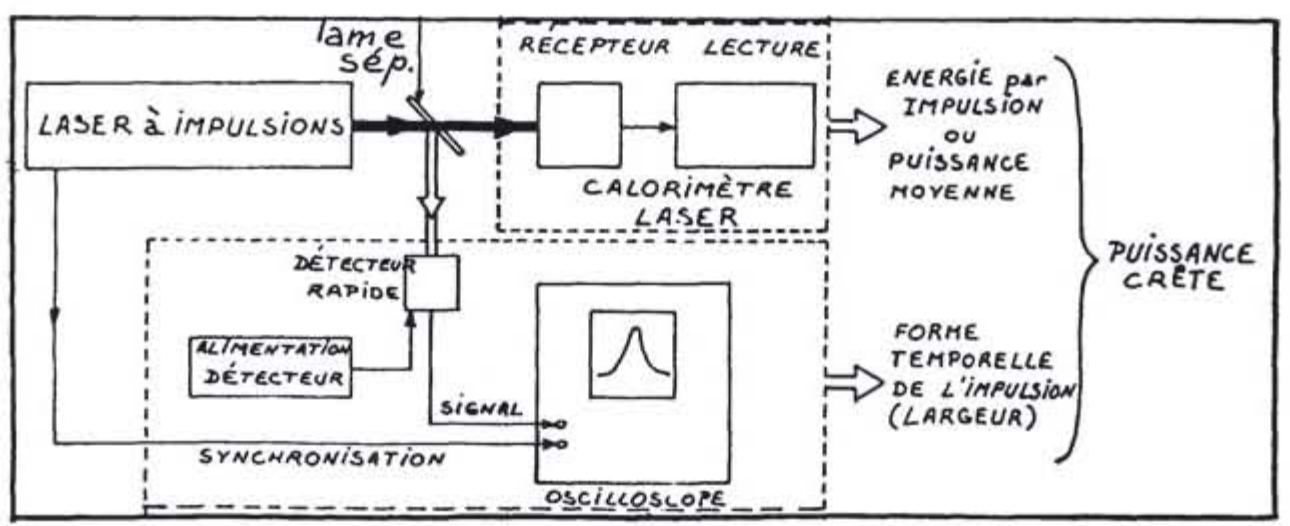

Fig III-12 : Calorimétrie pour laser Impulsionnel 
La majorité du flux émis est absorbé par un calorimètre (détecteur thermique) chargé de mesurer soit l'énergie Ei de chaque impulsion, soit le flux moyen émis s'il s'agit d'une source à fréquence de répétition FR fixe.

La mesure de ces paramètres permet par exemple de déduire le flux crête émis à chaque impulsion par le laser :

$$
\text { Fcrête }=E \mathrm{i} / \tau=\mathrm{Fm} / \tau \mathrm{FR}_{\mathrm{R}}
$$

En pratique, si le flux laser à détecter n'est pas trop important, l'absorption par le calorimètre pourra s'effectuer par exemple, sur un disque noirci à thermopile, ou à l'intérieur de formes géométriques (cônes, sphères creuses) assurant une bonne aborption du rayonnement par réflexions multiples sur leurs faces.

Si le flux laser à caractériser est puissant et peut causer des dégâts sur une surface de réception trop faible, il existe des calorimètres à absorption volumique ou partielle, le faisceau laser ne faisant que traverser le calorimètre, une fraction connue du flux étant absorbée au passage. La plupart de ces calorimètres sont autoétalonnés par une mesure de leur réponse à une excitation électrique connue dans une résistance interne.

\section{III.6. EXEMPLES DE DETECTEURS PHOTONIQUES}

\section{III.6.1. CLASSIFICATION}

Dans un détecteur photonique, l'absorption du rayonnement incident par les atomes ou molécules du matériau photosensible donne lieu à une création et récupération directe de charges libérées par le changement d'état qui en résulte. Pour que œ phénomène d'absorption puisse se produire, l'énergie des photons doit être supérieure à un seuil (appelé seuil photoélectrique, Uo) déterminé par l'énergie d'excitation des électrons du matériau.

Le changement d'état subi par un électron (appelé photoélectron) sous l'effet d'un photon incident, peut prendre les 2 formes suivantes:

- soit éjection de l'électron hors du matériau ; ce phénomène est appelé photoémission ou effet photoélectrique externe (détecteurs photoémissifs)

- soit libération, au sein du matériau, d'un électron qui assurait auparavant une liaison de valence entre atomes ou qui était retenu par attraction électrostatique à un atome étranger (impureté). Ce phénomène, dénommé effet photoélectrique interne, est exploité en détection dans les semi-conducteurs par:

- la mesure de la variation de conductivité électrique du matériau sous l'effet du rayonnement (detecteurs photoconducteurs)

- la mesure de la variation de d.d.p. aux bomes de jonctions entre zones homogènes de semi-conducteurs, due au passage des photoélectrons (détecteurs photovoltaĩques).

\section{III.6.2. DETECTEURS PHOTOEMISSIFS}

Dans ces détecteurs, les photoélectrons doivent être excités près de la surface du matériau pour pouvoir en être arrachés, puis récupérés : le matériau photoémissif est donc déposé sour forme de couche mince, sur une électrode (appelée photocathode), placée dans une ampoule à vide. Une électrode polarisée positivement (anode) attire à elle les électrons sortant de la photocathode. 
La forme la plus simple de détecteur photoémissif est la "diode photoémissive" dont le schéma général est donné sur la Figure III-13, avec la courbe caractéristique qui traduit la variation du courant en fonction de la tension appliquée entre électrodes, pour un flux incident donné.

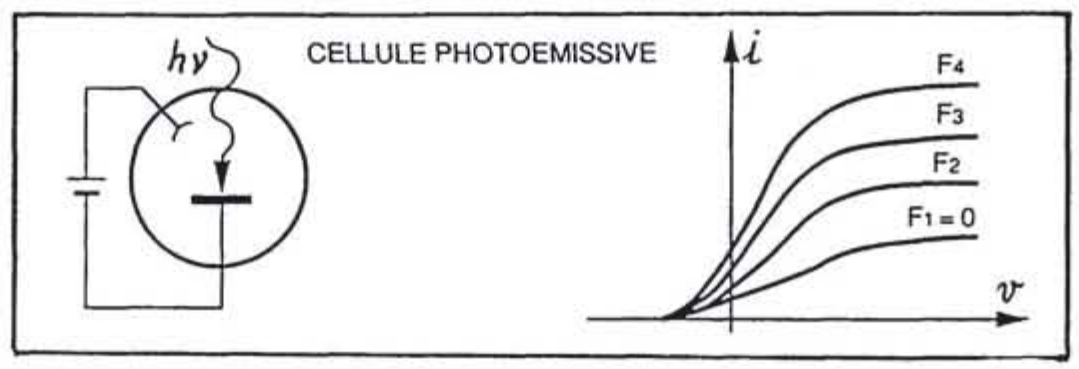

Fig III-13 : Diode photoémissive : principe et caractéristiques

D'autres configurations, plus complexes, permettent d'accroître le nombre d'électrons récupérés par l'anode (courant anodique) par lintroduction entre la cathode et l'anode d'électrodes intermédiaires convenablement polarisées pour accélérer les électrons dits primaires provenant de la cathode et créer en cascade des électrons "secondaires". On citera par exemple le cas de photomultiplicateurs oủ l'utilisation d'une dizaine de "dynodes" (électrodes intermédiaires) peut conduire à une amplification de courant de l'ordre de $10^{6}$ sur l'anode, et les intensificateurs d'images à microcanaux (électrodes en forme de tubes cylindriques fins assemblés en faisceaux) au bout desquels les électrons accélérés excitent un écran électroluminescent (Figure III-14).
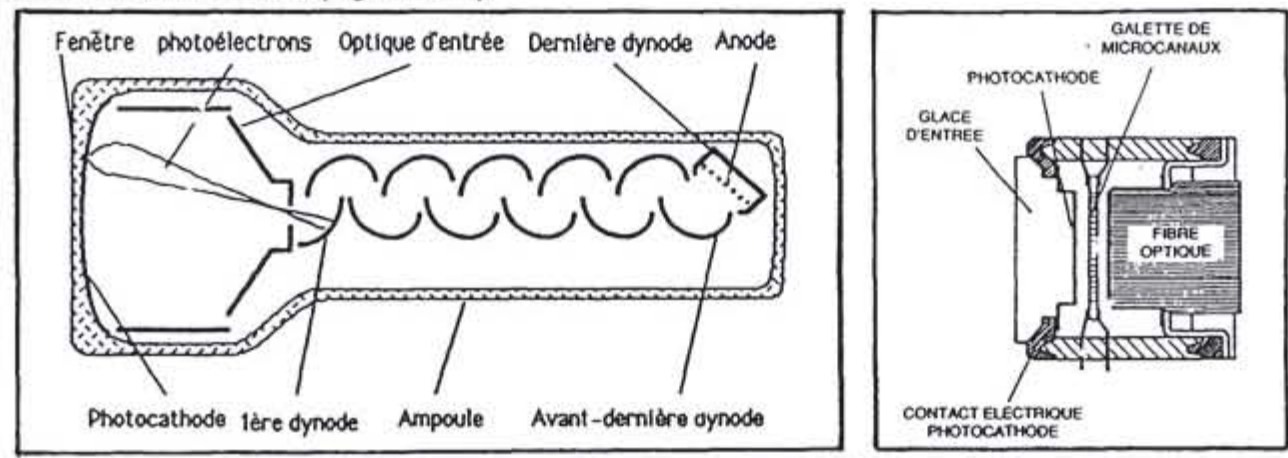

Fig III-14 : Photomultiplicateur et intensificateur à microcanaux

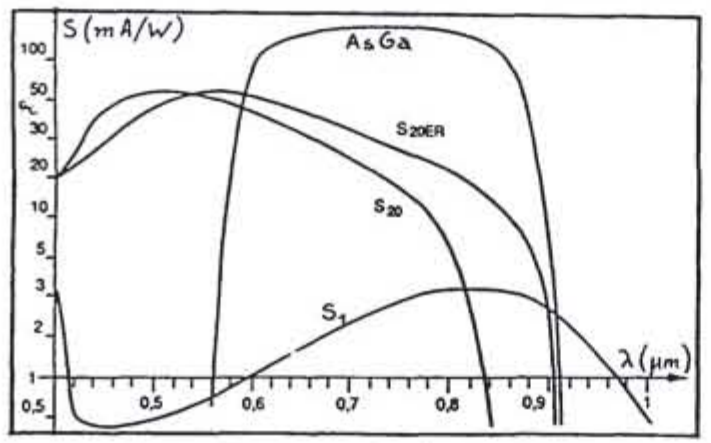

Les matériaux des photocathodes sont sensibles dans l'ultraviolet, le visible et le proche infrarouge jusqu'à $1.1 \mu \mathrm{m}$ environ). La Figure III-15 présente certains des facteurs de reponse typiques.

Flg III-15 : Facteurs de réponse spectraux de cathodes photoémissives 
III.6.3. DETECTEURS A SEMI-CONDUCTEURS

Les détecteurs à semi-conducteurs mettent en oeuvre l'effet photoélectrique interne sous 2 modes : photoconducteur et photovoltaïque.

DETECTEURS PHOTOCONDUCTEURS : L'absorption d'un rayonnement d'énergie photonique suffisamment élevée, par les électrons d'un matériau semi-conducteur homogène, y provoque une augmentation de la densité des charges libres, donc de sa conductivité électrique. Un tel semi-conducteur se comporte en détecteur (photoconducteur) de la lumière par l'augmentation de sa conductance sous l'effet du rayonnement. Comme dans le cas des bolomètres, une polarisation extérieure est nécessaire pour exploiter cette variation de résistance. La Figure III-16 représente le montage et la caractéristique typiques d'un détecteur en mode photoconducteur.

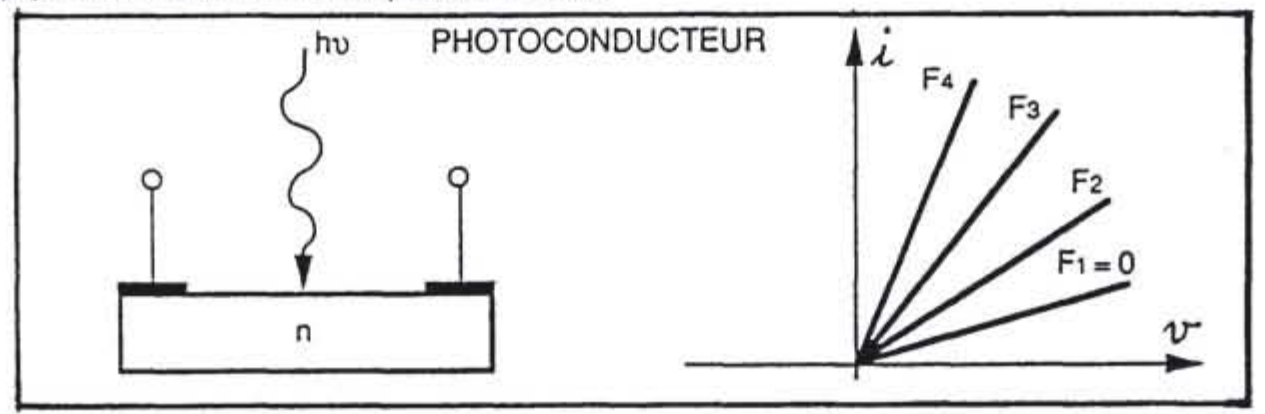

Fig III-16 : Détecteur photoconducteur: caractéristique

Les semi-conducteurs envisageables pour la réalisation de détecteurs photoconducteurs sont :

- soit intrinsèques : la transition des photoélectrons s'effectue de la bande de valence à la bande de conduction

- soit extrinsèques

- de type $\mathrm{N}$ (conduction par électrons) : la transition s'effectue depuis les niveaux d'impureté (situés dans la bande interdite du semi-conducteur) à la bande de conduction

- de type $\mathrm{P}$ (conduction par "trous") : la transition s'effectue de la bande de valence à un niveau d'impureté.

Les schémas de la Figure III-17 résument les phénomènes engagés dans l'excitation des électrons de ces 3 types de semi- conducteurs:

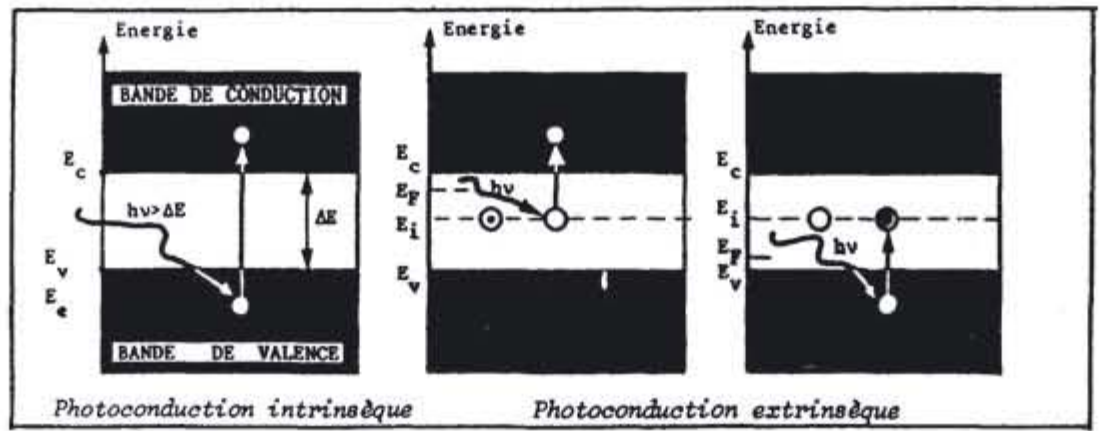

Fig III-17: Création de charges dans les semi-conducteurs intrinseqques ou extrinseqques ( $N$ et $P$ )

Parmi les semi-conducteurs "intrinsèques", on citera $\mathrm{PbS}, \mathrm{PbSe}, \mathrm{PbTe}, \mathrm{InSb}, \mathrm{AsGa}$, InAs, HgCoTe et parmi les extrinsèques, Ge et Si dopés par $\mathrm{Au}, \mathrm{Hg}$, $\mathrm{Cu}$. 
DETECTEURS PHOTOVOLTAlOUES : L'utilisation d'un semi-conducteur dans le mode photovoltaĩque suppose par définition la présence de une ou plusieurs jonctions entre régions homogènes (dopées $\mathrm{N}$ et $\mathrm{P}$ ) de ce semi-conducteur. Si le composant ne comporte qu'une jonction, il s'agit d'une photodiode ; s'il en comporte 2. c'est un phototransistor qui intègre à la fois les fonctions de détection et de préamplification de courant (typiquement de 50 à 200) par effet transistor ; s'il y a 3 jonctions, on a affaire à un photothyristor.

Une jonction éclairée par un flux lumineux de longueur d'onde $\lambda<\lambda c$, absorbe une fraction des photons incidents en créant des paires électron-trou, aussi bien dans les régions homogènes qu'à l'interface (zone de charge d'espace). C'est là que le phénomène est le plus important car chaque porteur créé dans cette zone est soumis au champ local, les électrons étant accélérés vers la zone $\mathrm{N}$ et les trous vers la zone $\mathrm{P}$. Ainsi séparés, ils ne peuvent plus se recombiner et contribuent à accroître le courant d'une valeur proportionnelle au flux incident. La Figure III-18 montre la caractéristique (i,v) d'une photodiode qui fait apparaître 2 régions de fonctionnement :

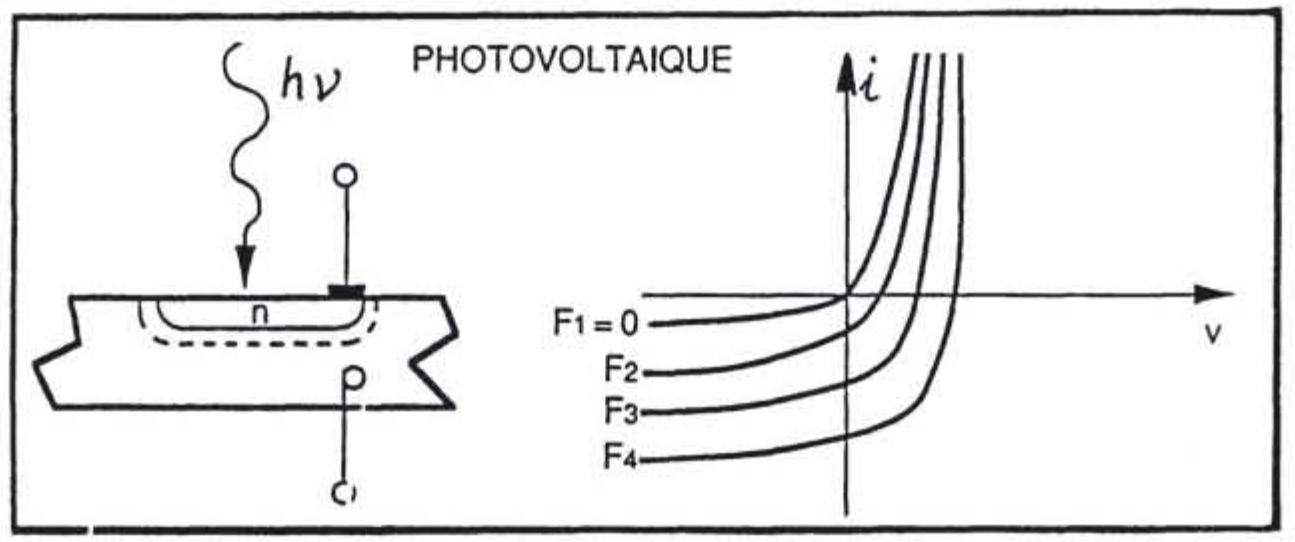

Fig III-18 : Courbes caractéristiques d'une photodiode

En l'absence de polarisation externe (ou sous faible polarisation en direct), la diode se comporte, sous l'effet du flux, comme un générateur électrique : cette utilisation en "photopile" est mise à profit dans la réalisation de capteurs ou panneaux solaires de grandes dimensions destinés à fournir de l'électricité à bord de satellites ou dans les pays à fort ensoleillement.

En polarisation inverse (ou contre polarisation) le détecteur fonctionne en régime de "photodiode* proprement dit. La configuration précise de la diode (largeur, position de la zone de charge d'espace, tension appliquée) est à définir en fonction de paramètres tels que rapidité de réponse, optimisation de la réponse spectrale dans tel domaine de longueur d'onde. On mentionnera simplement ici quelques types parmi les plus courants tels que photodiodes P, N, photodiodes PIN, photodiodes Schotthy, photodiodes à avalanche, ....

Le mode de détection photovoltaique ne peut mettre en oeuvre que des semi-conducteurs intrinséques (création de paires électron-tron), ce quil limite le choix des matériaux utilisables par rapport aux possibilités du mode photoconducteur. Par contre, les détecteurs en mode photovoltaĩque présentent l'avantage d'une plus grande rapidité (bandes passantes de plusieurs gigahertz) et d'un bruit plus réduit (abser.ce de recombinaison) qu'en mode photoconducteur. 


\section{III.6.4. DETECTEURS POUR L'IMAGERIE}

Si ron ne tient pas compte des détecteurs pyroélectriques, l'imagerie repose essentiellement sur l'utilisation de détecteurs quantiques (photoémissifs et semi-conducteurs). Les géométries mises en oeuvre sont très différenciées, depuis l'association de détecteurs monoéléments à des systèmes de balayage optomécaniques (caméras thermiques de mesures) jusqu'aux matrices dites "dans le plan focal", dont le nombre d'éléments est égal à celui des points dans l'image, en passant par les barrettes linéaires ou matrices à faible nombre d'éléments.

Dans le domaine du visible et du proche infrarouge, les premiers analyseurs d'images ont été constitués de tubes à vide dont la face d'entrée était recouverte soit d'une couche photoconductrice $\left(\mathrm{Sb}_{2} \mathrm{~S}_{3}\right)$. sensible dans le visible, soit d'une mosaĩque de photodiodes au silicium (sensible jusqu'à 1 um environ)(Figure III-15)

Ces tubes sont remplacés actuellement par les détecteurs à transfert de charges (DTC, ou en anglais, CCD : Charge Coupled Devices). Ces demiers sont des mosaïques de photodiodes au silicium associées à des structures à effet de champ (capacités MOS, ou Metal-Oxide Semiconductor), qui assurent le transfert séquentiel des photoélectrons (créés par les diodes) vers le circuit d'exploitation (Figure III-19).

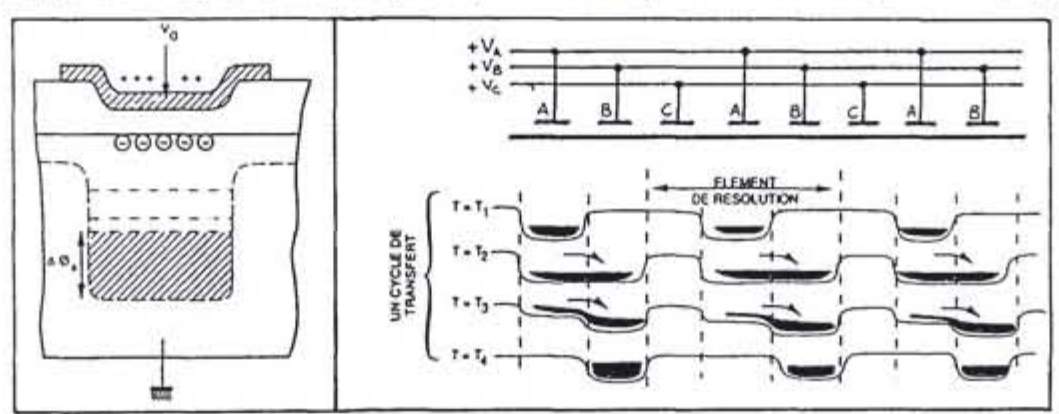

Fig III-19 : Détecteur CCD : capacité MOS

Ce type de détecteurs matriciels à l'état solide possédant un grand nombre de points voit actuellement son utilisation limitée au visible et au proche IR, mais présente également un très grand intérêt dans l'infrarouge : il permettrait de supprimer les systèmes de balayage optomécanique présents dans les caméras infrarouges actuelles (caméras thermiques ou FLIRs : Fonward Looking Infrared Systems) et qui sont la source de surcoûts importants. De nombreux travaux sont actuellement en cours pour la réalisation de telles matrices, soit dans la bande $3 / 5 \mu \mathrm{m}$ (matrices de diodes Shottky Pt-Si, matrices InSb) soit dans la bande $8 / 12 \mu \mathrm{m}$ (matrices $\mathrm{HgCdTT}$, ou MCT).

III.6.5. REFROIDISSEMENT DES DETECTEURS PHOTONIQUES

II a été noté plus haut, dans la description générale des détecteurs ph stoniques, que la création de photoélectrons provient de labsorption des photons du rayonnement à détecter. II existe malheureusement d'autres causes que le rayonnement recherché dans l'excitation des électrons du détecteur, telles que l'agitation thermique des atomes et molécules du matériau photosensible et/ou l'éclairement du détecteur par le fond environnant. Ces sources de signaux parasites peuvent masquer plus ou moins le phénomène recherché et doivent être combattues de façon appropriée.

On s'attachera généralement à réduire lagitation thermique du détecteur nt le rayonnement ambiant qu'il reçoit en refroidissant le détecteur et en limitant son champ de vision à la valeur utile au moyen d'une 
enceinte à diaphragme elle-même refroidie. Les méthodes de refroidissement à mettre en place ainsi que les températures à atteindre sont essentiellement fonction de la bande spectrale de fonctionnement du système et de la sensibilité requise pour le détecteur. Le tableau ci-dessous donne la correspondance généralement acceptée entre niveau de température, méthode de refroidissement et domaine spectral de fonctionnement.

Tableau III-4 : Températures et domaines de fonctionnement de détecteurs

\begin{tabular}{|c|c|c|c|c|c|}
\hline \multirow[t]{2}{*}{ Température } & \multirow[t]{2}{*}{ Méthode } & \multicolumn{4}{|c|}{ Bande spectrale } \\
\hline & & Visible & $1 / 3 \mu \mathrm{m}$ & $3 / 5 \mu \mathrm{m}$ & $>8 \mu \mathrm{m}$ \\
\hline ambiante & & tous & $\begin{array}{l}\mathrm{Ge} \\
\mathrm{PbS} \\
\text { InGaAs }\end{array}$ & $\begin{array}{l}\text { PbSe } \\
\text { HgCdTe }\end{array}$ & Non \\
\hline $\begin{array}{l}250 \mathrm{~K} \\
200 \mathrm{~K}\end{array}$ & $\begin{array}{c}\text { Effet Peltier } \\
1 / 2 \text { étages } \\
4 \text { étages }\end{array}$ & $\begin{array}{c}\mathrm{CCD} \\
\text { (Astronomie) }\end{array}$ & $\mathrm{PbS}$ & $\begin{array}{c}\mathrm{PbS} \\
\mathrm{PbSe} \\
\mathrm{HgCdTe}\end{array}$ & Non \\
\hline $77 \mathrm{~K}$ & $\begin{array}{c}\text { Détente Joule } \\
\text { Thomson (air, azote) } \\
\text { Dewar azote liquide } \\
\text { Machine cryogénique }\end{array}$ & & & $\begin{array}{l}\text { PbSe } \\
\text { HgCdTe } \\
\text { InSb } \\
\text { PISi }\end{array}$ & $\mathrm{HgCdTe}$ \\
\hline $30 \mathrm{~K}$ & \begin{tabular}{|c|}
$\begin{array}{c}\text { Dewar hydrogène } \\
\text { liquide }\end{array}$ \\
\end{tabular} & & & & $\mathrm{Ge} \mathrm{Hg}$ \\
\hline $4 \mathrm{~K}$ & Dewar hélium liquide & & & & $\mathrm{Ge} \mathrm{Cu}$ \\
\hline
\end{tabular}

Les principales méthodes de mise en froid de détecteurs sont : l'effet Peltier, la détente Joule Thomson, les machines cryogéniques, l'utilisation de gaz liquéfiés.

L'effet Peltier (ou thermoélectrique) consiste dans le refroidissement (ou échauffement) provoqué par le passage d'un courant électrique dans la jonction entre deux métaux différents. "I présente l'avantage de n'exiger qu'une source d'énergie électrique, conduit à des dispositifs fiables et compacts (le détecteur repose sur la jonction froide constituée d'une plaque de cuivre en contact avec du tellurure de Bismuth) et ne pose pas de problèmes dus aux vibrations. Par contre, les températures minimales accessibles qui demeurent élevées, dépendent du nombre d'étages et de la température ambiante (par exemple, $195 \mathrm{~K}$, avec 6 étages). D'autre part, il faut veiller soigneusement à la qualité du contact thermique entre le détecteur et la "plaque froide" (Figure III- 20a)

Le refroidissement par détente Joule Thomson exploite l'abaissement de la température par la détente d'un gaz à haute pression : le gaz sous pression (par exemple de l'azote entre 200 et 400 bars) se détend dans une sonde qui est introduite à l'intérieur du Dewar contenant le détecteur. La sonde est constituée d'un manchon thermiquement isolant sur lequel est bobiné un tube métallique fin, par l'extrémité duquel s'échappe le gaz décompressé, donc refroidi. Au bout de quelques minutes, le tube est assez froid pour qu une gouttelette d'azote se forme au contact du détecteur (Figure III-20b). Le refroidissement par machine cryogénique s'opère mécaniquement par détente d'un gaz comprimé suivant plusieurs possibilités de cycles thermodynamiques. Parmi ces demiers, on citera le cycle de Sterling (phases de compression et de détente isothermes, et régénération à volume constant) et le cycle de Vuilleumier (apport de chaleur par chauffage électrique).

Enfin, le refroidissement par gaz liquéfié consiste à placer le détecteur en contact avec le gaz liquéfié (tel que l'azote) contenu dans un réservoir, généralement un vase Dewar comprenant une double enveloppe en verre ou en métal isolée par un vide poussé (cryostat). Le gaz liquéfié s'évapore lentement, ce qui assure au détecteur une autonomie de plusieurs heures. 


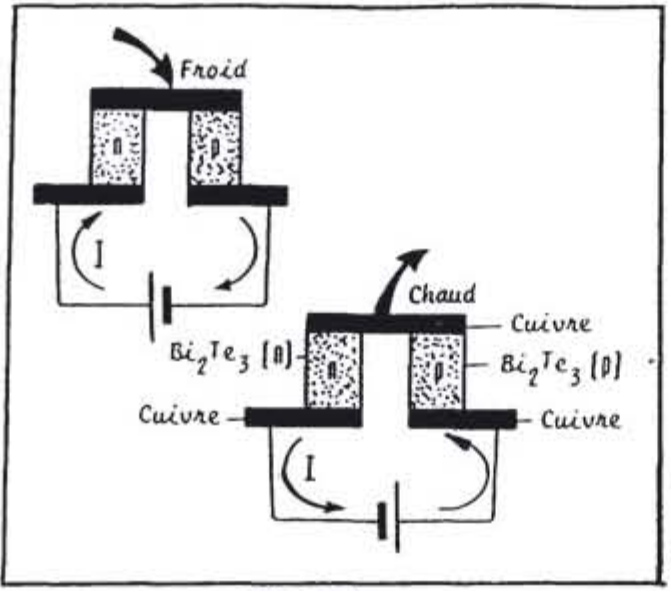

a- Effet Peltier

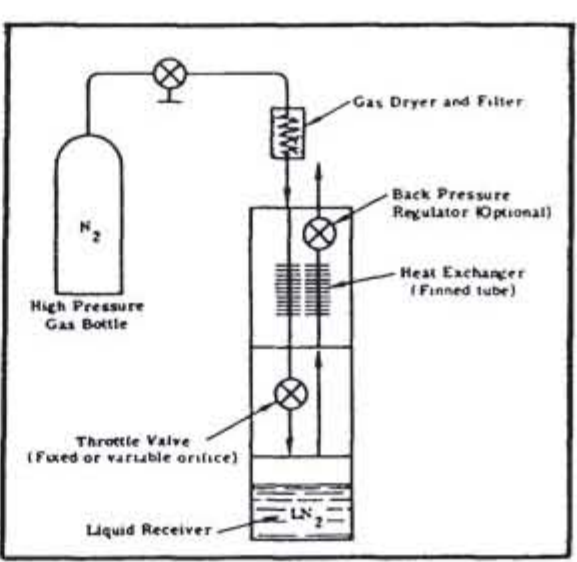

b-Sonde Joule Thomson

Fig III-20 : Refroidissement de détecteur 


\section{CHAPITRE IV LA DETECTION OPTIQUE}

Après les chapitres (et les cours) précédents destinés à l'analyse des sous-ensembles constitutifs de systèmes optiques ou optroniques, ce chapitre a pour objet de présenter les procédures les plus couramment utilisées pour évaluer les performances en détection de ces systèmes.

Pour cela, on rappelle tout d'abord (paragraphe IV.1) le problème général de la détection de signaux, que I'on trouve non seulement en optique, mais aussi dans beaucoup d'autres domaines tels que le radar, les télécommunications et les transmissions, la mesure automatique, la reconnaissance de forme, etc... Le paragraphe IV.2 illustre qualitativement le problème de la détection optique par l'exemple classique de l'observation d'une scène.

Traditionnellement, on distingue deux classes de systèmes optiques de détection : la première (traitée au paragraphe IV.3) comprend les systèmes dits "à détection automatique", c'est-à-dire sans aucune intervention humaine dans le fonctionnement de l'appareil. Dans la seconde classe, un opérateur humain intervient, soit pour une observation visuelle de la scène au travers du système (systèmes dits "visuels" tels que jumelles, périscopes, ... traités au paragraphe IV.4), soit pour l'observation d'un écran (systèmes dits "à visualisation" tels que caméras de télévision ou infrarouge, paragraphe IV.5).

\section{IV.1. POSITION GENERALE DU PROBLEME DE LA DETECTION OPTIQUE}

Les performances en détection d'un système optique se définissent en premier lieu à partir des objectifs et de la mission assignés à ce système. Par exemple, la performance d'un système optique de télécommunications pourra s'exprimer au moyen de son taux d'erreur par bit. Dans le cas d'un système optique chargé de la surveillance et de la défense d'une zone, la performance pourra se traduire par sa portée en détection, c'est-à-dire la limite en distance au-delà de laquelle la surveillance n'est plus assurée avec les qualités requises. Pour un système d'imagerie infrarouge, on exprimera sa performance par la différence de température minimale qu'il permet de détecter, etc...

Dans chaque cas, on prendra soin d'analyser finement les caractéristiques spatiales, spectrales et temporelles du signal à détecter pour $y$ adapter au mieux le système optique que l'on cherche à concevoir, mais cette approche ne suffit pas pour déduire la performance du système. En effet, d'autres paramètres interviennent, tels que les pertes dues au milieu de propagation, qui peuvent dégrader le signal recherché, ou le masquer parmi d'autres sources, parasites, de rayonnements.

De façon générale, le signal délivré par un système optique est un signal plus ou moins bruité" et le problème général de la détection est de décider de la présence ou de l'absence de l'objet recherché à l'issue de l'examen de ce signal.

Pour juger de la performance en détection d'un système optique, on dresse "la table de vérité" suivante. basée sur la réponse du système (seul ou avec un observateur humain) en fonction de la réalité (absence ou présence de robjet ou du signal optique recherché) :

\begin{tabular}{|c|c|c|}
\hline $\begin{array}{c}\text { Situation réelle } \rightarrow \\
\text { Réponse système } \downarrow\end{array}$ & Présence & Absence \\
\hline Présence & Détection & Fausse alarme \\
\hline Absence & Non détection & Veille \\
\hline
\end{tabular}


Le comportement attendu du système est de détecter l'objet quand œe dernier est réellement présent ("détection") et de ne pas réagir lorsque l'objet est absent (état de veille). Les défauts de fonctionnement apparaissent lorsque le système ne permet pas de percevoir l'objet quand il est présent ("non détection") ou lorsqu'il signale sa présence alors que l'objet n'est pas là (cas de "fausse alarme").

En moyennant les résultats sur un nombre suffisamment grand de mesures, on dégage ainsi les 2 paramètres les plus usités dans l'évaluation de performance en détection d'un système :

- La probabilité de détection $\left(\mathrm{Pd}_{\mathrm{d}}\right)$ : c'est la probabilité pour que le système détecte un objet réellement présent

- La probabilité de fausse alarme (PFA): c'est la probabilité pour que le système détecte la présence de l'objet alors que ce demier est absent.

On conçoit que la performance du système optique sera d'autant meilleure (Pd proche de $100 \%$ et PFA aussi faible que possible) que le signal optique ou l'objet observé se distingue bien du fond et/ou du bruit propre du système.

La suite de ce chapitre montrera que le paramètre important dans la performance d'un système optique est la notion de "rapport signal à bruit" présentée dans les paragraphes IV.3 et IV.4. II est nécessaire que le signal utile soit aussi fort que possible vis-à-vis du bruit car s'il est nul ou très faible, il ne pourra pas être récupéré, ou difficilement, par le traitement de signal même le plus sophistiqué (comme disent les Américains : "Garbage in, Garbage out").

\section{IV.2. EXEMPLE : OBSERVATION D'UNE SCENE}

Pour illustrer les idées générales ci-dessus, en particulier sur la notion de signal optique, considérons de façon plus ou moins qualitative le cas, courant en optique, de l'imagerie ou de l'observation d'une scène. qui peut être représentatif d'une des situations ci-dessous :

- Observation d'un paysage en direct

- Observation avec des jumelles

- Imagerie télévision et observation sur moniteur

- Système d'observation de la terre depuis un satellite (SPOT)...

Dans chaque cas, la scène est caractérisée par un ensemble d'objets, parmi lesquels celui (ou ceux) que l'on cherche à détecter (à reconnaitre etc...), les fonds sur lesquels apparaissent ces objets, les sources qui éclairent la scène (soleil, ciel, ...), le ou les milieux de propagation, etc...

Le calcul du rayonnement des sources et des objets peut se faire au niveau de chaque émetteur grâce aux résultats du chapitre II, par exemple au moyen de la décomposition, faite au paragraphe II.1.4, en émission propre et réflexion de réclairement ambiant. A partir de là, il convient de tenir compte des propriétés du milieu de propagation, tel que latmosphère qui, sur des distances d'observation importantes, joue plusieurs rôles perturbateurs (voir paragraphe 1.4.4) sur la performance du système optique :

- Atténuation du rayonnement utile recherché, par absorption et diffusion des photons par les atomes, molécules et particules (aérosols) en suspension.

- Emission de rayonnements parasites par diffusion de la lumière ambiante et émission propre par ces mêmes molécules et particules

- Dans certains cas, déformation des rayons vis-à- vis de la propagation rectiligne de la lumière, à cause des inhomogénéités d'indices rencontrées (influence de la turbulence, citée ici pour mémoire). 
Ainsi, tout système optique destiné à observer une scène au travers de l'atmosphère perçoit le long de chaque direction située à lintérieur de son champ de vision, une luminance apparente de scène (c'est-à-dire mesurée au niveau de sa pupille), qui est généralement la superposition de plusieurs termes et en particulier (Fig IV-1):

- La luminance apparente Lu du rayonnement à détecter (rayonnement "utile" ou de signal)

- La luminance apparente $L_{p}$ due à l'émission propre de l'atmosphère (conséquence de la loi de Kirchhoff, le facteur d'absorption de l'atmosphère n'étant pas nul).

- La luminance apparente Lo du rayonnement solaire et ambiant diffusé par l'atmosphère.

La luminance apparente "vue" par le système dans chaque direction de l'espace peut alors s'exprimer, de façon générale, par la relation suivante:

$$
L_{\text {app }}=L_{u}+L_{p}+L_{d}
$$

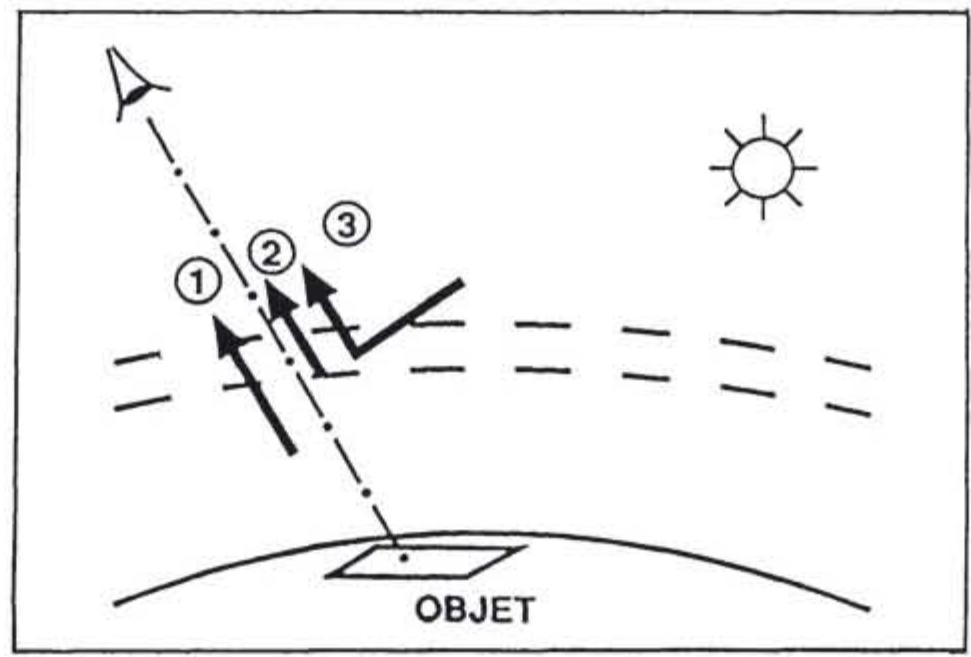

Fig IV-1 : Luminances apparentes utile et parasite pour un système d'observation

La capacité de tout système optique d'observation à détecter la présence de l'objet (signal) ou à le discriminer vis-à-vis du fond environnant, repose essentiellement sur la différence entre la luminance apparente de l'objet et celle du fond.

Cela peut se vérifier, par exemple, dans l'observation visuelle d'un paysage : on en distingue plus ou moins facilement les différentes zones, suivant les conditions atmospheriques. Lorsque ces dernières évoluent depuis le beau temps jusqu'au brouillard, en passant par les diverses étapes de la brume, la part de luminance utile de l'objet dans sa luminance apparente s'atténue de plus en plus, par rapport à celle de la diffusion, de telle sorte que les luminances apparentes d'objet et de fond deviennent si proches l'une de l'autre que l'objet n'est plus discernable du fond.

Cet exemple simple rappelle que, pour qu'un objet ou un signal soit détecté par un système optique, il faut que leur présence entraine une réponse ou une variation de réponse de $œ$ demier, que l'on traduit en notion de contraste cians le cas des systèmes visuels ou à imagerie.

Bien que très importante, œette notion de contraste n'est cependant pas suffisante pour définir les limites en détection d'un système, car il faut prendre en compte les fluctuations de signal dues au bruit du détecteur. 
Les procédures les plus généralement utilisées pour évaluer la qualité en détection de systèmes optiques se fondent donc sur 2 paramètres : le contraste et le rapport signal à bruit, qui sont définis dans la suite de $œ$ chapitre. L'utilisation pratique de ces paramètres y est illustrée pour les 3 types de systèmes optiques : automatiques, visuels et à visualisation.

\section{IV.3. DETECTION AUTOMATIQUE}

\section{IV.3.1. SYSTEMMES A "SEUIL"}

Les systèmes optiques à détection automatique traitent eux-mêmes le signal électrique délivré par leur détecteur pour en déduire, sans intervention humaine, la présence éventuelle, dans leur champ de vision, du rayonnement, message ou objet recherché. Ces appareils remplissent par exemple des fonctions de surveillance, détection d'intrusion, transmission/réception de messages, télécommunications, télémétrie. etc...

La prise de décision ("absence/présence") par de tels dispositifs s'effectue à partir de divers critères, dont le plus utilisé repose sur la comparaison entre le signal électrique délivré par le capteur (généralement une tension, v) et une certaine valeur de référence (qui peut être fixe ou variable dans le temps), appelée "seuil". La décision prise par le système, dictée par cette comparaison, peut être telle que :

$$
\begin{aligned}
& v>S \rightarrow \text { présence de signal utile } \\
& v \leq S \rightarrow \text { absence de signal utile }
\end{aligned}
$$

L'une des difficultés dans la conception de tels systèmes réside dans la détermination de la valeur du seuil : en effet, il a été vu plus haut (paragraphe III.3.2) que la tension électrique aux bomes de la résistance de charge du détecteur présente, même en absence de signal utile, des fluctuations de niveau dues aux différentes sources de bruit du système. Si le seuil est placé à une valeur trop basse, près des pointes du bruit, le système sera très sensible aux fausses alarmes. Par ccntre, si la valeur du seuil est trop élevée, le système détectera très rarement la présence du signal utile (d'où une probabilité de détection faible) (Fig IV-2).

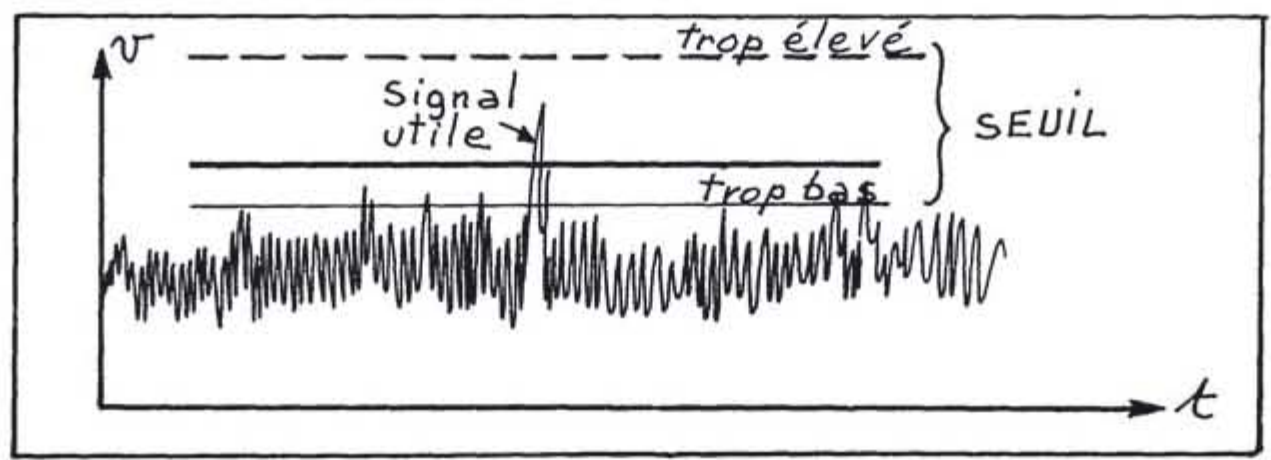

Flg IV-2 : Positlonnement du seull d'un système automatique

Le problème est d'adapter au mieux la valeur du seuil aux spécifications du système en détection, généralement données sous la forme dune probabilité de détection $\mathrm{Pd}$ et d'une probabilité de fausse alarme PFA. Pour cla, on caractérisera la tension 'je sortie (sur laquelle on va définir le seuil) par sa densité de probabilité qui est la bi $p(v)$, telle que la probabilité pour que la tension $V$ soit comprise entre $v$ et $v+d v$ soit égale à :

$$
P(v<V<v+d v)=p(v) d v
$$


La densité de probabilité de la tension en sortie du système dépend essentiellement :

- Du mode de détection utilisé (directe ou hétérodyne)

- De la présence/absence de signal utile

- Des paramètres du signal tels que sa "force" et ses fluctuations d'un échantillonnage à l'autre.

La figure IV-3 illustre qualitativement rallure de ces courbes pour 3 cas, en présence de bruit seul (A), en présence de signal utile faible (B), et en présence de signal utile "fort" (C).

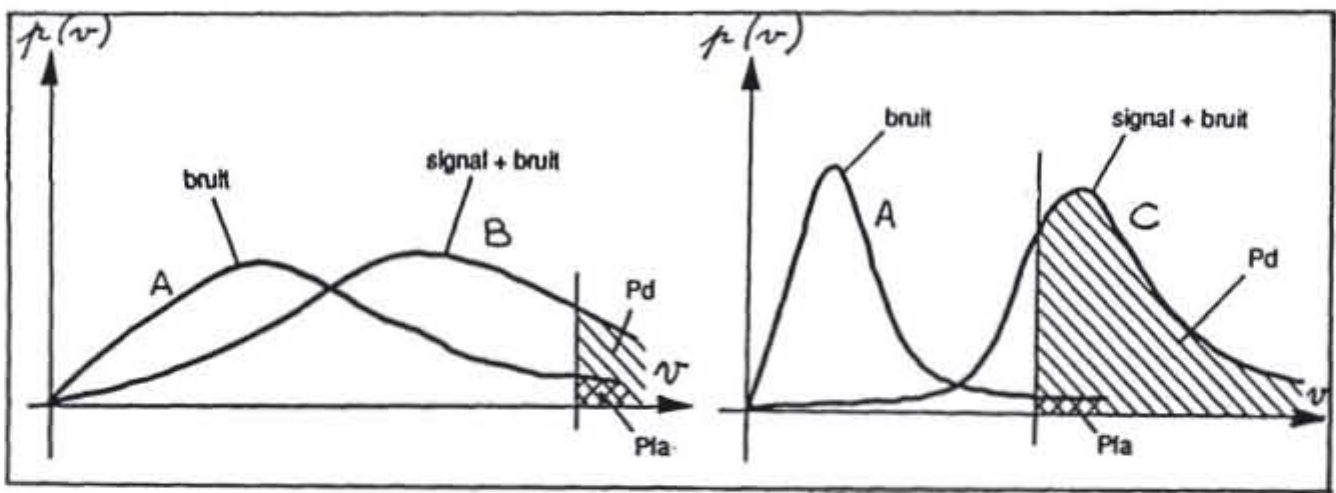

Figure IV-3: Densités de probabilité de tension de sortie

La probabilité de détection et la probabilité de fausse alarme du système qui sont les 2 paramètres de base dans l'évaluation de ses performances, s'expriment alors de la façon suivante, en fonction des 2 lois de densité de probabilité de la tension (en présence et en absence de signal utile) et de la valeur $\mathrm{S}$ du seuil :

$$
\begin{aligned}
& \mathrm{P}_{\mathrm{d}}=\text { probabilité pour que } v_{S+B}>\mathrm{S}=\int_{S}^{\infty} p\left(V_{S+B}\right) d v \\
& \text { et } \mathrm{PFA}_{\mathrm{FA}}=\text { probabilite pour que } \mathrm{VB}>\mathrm{S}=\int_{S}^{\infty} p\left(v_{B}\right) d v
\end{aligned}
$$

\section{IV.3.2. DÉtECTION AUTOMATIQUE ET RAPPORT SIGNAL A BRUIT}

II ressort du paragraphe précédent que la performance d'un système de détection ne dépenid pas de la valeur dans labsolu du signal électrique utile qu'il delivre mais plutôt de ses valeurs en signal et en bruit. exprimées par l'intermédiaire de leurs densités de probabilités respectives.

II est clair que la courbe de densité de probabilité de la tension de signal utile se differencie d'autant plus de celle du bruit seul et se décale d'autant plus vers les fortes valeurs de tensions (c'est-à-dire vers la droite sur Paxe des abscisses) que le signal (attendu ou mesure) est important : pour les signaux "forts", on pourra établir le seuil à une valeur $\mathrm{S}$ élevée par rapport au bruit pour réduire au minimum la probabilité de fausse alarme sans affecter de façon significative la probabilité de détection. Par contre, pour les signaux "fables", on constate qu'une élévation du niveau de seuil réduit rapidement la probabilité de détection. 
Sans entrer dans le détail des lois de densités de probabilité, on peut dire que pour un mode de détection donné, leur expression mathématique dépend essentiellement de la valeur moyenne (et des fluctuations d'une mesure à l'autre) d'un paramètre, appelé "rapport signal à bruit S/B" tel que :

$$
\mathrm{S} / \mathrm{B}=\mathrm{FS} / \mathrm{NEP}
$$

oủ Fs est le flux de signal optique reçu par le détecteur et NEP le flux équivalent au bruit, ces 2 quantitès étant mesurées au même point du circuit électronique (en général aux bornes de la résistance de charge du détecteur) et dans la même bande passante électronique (en général celle du traitement adaptė au signal).

D'après les expressions du NEP données plus haut (paragraphe III.3.3 et III.4) en modes de détection directe et hétérodyne, le rapport S/B prendra, suivant les cas, les valeurs du tableau IV.1 ci-dessous :

Tableau IV.1 - Valeurs du rapport S/B

\begin{tabular}{|c|c|c|}
\hline & \multicolumn{2}{|c|}{ MODE DE DÉTECTION } \\
\hline & DIRECTE & HÉTÉRODYNE \\
\hline Rapport S/B & $D^{*} \frac{F_{S}}{\sqrt{A_{0}} \sqrt{B}}$ & $\eta_{\frac{F}{h v B}}$ \\
\hline Remarque 1 & $\begin{aligned} \mathrm{S} / \mathrm{B} & =\mathrm{iS} /\left(\mathrm{ib}^{2}\right)^{1 / 2} \\
& =\mathrm{vS}\left(\mathrm{V}_{\mathrm{b}}{ }^{2}\right)^{1 / 2}\end{aligned}$ & $\mathrm{~S} / \mathrm{B}=\mathrm{i}^{2} \mathrm{HET} / \mathrm{ib}{ }^{2}$ \\
\hline Remarque 2 & $\begin{array}{l}\text { Rapport en courant } \\
\text { ou tension } \\
\text { "Rapport S/B vidéo" }\end{array}$ & $\begin{array}{l}\text { Rapport en } \\
\text { puissances } \\
\text { électriques }\end{array}$ \\
\hline Unités & Linéaires & en dB \\
\hline
\end{tabular}

On notera bien que le rapport signal à bruit d'un système optique (rapport de 2 flux) se traduit au niveau électrique par un rapport S/B dit "vidéo" (rapport de tensions ou de courants électriques) en détection direrte et par un rapport entre puissances électriques en détection hétérodyne

\section{IV.3.3. DÉTERMINATION DU SEUIL}

Les calculs exacts des probabilités de détection et de fausse alarme de systèmes électromagnétiques ont été effectués, initialement pour des domaines tels que le radar et les télécommunications, et les résultats regroupés en un ensemble de graphes ou abaques, qui définissent la variation de la probabilité de détection (en ordonnée) en fonction du rapport signal à bruit (en abscisse) pour une probabilité de fausse alarme donnée. La valeur du seuil recherché correspond au rapport S/B minimum qui assure la probabilité de détection désirée.

La procédure d'utilisation de ces abaques pour la recherche de seuil s'appuie typiquement sur les étapes suivantes (illustrées par la figure IV-4):

1) Pointer sur l'axe des ordonnées la valeur de probabilité de détection assignée au système (exemple : $90 \%)$

2) Rechercher sur la courbe paramétrique correspondant à la probabilité de fausse alarme imposée, le point de même probabilité de détection : c'est lintersection de la droite horizontale $\left(P_{d}=c^{t \theta}=0,9\right)$ avec courbe paramétrique $P_{F A}=$ cte (exemple: $P_{F A}=10^{-12}$ )

Si le système est spécifié par son taux de fausses alarmes TFA (nombre maximal de fausses alarmes f unité de temps) et la fréquence de répétition des mesures $F_{R}$, la probabilité de fausse alarme est alors

$$
P_{F A}=T_{F A} / F_{R}
$$

3) L'abscisse de ce point est la valeur du seuil (en rapport $S / B$ ) à imposer à chaque mesure. 


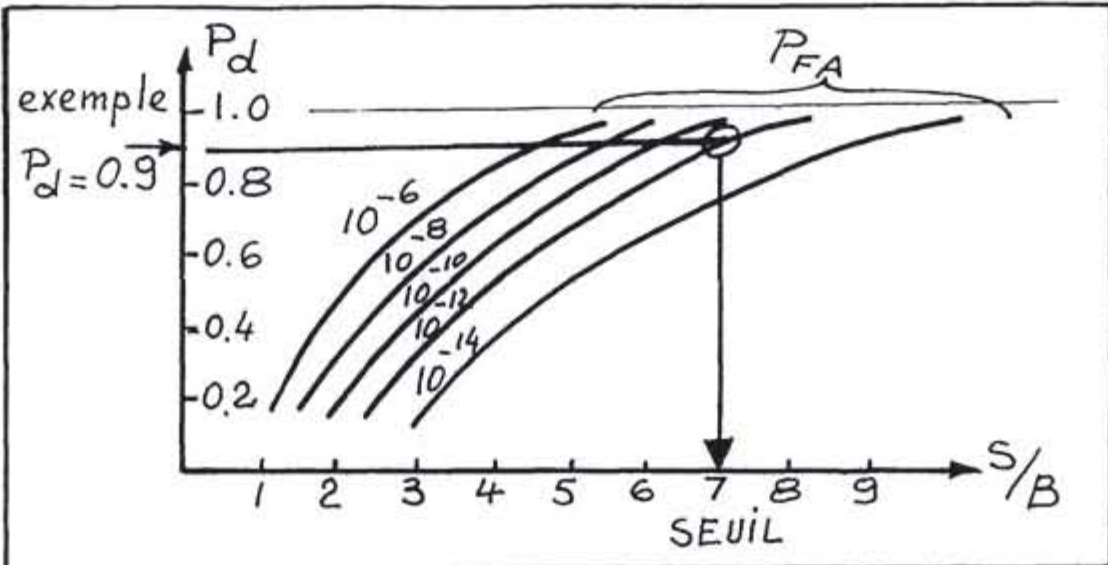

Fig IV-4 : Procédure pour la détermination du seuil d'un système de détection

En pratique, il existe un assez grand nombre d'abaques et le choix approprié dépend essentiellement des paramètres suivants :

- le mode de détection utilisé (directe ou hétérodyne)

- le caractère fluctuant ou non du signal, d'une mesure à une autre

- la statistique du bruit

La notion de fluctuation de signal, très importante par exemple dans les systèmes radar ("fluctuations de cible") est à prendre en compte dans les systèmes optiques "actifs" (possédant leur propre illuminateur). en particulier dans le cas d'éclairage cohérent (sources laser monomodes). Dans ce cas, le phénomène de "speckle" ou "granularité laser" peut entrainer d'une mesure à une autre, une variation importante de l'éclairement moyen du senseur, qui doit être prise en compte dans la statistique du signal.

On donne ci-dessous trois types d'abaques applicables aux systèmes optiques et correspondant aux 3 cas suivants : Fig. IV.5 en détection directe; Fig IV. 6 et IV.7 en détection hétérodyne (signaux fluctuant et stationnaire).

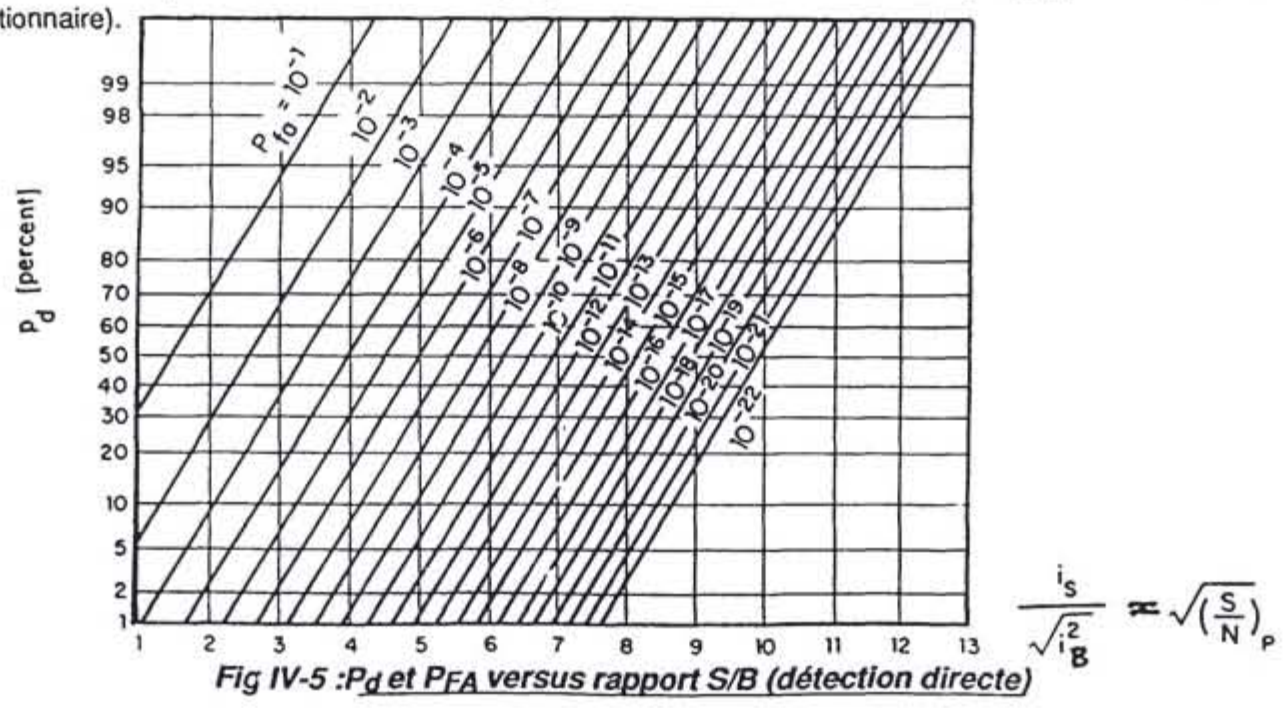




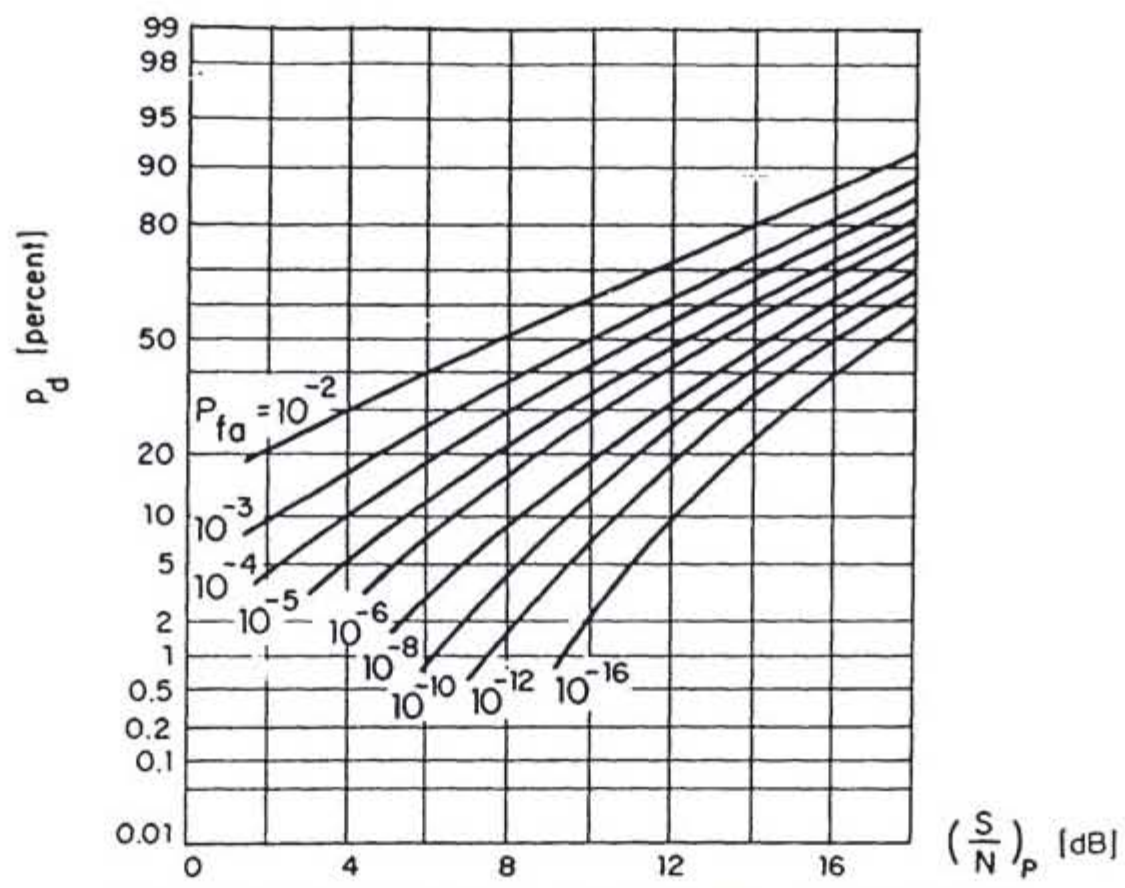

Fig IV-6 : Pd et PFA versus rapport S/B (dét.hét., signal fluctuant)

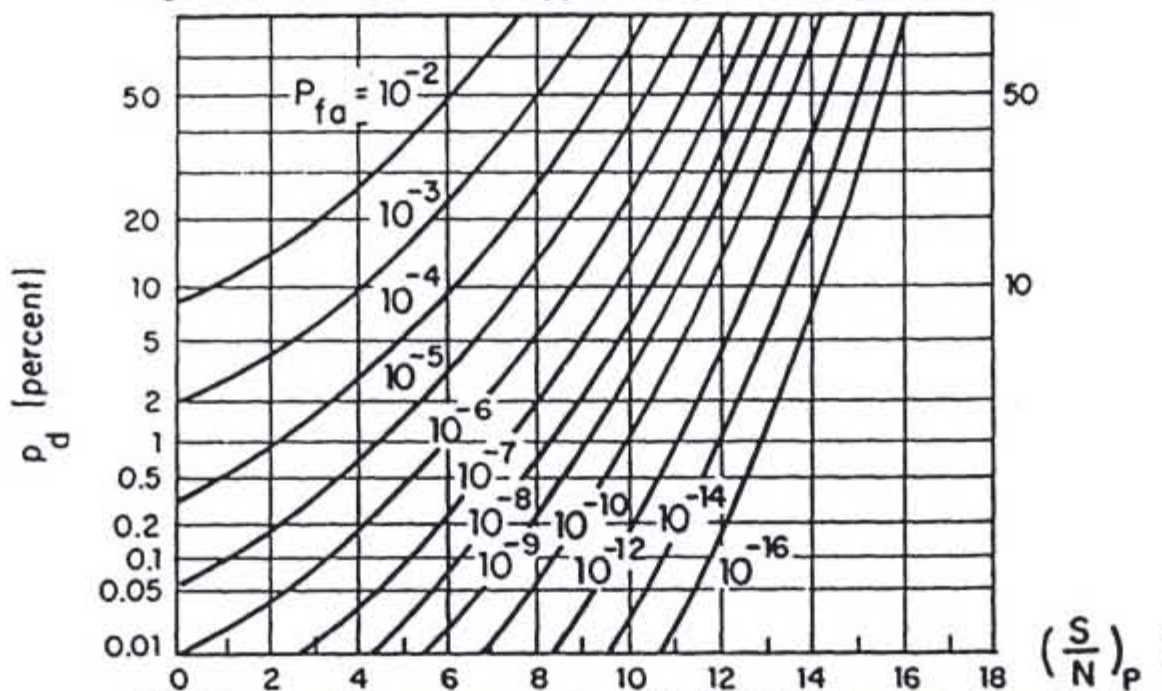

Fig IV-7 : Pd et PFA versus rapport S/B (dét.hét., signal stationnaire)

II faut remarquer que les performances ci-dessus se déduisent du rapport signal à bruit sur une mesure individuelle du phénomène ou signal observé. On rappelle que, si un capteur effectue plusieurs mesures d'un signal donné, sa performance peut s'en trouver améliorée par diverses techniques, dites de "postintégration", dans lesquelles le signal est sommé à lui-même. On peut montrer qu'après sommation de $n$ échantillons de mesure, le rapport signal à bruit vidéo sur la somme est amélioré approximativement de $\sqrt{n}$ vis-â-vis de sa valeur moyenne sur chacune des mesures individuelles 


\section{IV.3.4. EXEMPLE : CALCUL DE LA PORTÉE D'UN TÉLÉMĖTRE LASER A DÉTECTION DIRECTE}

En application des résultats des paragraphes ci-dessus, on propose de calculer la portée d'un télémètre laser à impulsions et détection directe sur un objet plan lambertien de facteur de réflexion diffuse $\mathrm{Rd}$, et perpendiculaire aux rayons. La portée $D_{\max }$ du télémètre sera la distance de l'objet pour laquelle la probabilité de détection est égale à une valeur limite imposée (soit $P_{d}=0,9$ ) pour une probabilité de fausse alarme donnée (soit PFA $=10^{-10}$ )

Le flux laser incident à chaque impulsion sur l'objet situé à une distance $\mathrm{D}$ du télémètre est égal à :

$$
\mathrm{F}_{\mathrm{O}}(\mathrm{D})=\mathrm{F}_{\mathrm{L}} \mathcal{T}_{\mathrm{EM}} \mathcal{T}_{\mathrm{atm}}(\mathrm{D})
$$

où $F_{L}$ est le flux initial de la source laser, $\mathcal{T}_{\text {EM }}$ la transmission de l'optique à l'émission et $\tau_{\text {atm }}(D)$ la transmission atmosphérique à la longueur d'onde du laser sur la distance $D$.

L'intensité en réflexion de l'objet, due au laser est alors :

$$
I_{0}(D)=R_{d} F_{0}(D) / \pi
$$

et le flux laser incident sur le détecteur lors de la réception de l'impulsion est égal à :

$$
\mathrm{Fs}(\mathrm{D})=\tau_{\mathrm{REC}} \tau_{\text {atm(D) }} \text { lo(D) Sop } / \mathrm{D}^{2}
$$

oủ Sop et $\mathcal{T}_{\text {REC }}$ sont la surface de la pupille d'entrée et la transmission de l'optique de réception.

Le rapport (S/B) propre au système, défini à partir du flux équivalent au bruit du détecteur s'écrit en détection directe :

$$
(S / B)=F S(D) / N E P=D^{*}(\lambda) F S(D) / \sqrt{A d} \sqrt{B}
$$

Si limpulsion émise a une durée $\tau$, la bande passante adaptée est approximativement : $B \sim 0,5 / \tau$

La consultation de l'abaque concemant la détection directe montre que ie seuil en rapport signal à bruit permettant d'assurer le couple de valeurs $P_{d}=0,9$ et $P_{F A}=10^{-10}$ est :

$$
(\mathrm{S} / \mathrm{B})_{\min }=7
$$

En résumé, la portée du télémètre est la distance $D_{\max }$ pour laquelle:

$F_{S}\left(D_{\max }\right) / N E P=(S / B)_{\text {min }}$

On rappelle que la transmission atmosphérique sur une distance $\mathrm{D}$ est égale à :

$$
\tau_{\text {atm( }}(D)=\tau_{\text {atmo }}=\exp (\gamma D)
$$

D'où l'expression suivante, qui donne la portée recherchée $D_{\max }$ en fonction des paramètres constitutifs du système :

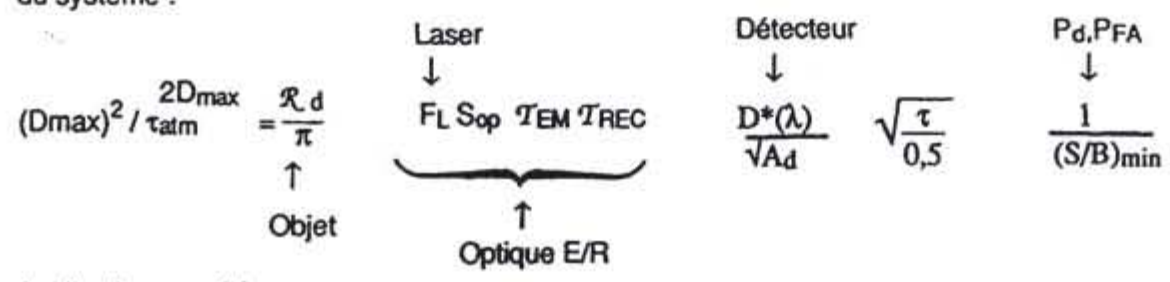

Application n'umérique :

$\left.\begin{array}{ll}F_{L}=10^{6} \mathrm{~W} & \tau=2010^{-9} \mathrm{~s} \\ S_{o p}=10^{-3} \mathrm{~m}^{2} & \tau_{E M}=\tau_{\text {REC }}=0,7 \\ V_{A_{d}}=10^{-2} \mathrm{~cm} & D^{\circ}(\lambda)=10^{11} \mathrm{~cm} \mathrm{~Hz}^{1 / 2} \mathrm{~W}^{-1} \\ R_{d}=0.1 & \tau_{\mathrm{atm}}=0,9\end{array}\right\} \quad D_{\max } \sim 15 \mathrm{~km}$




\section{IV.4. DETECTION PAR OBSERVATION VISUELLE}

Ce paragraphe traite de la détection optique par observation visuelle de scènes, soit en direct (à l'oeil nu). soit au travers d'instruments visuels, généralement destinés à augmenter, par leur grossissement, les dimensions apparentes des objets.

\section{IV.4.1. NOTIONS DE CONTRASTES}

Comme on l'a mentionné dans lintroduction, le cerveau humain appréhende visuellement le monde environnant à partir de la luminance, de la couleur et de la forme des objets. La perception visuelle que l'on a d'un objet présente plusieurs niveaux en fonction de la finesse des détails que l'oeil peut détecter sur cet objet. On distingue ainsi traditionnellement les stades de la détection, simple prise de conscience de la présence de l'objet (dont la nature exacte peut ne pas être définie), de la reconnaissance et de lidentification, qui correspondent à une connaissance de plus en plus précise de l'objet.

Pour illustrer les différences entre ces 3 stades, prenons le cas de l'observation d'un "objet volant" dans le ciel. On aura "détecté" cet objet si l'on remarque la présence d'une petite zone de ciel anormalement plus sombre (ou plus claire) que son environnement. Si l'objet se rapproche, on pourra reconnaitre, c'est-à-dire déterminer à quelle classe il appartient (avion de ligne, avion de combat, hélicoptère, oiseau, etc...). voire l'identifier si l'on peut définir son modèle à l'intérieur de sa classe d'appartenance (par exemple, si l'objet dans le ciel a été reconnu comme étant un hélicoptère. lidentification permet de dire s'il s'agit d'une alouette, d'une gazelle, etc...).

La détection ne suppose aucune connaissance à priori de l'objet, alors que le passage aux stades suivants (reconnaissance et identification) en nécessite une, de plus en plus détaillée (forme, dimensions. éléments caractéristiques).

Si l'on ne tient pas compte de linformation apportée par la couleur (certes importante, mais hors du sujet de cette présentation), la perception d'un objet se fonde essentiellement sur sa forme et les variations relatives de luminance apparente entre zones voisines dans le champ de vision : il peut s'agir de la différence de luminance soit entre l'objet et le fond environnant, soit à l'intérieur de l'objet lui-même.

On traduit ces variations relatives par la notion de "contraste en luminance" qui, selon les auteurs, s'exprime par l'une ou l'autre des 2 relations suivantes:

$$
\begin{gathered}
C_{F}=\left(L_{1}-L_{2}\right) / L_{2} \\
C_{i}=\left(L_{1}-L_{2}\right) /\left(L_{1}+L_{2}\right)
\end{gathered}
$$

où $L_{1}$ et $L_{2}$ sont les luminances des 2 zones contiguës dans l'espace dont on veut mesurer le contraste.

Ces luminances pourront être par exemple respectivement la luminance de robjet (ou $L_{0}$ ) et celle du fond (ou LF). auquel cas on parle du contraste de robjet sur le fond. Elles pourront être aussi bien les luminances de 2 zones situées à lintérieur de la silhouette de lobjet, auquel cas on parlera de contraste interne à l'objet.

Les graphes de la figure IV.8 montrent l'évolution de ces contrastes en fonction de l'une des 2 luminances en jeu, l'autre étant maintenue fixe. On notera que, pour les faibles contrastes $\left(L_{1} \sim L_{2}\right), C_{F}=2 C_{1}$ 


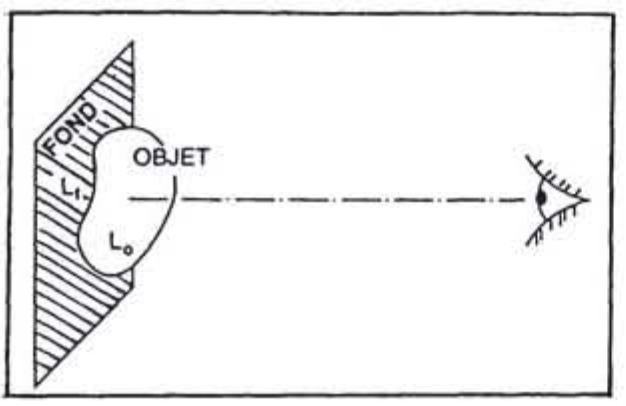

$\mathrm{C}_{\mathrm{F}}$

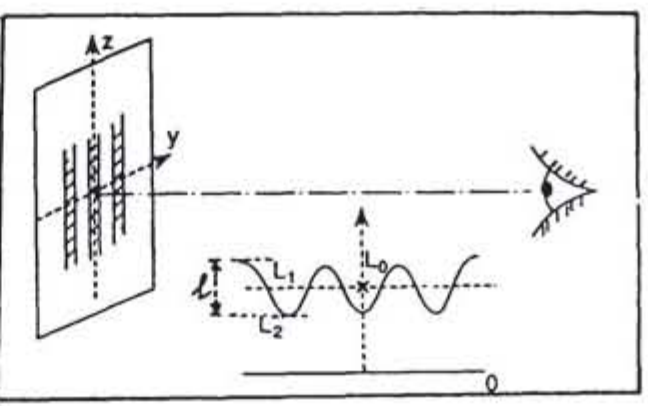

$\mathrm{Ci}_{\mathrm{i}}$

Fig IV-8: Contrastes CFet $C_{i}$

Si l'oeil humain n'est pas très fiable pour la mesure absolue d'une luminance, il est par contre capable de comparer avec une assez bonne précision les luminances de 2 plages contiguës et de déceler de faibles différences entre elles, d'où l'importance de la notion de contraste et du minimum de contraste perceptible dans l'acquisition visuelle d'un objet sur un fond.

II ressort des études sur la sensibilité de l'oeil aux contrastes en luminance que le seuil de contraste minimum perceptible dépend de nombreux facteurs, tels que :

- Dimension de l'objet

- Valeur moyenne des 2 luminances

- Uniformité de la scène

- Durée de l'observation

- État de fatigue du sujet, etc...

La courbe de la figure IV.9 montre la variation du seuil de contraste minimum nécessaire à la détection d'une mire constituée de barres rectilignes, en fonction de la fréquence angulaire de ces demières. exprimée par le rapport entre la distance d'observation et le pas de la mire.

II ressort de cette figure que le contraste minimum perceptible, dans des conditions confortables d'observation est de l'ordre de $2 \%$.

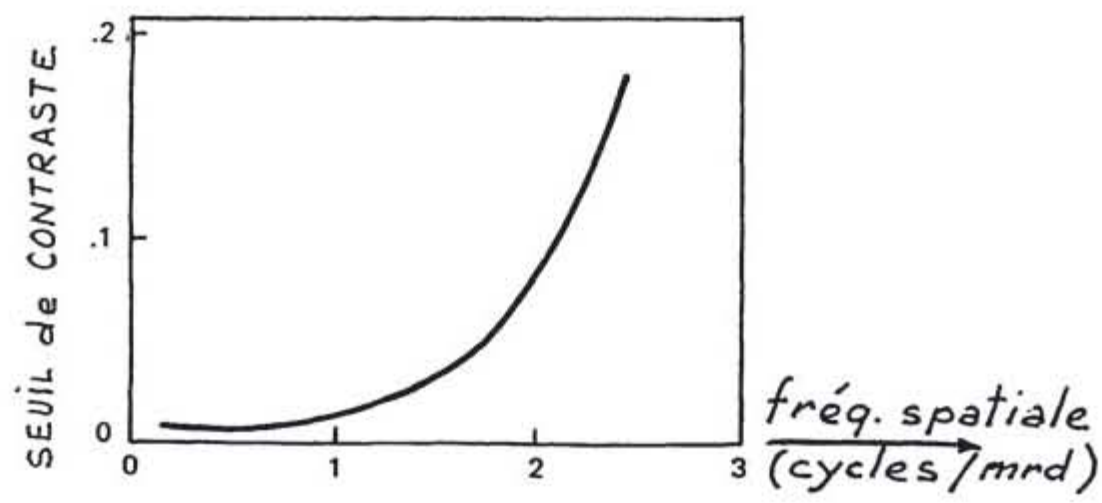

Fig IV-9 : Seull de contraste minimum perceptible 


\section{IV.4.2. DÉTECTION D'UN OBJET SUR FOND D'HORIZON (VISIBILITÉ MÉTÉOROLOGIQUE HORIZONTALE)}

II est bien connu qu'un objet est d'autant plus difficile à détecter dans le visible que la visibilité météorologique est faible. Ce paramètre est donc utile pour apprécier les conditions météorologiques mais aussi parce qu'il permet de définir une distance limite de fonctionnement aux systèmes de perception visuels (oeil nu, jumelles, ....).

Par définition, la visibilité météorologique horizontale est la distance limite de détection visuelle d'un objet noir sur fond d'horizon, c'est-à-dire d'après ce qui a été vu précédemment, la distance $\mathrm{V}$ pour laquelle le contraste apparent de l'objet par rapport à l'horizon qui l'entoure est égal à - $2 \%$.

Un objet noir $\left(L_{0}=0\right)$, observé à courte distance se détache avec un très bon contraste $\left(C_{F}=-1\right)$ sur lhorizon. Si l'observateur s'éloigne de cet objet, latmosphère diffuse la lumière ambiante avec une luminance croissante en fonction de cet éloignement mutuel jusqu'à ce que l'objet devienne indiscemable de lhorizon (contraste nul en luminance).
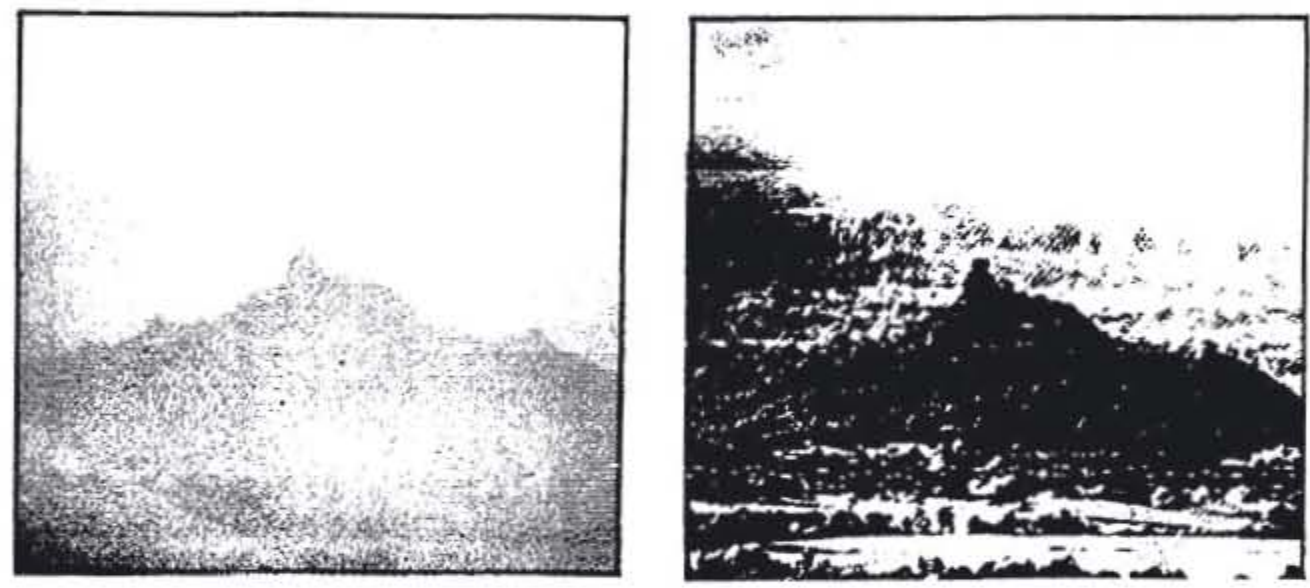

Fig IV-10 : Contraste d'un objet sur fond d'horizon

Si l'on décompose l'atmosphère (situé entre l'objet et l'observateur) en tranches verticales successives perpendiculaires à la direction de visée et d'épaisseur élémentaire dx, on peut montrer (voir paragraphe 1.4.4) que la luminance spectrique en diffusion de chaque "tranche" est liée à son éclairement spectrique par:

$$
\left[\frac{d^{2} L}{d \lambda}\right]_{d i f t}=P(\theta) \frac{\beta(\lambda)}{4 \pi}\left[\frac{d E}{d \lambda}\right] d x
$$

oủ $\mathrm{P}(\theta)$ est la valeur de l'indicatrice de diffusion de latmosphère correspondant à l'angle $\theta$ entre la direction de la source principale drillumination de scène (en général, la direction du soleil) et la direction d'observation et $\beta(\lambda)$ est le coefficient de diffusion spectral linéique de latmosphère, supposé constant pour les trajets horizontaux considérés.

Dans le domaine du visible, la transmission de ratmosphère est essentiellement dictée par la diffusion, de sorte que, sur une distance $x$, elle vaut :

$$
T_{d}(\lambda, x)=\exp [-\beta(\lambda) x]
$$


et la luminance spectrique due à la diffusion de la tranche d'atrmosphère située à la distance $\mathrm{x}$ de l'observateur est égale à :

$$
\left[\frac{d^{2} L}{d \lambda}\right]_{\text {utile }}^{d i f t}=P(\theta) \frac{\beta(\lambda)}{4 \pi}\left[\frac{d E}{d \lambda}\right] e^{-\beta(\lambda) x} d x
$$

On déduit la luminance spectrique apparente de l'objet en intégrant les contributions en diffusion de toutes les "tranches" d'atmosphère situées entre l'objet et l'observateur, soit :

$$
\left[\frac{d L}{d \lambda}\right]_{\text {app.objet }}=\frac{P(\theta)}{4 \pi}\left[\frac{d E}{d \lambda}\right]\left[1-e^{-\beta(\lambda) d}\right]
$$

La luminance spectrique de l'horizon se calcule suivant la même procédure en considérant l'atmosphère homogène sur une très grande distance horizontale (hypothèse dite de la terre "plate") :

$$
\left[\frac{d L}{d \lambda}\right]_{\text {horizon }}=\frac{P(\theta)}{4 \pi}\left[\frac{d E}{d \lambda}\right]
$$

Le contraste apparent en luminance de l'objet noir sur fond d'horizon s'obtient alors de façon simplifiée, en considérant l'oeil humain comme un filtre passe bande (à bande étroite) centré à $\lambda=0,555 \mu \mathrm{m}$ (en vision photopique), soit :

$$
\begin{aligned}
C_{F}(d) & =\left(L_{\text {obj }}(d)-L_{\text {horizon }}\right) / L_{\text {Horizon }} \\
= & \frac{\int_{0}^{\infty} K m V(\lambda)\left[\frac{d L}{d \lambda}\right]_{o b j}^{d i f} d \lambda-\int_{0}^{\infty} K m V(\lambda)\left[\frac{d L}{d \lambda}\right]_{\text {Hor }}^{d i f} d \lambda}{\int_{0}^{\infty} k_{m} V(\lambda)\left[\frac{d L}{d \lambda}\right]_{\text {Hor }}^{d i f} d \lambda} \\
& =\exp [-\beta(\lambda) d] \& \lambda=0,555 \mu m
\end{aligned}
$$

Par définition, lorsque l'objet se trouve à la distance de visibilité météorologique horizontale, il est en limite de détection et son contraste apparent sur fond d'horizon est égal à $-2 \%$ :

$$
C_{F}(V)=-2 \%=-\exp [-\beta(0,555) V]
$$

d'où la relation entre la distance de visibilité météorologique horizontale et le coefficient de diffusion linéique de l'atmosphère $(\mathrm{a} \lambda=0,555 \mu \mathrm{m})$ :

$$
V=3,91 / \beta(0,555 \mu \mathrm{m})
$$

\section{IV.5. DETECTION PAR VISUALISATION SUR ECRAN}

De plus en plus nombreux sont les systèmes optiques ("capteurs dimages") qui forment limage d'une scène et la présentent ensuite à un observateur sur un écran de visualisation. Les exemples les plus représentatifs de tels systèmes optiques sont les caméras de télévision, camescopes, etc... qui, fonctionnant dans le visible, restituent de la scène une imagerie assez proche (à la résolution près) de celle que verrait l'observateur en direct. II apparait aussi de nouveaux capteurs d'images pour observation dans les "fenêtres" infrarouges de l'atmosphère, dont les applicaricns sont nombreuses dans la défense et le spatial, et pour lesquels une transposition đrimage dans le visible s'avère évidemment nécessaire pour qu'une observation humaine en soit possible. 


\section{IV.5.1. RAPPELS GÉNÉraUX SUR LA VISUALISATION DE TYPE TÉLÉVISION}

Dans le standard le plus utilisé en visualisation (ou standard CCIR de télévision), le champ de vision du capteur est balayé suivant un ensemble de lignes horizontales. Chaque image est composée de 2 trames entrelacées, une trame regroupant les lignes paires et l'autre les lignes impaires, pour réduire l'effet de papillotement (ou flicker), phénomène ressenti par l'observateur lorsque les images lui sont présentées à cadence faible. Les fréquences de répétition des trames et des images sont respectivement de 50 et 25 $\mathrm{Hz}$ (60 et $30 \mathrm{~Hz}$ aux USA). Chaque image comporte 575 lignes utiles avec, pour l'instant, un format (rapport entre la largeur et la hauteur) de 4/3. La distance d'observation qui est recommandée pour adapter la dimension angulaire d'un point élémentaire de limage sur le moniteur à la résolution moyenne de l'oeil $\left(\sim 3.10^{-4} \mathrm{rd}\right)$ est de l'ordre de 5 fois la diagonale de l'écran.

Suivant les résultats du paragraphe 1.3 .4 , un capteur d'image est sensible à la luminance apparente de la scène pour chaque direction du champ observé. A partir de la luminance spectrique initiale de chaque zone de la scène (objet ou fond) :

$$
\left[\frac{d L}{d \lambda}\right]=\varepsilon(\lambda, T)\left[\frac{d L}{d \lambda}\right]_{C N}^{T}+\frac{R d}{\pi}\left[\frac{d E}{d \lambda}\right]
$$

On déduit pour cette direction l'expression de la luminance spectrique apparente en entrée de pupille :

puis l'éclairement spectrique $\left[\frac{d E^{\prime}}{d \lambda}\right]$ du détecteur concerné :

$$
\frac{d L_{a p \rho}}{d \lambda}=\tau_{\text {atm }}(\lambda)\left[\frac{d L}{d \lambda}\right]+\frac{d L p}{d \lambda}+\frac{d L d}{d \lambda}
$$

$$
\left[\frac{d E^{\prime}}{d \lambda}\right]=\frac{\pi \mathcal{L}_{o p}(\lambda)}{4 N^{2}}\left[\frac{d L_{a p p}}{d \lambda}\right]
$$

Suivant la conception du capteur utilisé, chaque détecteur répond, pour la direction de l'espace qui lui est impartie, avec un signal proportionnel soit à son éclairement instantané soit à son exposition sur la durée $\tau$ d'une image $(\tau=40 \mathrm{~ms})$, d'où les expressions suivantes de signal :

$$
\begin{aligned}
i_{s} & =A_{d} \int_{0}^{\infty} R_{i}(\lambda)\left(\frac{d E^{\prime}}{d \lambda}\right) d \lambda \\
\text { où } q_{s} & =A_{d} \int_{t}^{H \pi} \int_{0}^{\infty} R_{i}(\lambda)\left(\frac{d E^{\prime}}{d \lambda}\right) d \lambda
\end{aligned}
$$

Ces valeurs sont représentatives de chacun des pixels d'une image et l'électronique du capteur les remet en forme dans le temps, de façon conforme au balayage ligne d'une image TV. Le signal temporel résultant dit "signal vidéo" est transmis à un moniteur qui le traduit alors en spot lumineux dont le balayage et les variations de luminance constituent la base de l'imagerie sur écran.

Dans la plupart des applications d'imagerie (exception faite de la métrologie), la composante continue des signaux issus des détecteurs au cours du balayage est éliminée par liaison capacitive au sein du capteur. de sorte que le signal vidéo en sortie n'est représentatif que des variations de luminance apparente de la scẻne le long d'une ligne (réelle ou fictive dans le cas où les facteurs de réponse des détecteurs ne sont pas identiques).

C'est donc à lobservateur de restituer, en fonction de ses préférences en confort visuel, la luminance moyenne de l'image sur le moniteur, grâce à un réglage dit "de luminosité". II peut aussi ajuster le contraste de limage en réglant, de façon indépendante au réglage précédent, le "gain" du moniteur, qui lie les variations en luminance du spot à celles du signal vidéo. 
Ainsi, pour un contraste apparent de scène donné, c'est-à-dire pour un ensemble : objet - fond atmosphère - capteur, imposé, le contraste de limage visuelle (défini au paragraphe IV.4.1) sur le moniteur peut prendre des valeurs très diverses, en fonction des préférences en observation de lutilisateur.

IV.5.2. NOTION DE RAPPORT SIGNAL A BRUIT PERÇU

Vis-à-vis de l'observation visuelle d'une scène en direct ou par instrument visuel, limagerie de la même scène par un système à visualisation présente des différences fondamentales : en plus du domaine spectral et de la résolution angulaire (qui peuvent être très proches ou éloignés l'un de l'autre), on notera les 2 points particuliers suivants, qui caractérisent l'imagerie sur moniteur :

- D'une part, la possibilité de modification du contraste par l'observateur (réglages indépendants en "luminosité" et "contraste") (voir paragraphe IV.5.1)

- D'autre part, la présence de bruit en luminance, de pixel en pixel, provenant des fluctuations de signal du détecteur (voir paragraphe III.3).

Ces particularités ont conduit à la mise sur pied de procédures spécifiques à l'évaluation des performances en détection des systèmes optiques à visualisation, qui ne s'appuient pas sur la notion de contraste (propre aux systèmes visuels), mais plutôt sur celle de rapport signal à bruit dans l'image.

Dans ces procédures, on considère l'image sur l'écran comme un signal spatio-temporel (bruité) vis-à-vis duquel l'oeil et le cerveau de l'observateur se comportent de façon similaire à un détecteur à seuil : l'observateur d'une image sur moniteur ne pourra détecter, reconnaître ou identifier un objet vis-à-vis d'un fond donné, que s'il perçoit la présence de cet objet dans l'image avec un rapport signal à bruit (dénommé "rapport S/B perçu") supérieur à un seuil, dont la valeur a été déterminée par mesures statistiques sur un grand nombre d'expérimentateurs.

Pour définir cette notion de rapport S/B perçu par l'observateur, considérons le cas simple de l'observation sur moniteur d'un objet rectangulaire (appelé "mire") de luminance uniforme Lo, à détecter sur un fond de luminance uniforme $L$. Lors du balayage de la scène par le capteur optique, le signal vidéo (par exemple en tension) passe d'une valeur moyenne $v_{0}$ sur l'objet à une valeur $v_{f}$ sur le fond. Le rapport signal à bruit vidéo en sortie de capteur est par définition égal à :

$(S / B)_{v i d s o}=\left|v_{0}-v_{f}\right| / v_{b}$

où $\mathrm{vb}$ est la tension efficace de bruit (voir figure IV-11)

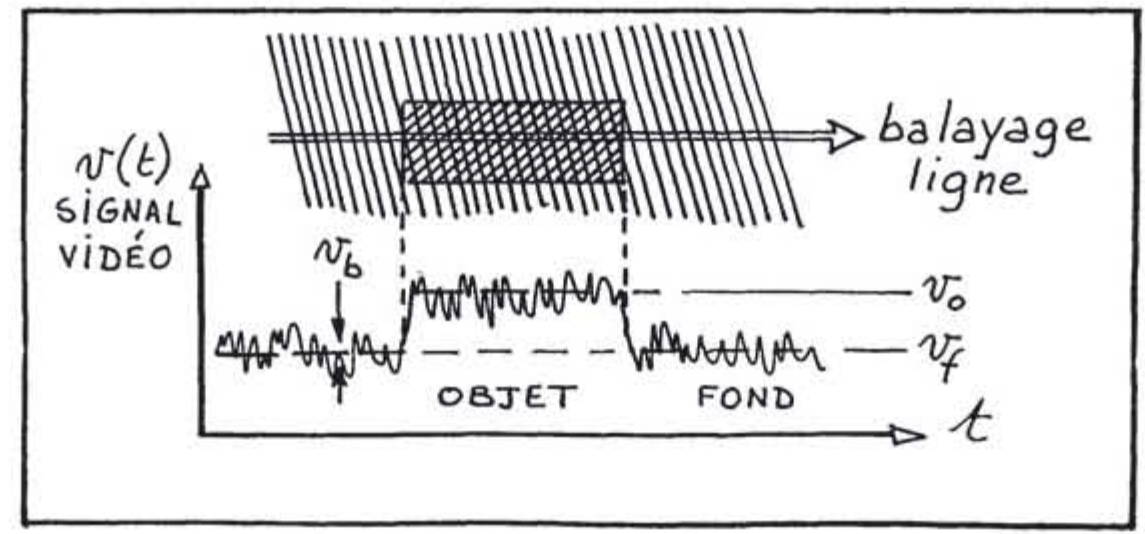

Figure IV-11 : Rapport signal à bruit vidéo 
Dans un angle solide réduit (diamètre inférieur à $0,5^{\circ}$ environ), situé au centre du champ de vision (fovéa). l'oeil et le cerveau humain intègrent spatialement et temporellement les éléments de résolution de l'image par zones de luminance uniforme. Cette postintégration locale aboutit à l'amélioration du rapport signal à bruit (voir fin du paragraphe IV.3.3) qui, au niveau de l'observateur, prend la valeur $(S / B)_{p}$ suivante :

$$
(\mathrm{S} / \mathrm{B})_{p}=(\mathrm{S} / \mathrm{B})_{\mathrm{vidé}} \sqrt{N_{S}} \sqrt{N_{T}}
$$

oủ Ns Nombre d'éléments de résolution sur la zone de luminance homogène définissant l'objet.

NT Nombre d'images successivement présentées à l'observateur pendant la durée d'intégration de l'oeil ( 0,2 s). Dans le standard CCIR européen (25 images/s), NT = 5.

Les expérimentations menées sur un grand nombre d'observateurs pour déterminer leur capacité à détecter un objet sur fond uniforme ont conduit à l'établissement d'une courbe standard (Fig IV-12) donnant la probabilité de détection (dans le cas de mires rectangulaires) en fonction du rapport signal à bruit perçu.

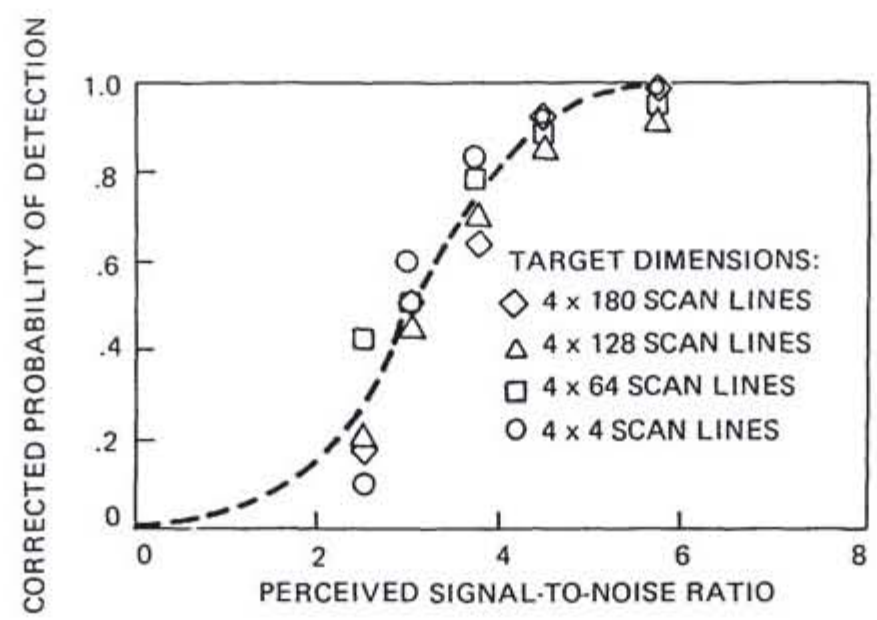

Figure IV-12: Probabilité de détection en fonction du rapport S/B perçu (objets rectangulaires)

\section{IV.5.3. PROCÉDURE GÉNÉRALE D'ÉVALUATION DE SYSTÉMES OPTIQUES A VISUALISATION}

La performance d'un système optique à visualisation s'évalue par les capacités qu'il offre à l'observateur d'appréhender la présence et/ou la nature de l'objet recherché sur le moniteur. Elle dépend en général d'un grand nombre de paramètres, tels que les caractéristiques géométriques et photométriques de l'objet et du fond, le domaine spectral de fonctionnement du système et les propriétés optiques de latmosphère qui en découlent. D'après le paragraphe précédent, l'observateur pourra appréhender l'objet si le rapport signal à bruit qu'il en perçoit est supérieur à un seuil donné, soit sur lensemble de cet objet lorsqu'il est angulairement petit (phase de détection), soit sur des détails plus ou moins fins (phases de reconnaissance et d'identification).

Une procédure (ou modélisation) souvent utilisée pour évaluer la performance théorique de tels systèmes optiques dans des conditions données (objet, fond, atmosphère) consiste à remplacer l'objet par une mire dite "équivalente", à calculer le rapport signal à bruit correspondant perçu par l'observateur et à déduire la probabilité de détection d'après la courbe de la Figure IV-12. 
Les caractéristiques géométriques et photométriques de la mire "équivalente à l'objet" dépendent à la fois de l'objet recherché, du fond sur lequel il est supposé apparaitre et de la mission confiée à lobservateur : la mire sera différente s'il s'agit de détection, de reconnaissance ou d'identification d'objet.

Ces caractéristiques, définies par un ensemble de critères, ont été établies à l'issue de nombreuses expérimentations sur la détection d'objets et de leurs mires équivalentes. Les critères les plus courants sont les "critères de Johnson", selon lesquels la mire équivalente à un objet est définie de la façon suivante :

- Elle est rectangulaire (largeur I, longueur L), circonscrite à l'objet lui-même. Elle comporte des barres disposées parallèlement à sa longueur et dont les luminances sont alternativement celles de l'objet et du fond avoisinant.

- Le nombre de paires de barres successives le long de sa largeur est dicté par le type de mission envisagé :

- 2 paires en détection

- 4 paires en reconnaissance

- 7 paires en identification

La figure IV-13 illustre cette notion de "mire équivalente".

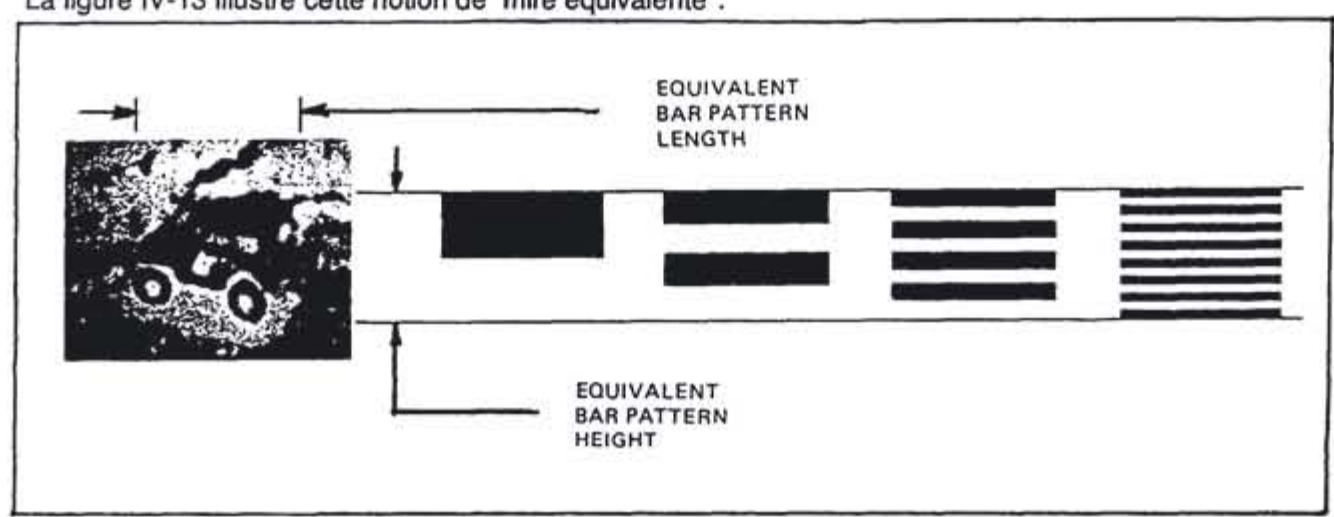

Fig IV-13 : Notion de "mire équivalente" à un objet sur fond donné

Les principales étapes à suivre pour évaluer les performances d'un système optique à visualisation suivant cette procédure, par exemple, pour calculer la portée d'un tel système (ou distance au delà de laquelle le système n'assure plus sa mission avec la probabilité de réussite exigée), sont résumées ci-dessous :

- Calcul des propriétés photométriques (dans la plupart des cas, luminances spectriques) de l'objet et du fond environnant. Ce calcul s'effectue d'abord au niveau de l'objet, puis à celui du capteur d'après la piocédure du paragraphe II.1.4

- Définition de la mire équivalente à robjet.

- Dimensions : largeur I x longueur L

- Nombre de paires de barres sur la largeur, d'où la fréquence spatiale de la mire

- Attribution des luminances dobjet et de fond aux barres respectives

- Evaluation du signal vidéo en sortie de capteur correspondant au passage du système de balayage sur les zones de mire représentatives respectivement de l'objet et du fond. Ne pas oublier de tenir compte de la fonction de transfert de modulation de l'ensemble du capteur (optique, détecteur. électronique). 
- Calcul du bruit du système au point de mesure défini ci-dessus (d'après le paragraphe III.3)

- Calcul du rapport signal à bruit vidéo $(S / B)_{v}$ délivré par le système au niveau du moniteur. Pour cela, on prendra en compte la dégradation de signal due au moniteur, à la fréquence définie par le pas de la mire

- Calcul du rapport ( $\mathrm{S} / \mathrm{B})_{p}$ perçu par l'observateur lorsqu'il regarde la mire sur le moniteur. L'expression de ce rapport (S/B)p se déduit des résultats du paragrapphe IV.5.2 ci-dessus en y intégrant la fonction de transfert de modulation de l'oeil (FTM(oeil), à la fréquence angulaire définie par l'image de la mire et la distance d'observation de l'oeil au moniteur, pour aboutir à :

$$
(\mathrm{S} / \mathrm{B})_{\mathrm{p}}=\mathrm{FTM} \text { (oeil) } \times(\mathrm{S} / \mathrm{B})_{\mathrm{v}} \times \sqrt{5 \mathrm{NS}_{\mathrm{S}}}
$$

où Ns est le nombre d'éléments de résolution de l'oeil par barre de la mire.

Toutes ces étapes sont schématisées sur le tableau IV-2 ci-dessous :

Tableau IV-2 : Procédure générale d'évaluation d'un système optique à visualisation

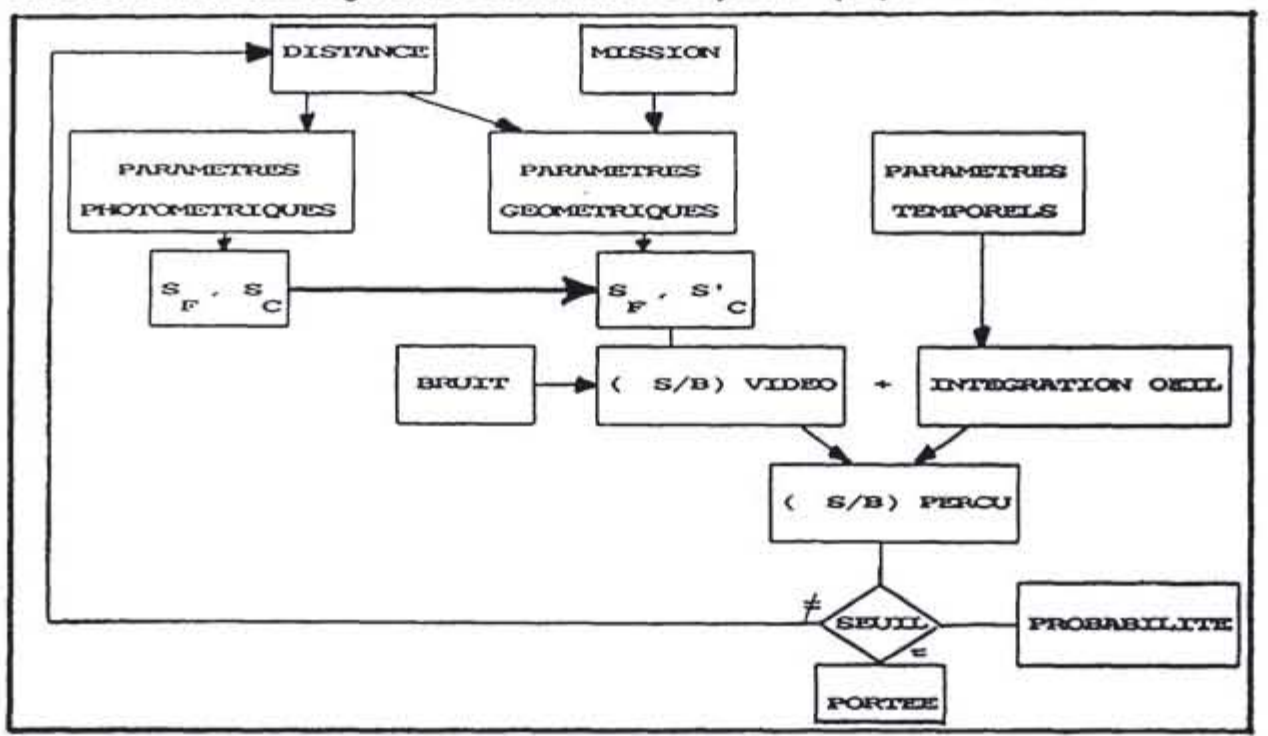

IV.5.4. EXEMPLE D'APPLICATION : IMAGERIE INFRAROUGE

L'imagerie infrarouge (ou thermique) extrait d'une scène les informations provenant de la différence de température apparente entre zones et les transpose sous forme d'images dans le visible. La performance de ces capteurs (caméras infrarouges ou FLIR) est évaluée en fonction de leur aptitude à faire ressortir la présence de petits objets dont la température est proche de celle du fond en ironnant. On demande donc à ces capteurs à la fois une bonne sensibilité thermique et une bonne résolution angulaire.

La sensibilité thermique d'un système d'imagerie infrarouge s'évalue grâce au calcul du rapport signal à bruit vidéo délivré par le capteur lorsqu'on observe la ligne de démarcation entre 2 Corps Noirs étendus et portés à 2 températures apparentes proches l'une de l'autre, $T$ et $T+\Delta T$. On peut démontrer que le signal vidéo obtenu lors du passage du système de balayage d'un Corps Noir à l'autre est proportionnel à cette différence de températures apparentes : $v S=K \Delta T$ et définir ainsi une nction, similaire à celle du flux équivalent au bruit ou NEP (paragraphe III.3), dénommée NETD ou différence de température équivalente au bruit, égale à la différence de température apparente entre corps noirs qui entraine un rapport signal à bruit vidéo de valeur égale à 1, d’où : 


$$
\frac{V_{s}}{V_{b}}=(S / B)_{\text {video }}=\frac{D^{*} \Delta F S}{\sqrt{A_{d}} \sqrt{B}}=\Delta T / N E I D
$$

expression dans laquelle $\Delta \mathrm{Fs}$ représente la variation du flux de scène reçu par le détecteur au cours du passage du système de balayage d'un Corps Noir à lautre, $D^{*}$ la détectivité spécifique moyenne du détecteur sur la bande spectrale de fonctionnement. Ad Paire du détecteur et $\mathrm{B}$ la bande passante électronique du système.

La formulation ci-dessous très simplifiée du NETD fait ressortir les principaux paramètres qui définissent sa valeur :

$$
N E T D=\frac{\sqrt{A_{\alpha}} \sqrt{B}}{\Omega A_{o \rho} D^{*} T_{\alpha \rho}\left({ }^{2} / \partial T\right)}
$$

où $\Omega$ est le champ de réception vu par chaque détecteur, Aop et Top l'aire et la transmission de l'optique de réception, et $\partial \mathrm{U}$ गT la loi de variation en luminance du Corps Noir en fonction de sa température, pour la bande spectrale de fonctionnement considérée.

La figure IV-14 illustre cette loi de variation ( $\partial \mathrm{L} / \mathrm{T}$ ) dans les 2 bandes spectrales les plus usuelles de limagerie infrarouge, c'est-à-dire $3 / 5 \mu \mathrm{m}$ et $8 / 12 \mu \mathrm{m}$ et donne le rapport correspondant pour des températures comprises entre 240 et $320 \mathrm{~K}$.

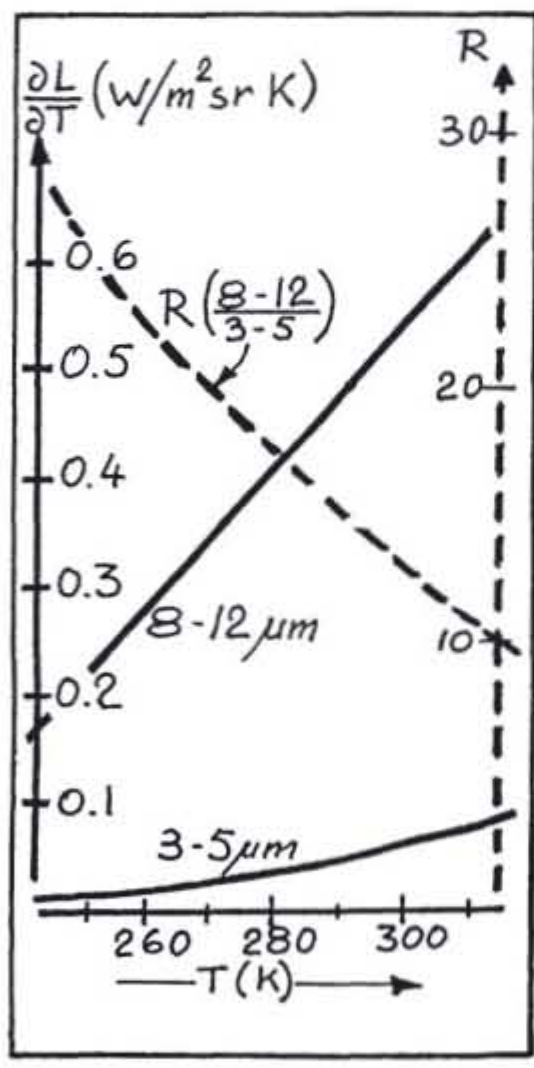

Figure IV-14

Variation de la luminance du $\mathrm{CN}$ en fonction de $\mathrm{T}$
II apparait qu'aux températures ambiantes ou proches de lambiante, la luminance du Corps Noir varie beaucoup plus en fonction de la température dans la bande $8 / 12 \mu \mathrm{m}$ que dans la bande $3 / 5 \mu \mathrm{m}$ ce qui entraine théoriquement une meilleure sensibilité thermique des systèmes infrarouges dans la bande $8 / 12 \mu \mathrm{m}$ (les autres paramètres étant supposés identiques).

Cependant, le choix de la bande spectrale d'une caméra irfrarouge en vue de l'optimisation de ses performances repose sur un nombre important de paramètres, en particulier, la qualité des détecteurs (nombre, uniformitè. rendement quantique) les propriétés spectrales de latmosphère, la résolution angulaire du système, etc...

Bien que très utile, la notion de NETD ne suffit pas pour décrire complètement les performances d'un système d'imagerie infrarouge, car elle ne fait pas intervenir la qualité optique du système (résolution angulaire). De deux instruments identiques en NETD, le plus performant sera évidemment celui qui a la meilleure résolution, car it conduira à une reconnaissance et une identification plus rapides de l'objet.

Pour caractériser de façon plus globale un système d'imagerie infrarouge, c'est-à-dire pour prendre en compte à la fois sa sensibilité thermique et sa résolution angulaire. on utilise 'a notion de Différence de Température Minimale Perceptible (ou MRTD : Minimum Resolvable Temperature Difference), définie ci-après. Alors que la NETD est la difference de température apparente entre 2 corps Noirs étendus conduisant à un rapport (S/B) vidéo égal à 1, la 
MRTD est la différence de température apparente entre les barres d'une mire thermique correspondant à la limite de perception de œs barres par un observateur lorsque $œ$ dernier observe l'image sur le moniteur.

La notion de MRTD est beaucoup plus complète que celle de NETD puisqu'elle intègre les paramètres géométriques de la mire et donc de l'objet (pas de la mire, fréquence spatiale ou angulaire) et qu'elle est définie par la détection de l'objet au niveau de l'observateur.

Pour l'évaluation approximative de la MRTD d'un système IR, considérons que ce dernier vise une mire thermique dont les barres sont aux températures apparentes $T$ et $T+\Delta T$. Les mires thermiques pour mesure de MRTD ont une forme normalisée : elles sont carrées et comportent 7 barres, de sorte que le facteur de forme de chaque barre (rapport entre la largeur et la longueur) est de $1 / 7$.

Pour une différence de température $\Delta T$ entre barres, le rapport signal à bruit perçu par l'observateur sur l'image de la mire au moniteur est égal à :

$$
(S / B)_{p}=\sqrt{5 N_{S}} \text { FTMoeil FTMmoniteur } \quad(S / B) \text { vidéo }
$$

où (S/B) vidéo,mire est le rapport signal à bruit vidéo donné par le capteur sur les barres de la mire et Ns le nombre d'éléments de résolution du système sur une barre de la mire. II vient alors :

$$
\left(S / B_{p}=\sqrt{5 N_{S}} F T M g \Delta T / N E T D\right.
$$

où FTMg est la FTM globale du système (observateur compris) :

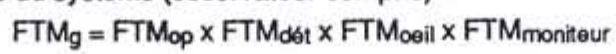

Par définition, la MRTD du système infrarouge est la différence de température minimale entre barres de la mire qui permette à l'observateur de détecter leur présence, avec une probabilité imposée donc qui entraîne une valeur de rapport signal à bruit perçu supérieure ou égale à un seuil ks, d'où :

$$
M T R D=\frac{k S N E T D}{F T M g \sqrt{5 N S}}
$$

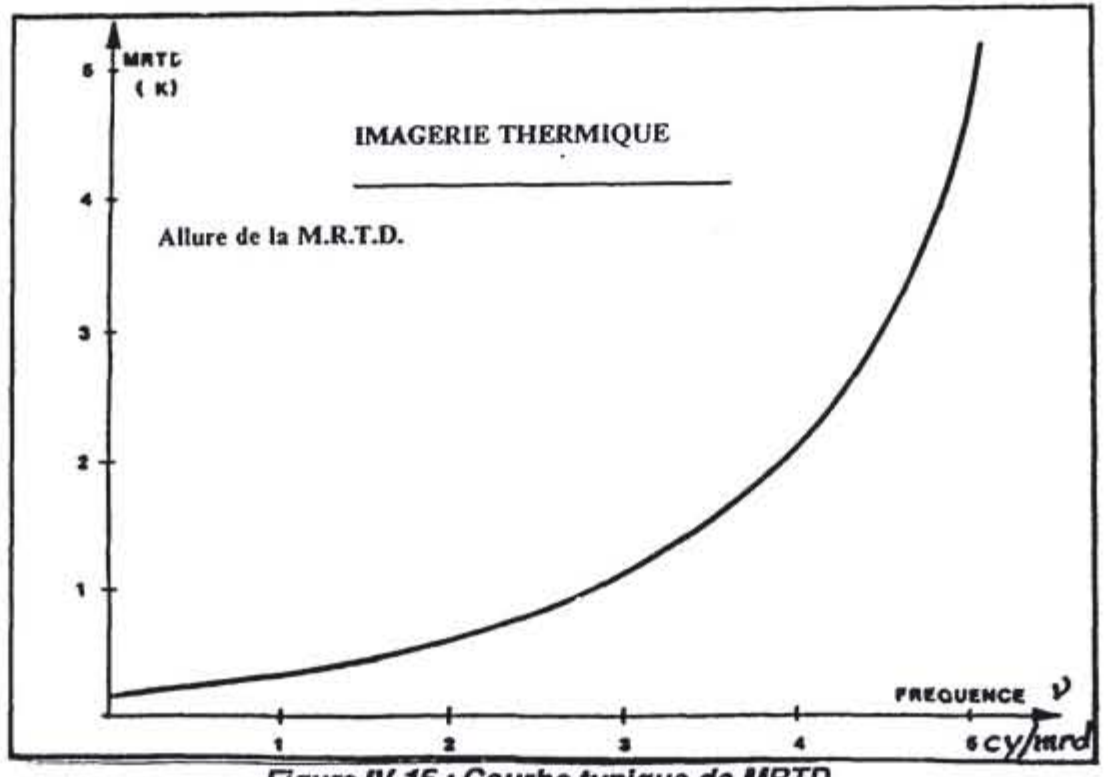

Figure IV-15: Courbe typique de MRTD 
Par exemple, si la probabilité de détection imposée est de $90 \%$, la figure IV-11 montre que la valeur de ks correspondante est : $k s=4,5$.

La valeur de la MRTD d'une caméra thermique dépend de la fréquence spatiale de la mire par lintermédiaire de la fonction de transfert globale FTMg et du nombre Ns d'éléments de résolution du système dans chaque barre de la mire. On caractérise donc généralement une caméra thermique par la courbe donnant révolution de la MRTD en fonction de la tréquence spatiale angulaire $f_{M}$ de la mire utilisée (en cycles/mrd), dont la figure IV-15 donne lallure typique :

A partir de cette courbe de MRTD, on peut déduire assez simplement la portée d'un système d'imagerie infrarouge. La notion de MRTD conduit assez naturellement à l'évaluation des performances du système de reconnaissance, car la configuration de la mire de MRTD est assez proche de celle définie par Johnson pour les tâches de reconnaissance (I'une possède 7 barres alors que l'autre en possède 8 ).

A partir des dimensions de l'objet recherché, on convertit le type de courbe ci-dessus (MRTD en fonction de la fréquence spatiale de la mire) en une variation de MRTD en fonction de la distance de l'objet, en notant que la fréquence spatiale angulaire de la mire équivalente à un objet de largeur l et à une distance D est égale à :

$$
f_{M}=\frac{7}{l} D
$$

D'autre part, la différence de température apparente $\Delta \mathrm{T}_{\mathrm{a}}$ de cet objet, pour une différence de température initiale vis-à-vis du fond égale à $\Delta T \mathrm{~T}$, est égale à :

$$
\Delta T_{a}(D)=\Delta T_{i} \times T_{a t m}(D)
$$

Graphiquement, la portée d'un système d'imagerie IR en reconnaissance s'obtient à l'intersection des 2 courbes MRTD(D) et $\Delta T_{a}(D)$, comme l'indique le graphe de la figure IV-16 ci-dessous :

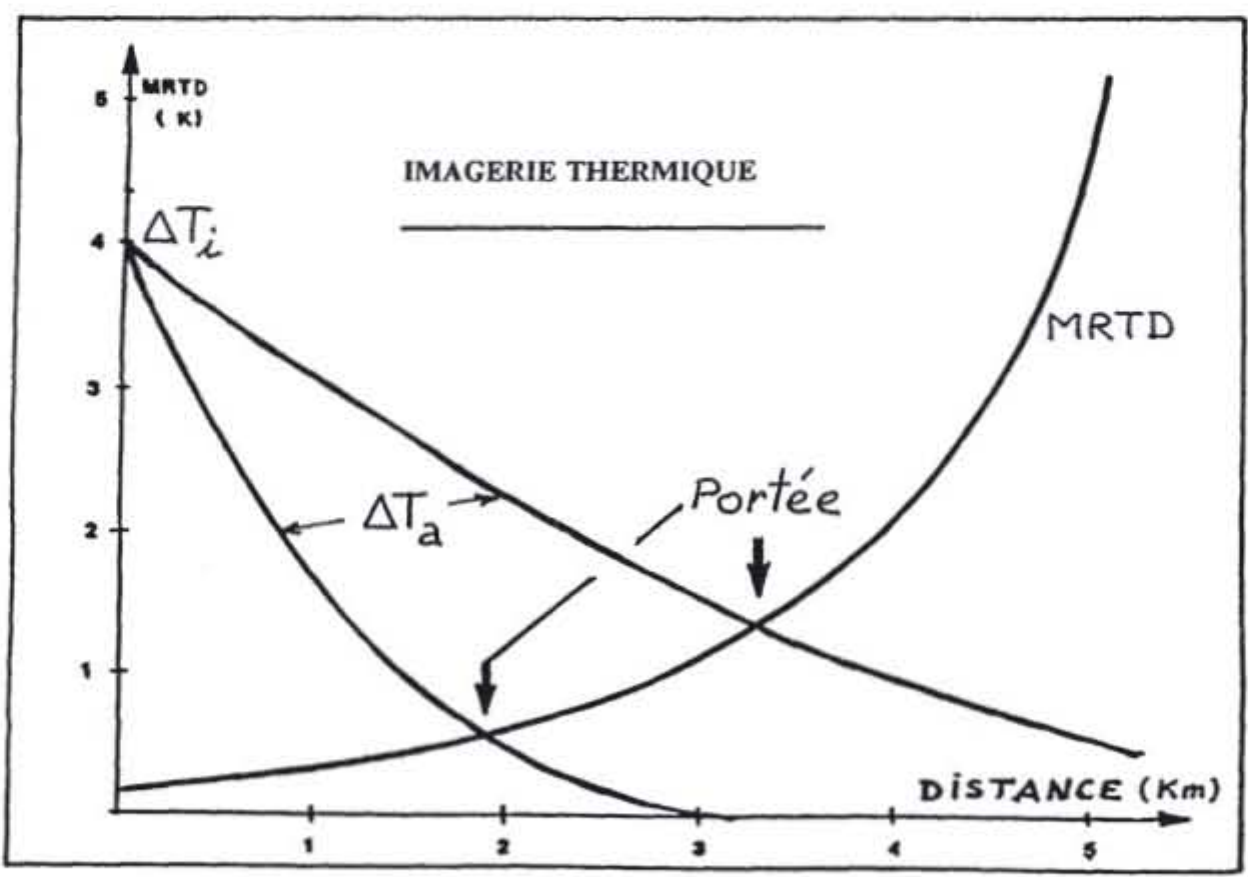

Figure IV-16 : Portée d'un système d'imagerie 


\section{REFERENCES}

1) La Thermographie infrarouge

G.Gaussorgues

Technique et Documentation (Lavoisier), 1981

2) Détection et détecteurs de rayonnements optiques

F.Desvignes

Masson, 1987

3) Rayonnements optiques, Radiométrie - Photométrie

F.Desvignes

Masson, 1991

4) The Infra Red Handbook

W.L.Wolfe et G.J.Zissis, éditeurs

Office of Naval Research, Dpt of the Navy

(disponible chez Lavoisier, 11 rue Lavoisier, Paris, $8^{\circ}$ )

5) Optical Radiation Measurements, Radiometry

F.Grum, R.J.Becherer

Academic Press, 1979

6) Radiometry and the Detection of Optical Radiation

R.W.Boyd

Wiley, 1983

7) Detection of Optical and Infrared Radiation

R.H.Kingston

Springer Verlag, 1978

8) Photometry and Radiometry for Engineers

A.Stimson

Wiley, 1974

9) Thermal Imaging Systems

J.M. Lloyd

Plenum Press, 1975

10) Normes de sécurité laser:

Norme OTAN : "STANAG 3606"

Norme Britannique : "BS 7192:1989"

11) Safety with lasers and other optical sources D.Sliney and M.Wolbarsht

Plenum Press, 1980 